\title{
Teamwork of clinical teachers in postgraduate medical training
}

Citation for published version (APA):

Slootweg, I. A. (2015). Teamwork of clinical teachers in postgraduate medical training. [Doctoral Thesis, Maastricht University]. Maastricht University. https://doi.org/10.26481/dis.20150619is

Document status and date:

Published: 01/01/2015

DOI:

$10.26481 /$ dis.20150619is

Document Version:

Publisher's PDF, also known as Version of record

\section{Please check the document version of this publication:}

- A submitted manuscript is the version of the article upon submission and before peer-review. There can be important differences between the submitted version and the official published version of record.

People interested in the research are advised to contact the author for the final version of the publication, or visit the DOI to the publisher's website.

- The final author version and the galley proof are versions of the publication after peer review.

- The final published version features the final layout of the paper including the volume, issue and page numbers.

Link to publication

\footnotetext{
General rights rights.

- You may freely distribute the URL identifying the publication in the public portal. please follow below link for the End User Agreement:

www.umlib.nl/taverne-license

Take down policy

If you believe that this document breaches copyright please contact us at:

repository@maastrichtuniversity.nl

providing details and we will investigate your claim.
}

Copyright and moral rights for the publications made accessible in the public portal are retained by the authors and/or other copyright owners and it is a condition of accessing publications that users recognise and abide by the legal requirements associated with these

- Users may download and print one copy of any publication from the public portal for the purpose of private study or research.

- You may not further distribute the material or use it for any profit-making activity or commercial gain

If the publication is distributed under the terms of Article $25 \mathrm{fa}$ of the Dutch Copyright Act, indicated by the "Taverne" license above, 


\section{Teamwork of Clinical Teachers in Postgraduate Medical Training}




\section{Teamwork of Clinical Teachers in}

\section{Postgraduate Medical Training}

Irene Slootweg 
ISBN: 978-94-6233-003-0

The copyright of articles that have been published has been transferred to the respective journals.

The research is part of the research project 'Quality of Clinical Teachers and Residency Training Programs' which is co-financed by the Dutch Ministry of Health; the Faculty of Health and Life Sciences of the University of Maastricht; and the Academic Medical Center, Amsterdam.

Printing of this thesis was financially supported by the Professional Performance Research Group, Amsterdam, Maastricht University and MEDOX.nI BV.

Copyright @ Irene Slootweg, Leiden.

Pictures: Hannah Jacques.

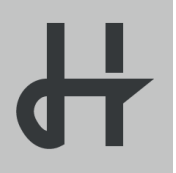

Production: Nicole Nijhuis, Gildeprint Enschede. 


\title{
Teamwork of Clinical Teachers in Postgraduate Medical Training
}

\author{
PROEFSCHRIFT \\ ter verkrijging van de graad van doctor aan de Universiteit Maastricht, \\ op gezag van de Rector Magnificus, Prof. Dr. L.L.G. Soete, \\ volgens het besluit van het College van Decanen, \\ op vrijdag 19 juni 2015, om 10.00 uur \\ door
}

Irene Arida Slootweg 


\section{Supervisors}

Prof. dr. A.J.J. Scherpbier

Prof. dr. M.J.M.H. Lombarts, Universiteit Amsterdam

Prof. dr. C.P.M. van der Vleuten

\section{Assessment Committee}

Prof. dr. L. Stassen (Chairman)

Dr. N. van Dijk, Universiteit Amsterdam

Prof. dr. J. de Graaf, Radbout Universiteit Nijmegen

Prof. dr. W.H. Gijselaers

Prof. dr. I.C. Heyligers 
Voor Irin, Sarah en Hannah

'Tel maar de sterren' 



\section{Table of Contents}

Chapter 1 General Introduction

Chapter 2 Talking about teamwork

Clinical teachers' views on how teaching teams deliver and manage residency training.

Medical Teacher, 2013;35(1):46-52.

Chapter 3 Measuring teamwork

Development and Validation of an Instrument for Measuring the Quality of Teamwork in Teaching Teams in Postgraduate Medical Training (TeamQ).

Plos one, 2014 13;9 (11).

Chapter 4 Exploring leadership in teamwork

Program directors in their role as leaders of teaching teams

in residency training.

Medical Teacher, 2014;36(12):1073-9.

Chapter 5 Speaking up in teamwork

Speaking Up among Clinical Teachers in Postgraduate

Medical Training.

(Submitted Advances in Health Sciences Education)

Chapter 6 General discussion

Chapter 7 Summary

Summary in Dutch

Dankwoord

Curriculum Vitae 



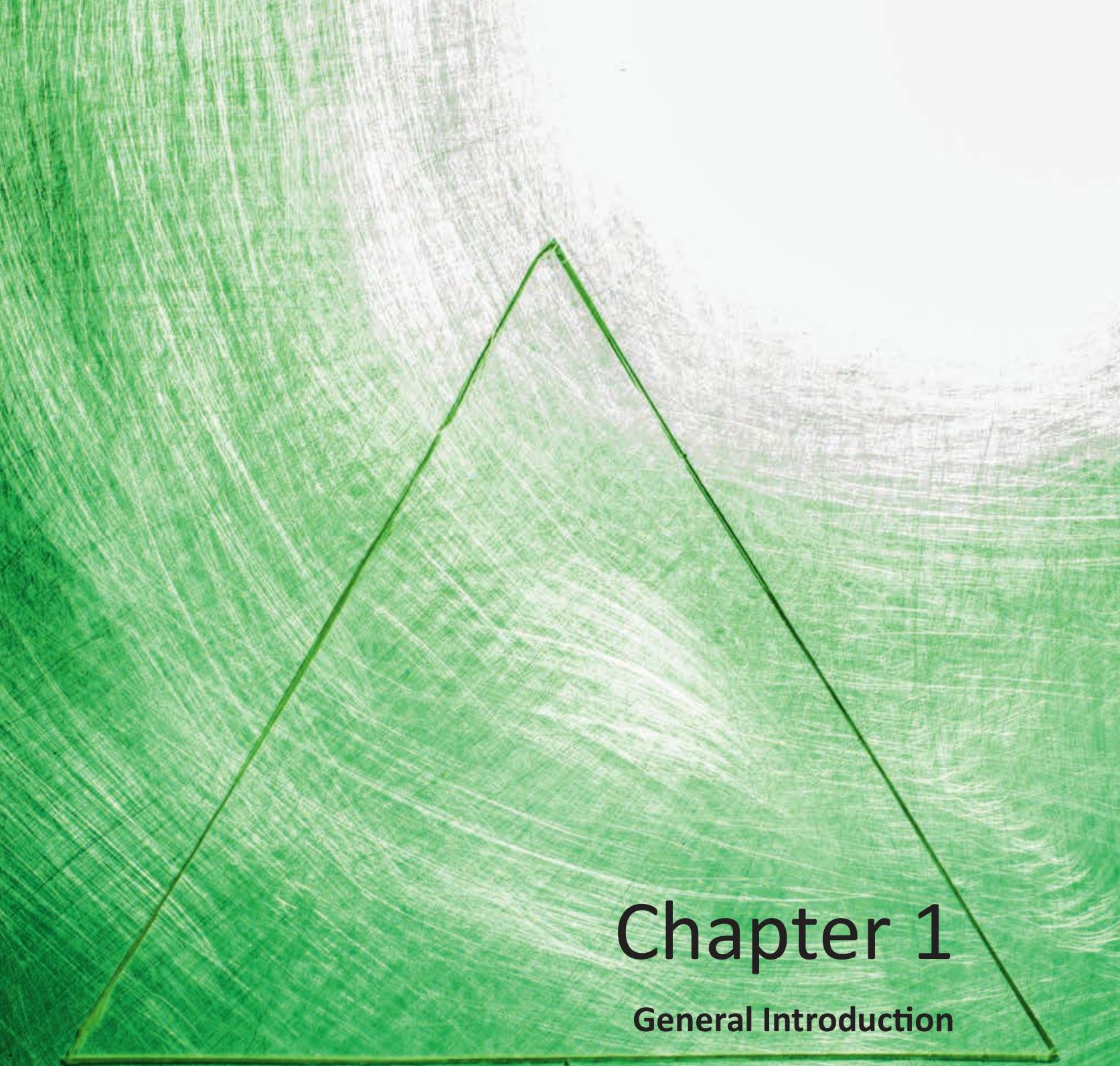


10 | Chapter 1 
Narrative during data collection:

Rehabilitation Medicine at a large university medical centre is organized in subdepartments. Together the clinical teachers are responsible for training eight residents. Teamwork is a difficult issue for this group of rehabilitation doctors, so much so that the word teamwork itself can barely be uttered in the department. A number of teambuilding activities have already taken place, but with little success. Dealing effectively with conflict, helping one another, giving and receiving feedback: all these are qualities that are almost completely absent in the department. When team members are asked why it is important for them to work together, the question is met with silence. Individual hardworking, passionate rehabilitation doctors simply do not know how to structure teamwork in postgraduate medical training. The residents are aware of the tense atmosphere: they do not hear 'we' and 'l', but 'I' and 'they'. When the residents, the clinical teachers and the program director talk about how to assure the quality of the residency training program for the coming five years, it becomes apparent that people have different ideas about teamwork. One clinical teacher wants to have more personal interaction, to get to know his colleagues better and to better work together. Another wants to be able to give more plainspoken feedback. And yet another is irritated by meetings that lead nowhere and by ineffective decision-making resulting in agreements that have been made previously not being followed up. And then there are also those clinical teachers whose main wish is simply to be left in peace.

Just as the medical profession is constantly in a state of change, postgraduate medical training is also continuously subject to new insights and transformations. Doctors today will have a competency-based training and will hopefully be better equipped to anticipate the changes within professional medical practice. ${ }^{1}$ There are two key terms for change in healthcare: patient-centred and team-based..$^{1,2}$ The recently amended and authoritative Physician Competency Framework CanMeds 2015 now incorporates the revision of the physician roles of the modern doctor in teamwork and continuing education. ${ }^{3}$ Innovations in postgraduate medical training represent an international development not only aimed at competency-based postgraduate medical training, but also with a strong focus on workplace-based assessment. ${ }^{4}$ Jointly carrying out an objective and transparent workplace-based assessment, based on the CanMeds 
competences, represents a significant challenge for clinical teachers. ${ }^{5}$ Since 2011, the joint responsibility of clinical teachers for the quality and practice of postgraduate medical training has been set out explicitly in Dutch legislation. In this legislation, clinical teachers are structured as a team and are referred to as 'members of a clinical teaching team'. The program director, a medical specialist with at least five years' clinical experience and evidently committed and motivated to teach residents, can be seen as the leader of the clinical teaching team. He or she is accountable for the quality of postgraduate medical training both to the hospital organization and the scientific medical associations. ${ }^{6}$ The question arises whether clinical teachers also experience to be members of a clinical teaching team, and how insights about teams and effective teamwork relate to the way in which clinical teachers work together in postgraduate medical training.

Residency training is organized in different ways in different parts of the world. Nonetheless, there is a single universal principle that applies throughout, namely that clinical teachers, who may also be referred to as clinician educators, are jointly responsible for the quality of the discipline-specific training of residents in the workplace. How they respond to this responsibility differs by country, by hospital and by specialty. The degree to which the clinical teachers regard teaching as an individual task or whether they consider themselves part of a teaching team also varies. It is the teamwork among clinical teachers that is explored in this thesis.

\section{Definitions}

Different definitions of teamwork are found in the literature. Researchers have used multiple terms to describe a variety of different activities and interactions among individuals, for example collaboration, collegiality, congeniality, cooperation, consultation. ${ }^{7}$ There are multiple theoretical perspectives from which teamwork can be regarded, and a number of relevant definitions are important to understand the different perspectives on which this thesis is based. ${ }^{8-12}$ The thesis addresses clinical teachers who together are responsible for training residents. To start from basics, the term 'group' is important because it applies as soon as two or more individuals are 
connected to one another by a social relationship. ${ }^{13} \mathrm{~A}$ 'group' can then be distinguished from a 'team': a 'team' is defined as a group in which two or more individuals with specified roles interact adaptively, interdependently and dynamically towards a common and valued goal. ${ }^{8,12}$ Since clinical teachers share the goal and responsibility of providing high quality postgraduate medical training, clinical teachers can be regarded as a team.

Next, it is important to understand the relevance of the process that takes place among the clinical teachers within the team. The term 'collaboration' is used to describe this. Collaboration is a process that can develop when teams work together purposively to solve a problem or make a decision, and members take ownership of and responsibility for the work of the team. ${ }^{13}$ However, just as a group is not necessarily a team, collaboration is not necessarily teamwork. ${ }^{12}$ Teamwork is more than collaboration among team members, and is defined in psychology as a set of interrelated thoughts, actions and feelings on the part of each team member that are needed to function as a team and that combine to facilitate coordinated, adaptive performance and task objectives, resulting in value-added outcomes. ${ }^{8}$ The joint responsibility for the quality and practice of postgraduate medical training based on a common goal comprises more than just resolving shared problems and making decisions together. Teamwork among clinical teachers also encompasses interrelated thoughts, actions and feelings about postgraduate medical training. This definition can be useful in determining whether teamwork and a clinical teaching team can be related here.

Finally, it is relevant to define leadership as an important construct to understand teamwork. There have been many definitions of leadership. ${ }^{8,9,14}$ Salas defines team leadership as the ability to direct and coordinate the activity of other team members, assess team performance, assign tasks, develop team knowledge, skills and abilities, motivate team members, plan and organize, and generate a positive atmosphere. ${ }^{11}$ This definition connects with the leadership role of the program director in coordinating the teaching tasks and motivating the clinical teachers for high performance in postgraduate medical training. In line with this definition, leadership by the program director focused on clinical teachers is seen as an indispensable element of teamwork in a clinical teaching team. ${ }^{15-18}$ 


\section{Theoretical perspectives}

This thesis is rooted in three theoretical perspectives. Firstly, the studies are based on the perspective of professionalism. Clinical teachers are medical professionals and the aim is to explain teamwork among clinical teachers from this viewpoint. Secondly, the studies are carried out from the perspective of social cultural learning theory, meaning that postgraduate medical training is about people learning together in the social cultural context of healthcare activities. The third theoretical perspective is that of organizational science, considering teamwork as a matter of efficiently deploying people with tasks, authorities and responsibilities. The key themes of these three perspectives will be considered in the following paragraphs.

\section{Professional perspective}

Teamwork from the perspective of the professional takes place within the continuum of the individual (characteristic, behaviors and identities), interpersonal (relations, group dynamics) and socio-institutional (economic, political) domains. ${ }^{19}$ There is interaction between these domains, where the individual influences the context and uses interpersonal relations to achieve collective professional objectives. Medical professionals anticipate the developments within the domain of healthcare and are continuously in transition. ${ }^{20,21}$ This is highlighted by a systematic review of professionalism in medical education. ${ }^{20}$ One of the conclusions from the review is that there is no consensus within the medical community about the definition of professionalism, but that there is agreement about four behaviors of professionalism: altruism, accountability, respect and integrity. ${ }^{20}$ A current description of professionalism that reflects with these conclusions is as follows: professionalism is demonstrated in everyday work through a set of behaviors that can be observed. ${ }^{22}$ These individual behaviors are necessary for the autonomous professional to be able to work in the context of inter-professional relations within healthcare. It is important to take account of these four behaviors of professionalism in this thesis on teamwork among clinical teachers as medical professionals. 


\section{Social cultural Perspective}

Teamwork from the perspective of social cultural learning theory takes place within the continuum of individual and social theory. Social cultural learning theory regards the individual learner as one aspect of a more complex activity system. ${ }^{23}$ This theory comprises different components that are dynamically linked to each other within a single system of activities. The most important component for joint learning and working in medical practice is where mind, knowledge and meaning are constructed between participants in specific situations as they work towards the collaborative achievement of a goal. ${ }^{24}$ This theory can be divided into two schools of thought, both of which address the importance of participation for the learner. The first is activity theory, defined by Vygotsky, that assumes that human activities are intrinsically social and therefore all learning is social. The second school is the communities of practice theory, that focuses more on the interaction between learners (residents) and the communities of practice (clinical teachers) where the participation in activities takes place. ${ }^{12,25}$ Participation in these communities of practice is essential for learning, both individually and collectively. It combines doing, talking, feeling and belonging and it involves our whole person, including mind, emotions, motivation and social relations. ${ }^{24,26}$ For this thesis it is important to recognise the relationship between individual learning and social learning through participation in activities.

\section{Organizational Perspective}

Teamwork from the perspective of the organization focuses on the effectiveness of tasks, responsibilities and roles. In a recent study Salas et al reviewed 138 models of team effectiveness, in which team effectiveness is seen as the evaluation of the results of interdependent performance. ${ }^{11}$ The analyses highlight the importance of team leadership behaviors in achieving team results. ${ }^{27}$ From the 138 models, the researchers developed one integral, dynamic, multilevel framework with four categories: individual characteristics, team characteristics, task characteristics and work structure. Amy Edmondson, Professor of Leadership and Management at Harvard Business School, also endorses the dynamics of teamwork and in fact treats teamwork as a verb: teaming. ${ }^{28}$ She defines teaming as a dynamic activity, determined by the mind-set and practices of teamwork, not by the design and structures of effective teams. Teaming 
involves coordinating and collaborating without the benefit of a stable team structure. The purpose of teaming is to expand knowledge and expertise so that organizations and their customers can capture mutual value. Behaviors of teaming are: (1) speaking up, (2) collaboration, (3) experimentation and (4) reflection. Teaming is 'a way to get work done while figuring out how to do it better; it is executing and learning at the same time'.28 This thesis recognises the importance of the dynamics between an effective organizational design and team results, and the vital role of the team leader.

\section{Main argument and research aim}

Teamwork in training residents seems self-evident, particularly in view of the attention paid in general to teamwork in healthcare teams. As early as 1978 the WHO emphasised that healthcare is increasingly provided by teams of health professionals. ${ }^{29}$ Inter-professional teamwork is essential within healthcare to tackle poor service delivery, improve patient safety and increase satisfaction with care. ${ }^{30}$ The critical elements of successful healthcare teams are well known from a literature review covering the period from 1990 to 2008. This review shows that enhanced clinical expertise and coordination are essential elements for teamwork in healthcare. ${ }^{31}$ There is clear evidence of a transition in teamwork among healthcare teams from seeking professional credentials to achieving care competencies for effective teamwork. ${ }^{32}$ The question is which transition is taking place in the teamwork among clinical teachers for the benefit of the quality of medical postgraduate medical training.

From the perspective of professionalism, it is well known that clinical teachers have to relate to colleagues who, based on their own professional expert role, have other ideas about training residents and about the professional standards that residents are required to meet. From the perspective of social cultural learning we know that clinical teachers, who themselves have largely been trained in the context of a traditional teacher/apprentice training paradigm, have to relate to residents who want to participate and develop competences in a social context in the workplace. From the perspective of organization science we know that clinical teachers, who are used to independently training residents, increasingly have to share the tasks, responsibilities 
and outcome of postgraduate medical training. It becomes a matter of teamwork, discussing, making decisions, managing conflict and reaching agreements. In such a situation, leadership is important, to encourage teamwork and to pay attention to the division of training tasks. ${ }^{27}$

What teamwork among clinical teachers consists of, why it is important and what it can contribute to the quality of postgraduate medical training is key to this thesis. The aim is to deconstruct the teamwork of clinical teachers, on the basis of two overarching research questions: what is the nature of teamwork among clinical teachers? and what is the role of leadership in supporting the teamwork of clinical teachers?

\section{Note on research paradigms}

This thesis takes a constructivist approach to knowledge and to the reality around us. There are a number of paradigms that act as a framework for the underlying research activities. ${ }^{33-35}$ First, there is the positivistic paradigm: 'There is one truth and it can be observed', and at the other end of the spectrum there is the constructivist paradigm: 'Multiple truths are constructed by and between people.' According to the constructivists, knowledge can be seen as a construct by the knower, while positivists see knowledge as something of value that is context-free, external to the learner. The constructivist view of the world influences the way human learning and development are regarded. ${ }^{24,36}$ In this thesis most knowledge is constructed together with the participants. Here the more post-positivistic paradigm is applied, whereby the truth is sought at that instance in time, based on the knowledge that this truth can never truly be observed.

\section{Role of researcher}

In carrying out the research described in this thesis, the ideas and assumptions of the researcher influenced the choice of research questions and the research design. In this paragraph I will reflect on and disclose my own potential biases brought into this 
thesis. Without some degree of introspection, in qualitative research referred to as reflexivity, any research is blind and purposeless. ${ }^{37}$ Reflexivity in qualitative research can be seen as an explicit self-awareness meta-analysis of the role of the researcher. I will address four variants of reflexivity that are important for critical self-reflection: introspection, mutual collaboration, social critiques, and discursive deconstruction. ${ }^{37}$ Introspection on the most important sources of interest shows that my interest in medical professional practice has grown through years of experience as a leader, adviser, trainer and designer of teaching programs within professional medical practice. This experience has taught me that everyone is able to learn, that growth and development are always possible and that worthwhile learning always involves a 'degree of pain'. Appreciative inquiry, that always assumes respect and positive development, is well-suited to this premise and is an approach that can be traced back in the way the study was carried out. ${ }^{38}$ Another variant of reflexivity is mutual collaboration. My close relationship with the target group of medical professionals is visible and recognisable in this thesis. My interest in learning from and with professionals and the power of the narratives that have been told have added colour to the research. The reflection from the viewpoint of social critiques has reinforced my conviction that there is always room for new insights and that it is always possible to engage more deeply in the scientific debate. I understand the importance of actively seeking social critiques. The discursive deconstruction is the fourth variant of reflexivity related to the ambiguity of opinions in language that we have identified in this thesis. This, more than anywhere else, is where the bridge has been sought between the language of the medical professional and the language of the clinical teacher. I feel a strong connection with the medical profession, which explains why my starting point has at all times been the language of the clinical teacher.

\section{Overview of the studies}

This introduction points out the need to study the teamwork of clinical teachers. This thesis comprises four studies that unravel this phenomenon of teamwork. The first step is the study in which the clinical teachers themselves talked about their 
experience of teamwork in postgraduate medical training. The lived experiences and views of clinical teachers are studied using the phenomenological approach (chapter 2). The next step builds on these findings in order to develop a valid and reliable instrument to evaluate the teamwork of clinical teachers (chapter 3 ). The subsequent study clarifies the role of the program directors in the teaching team and examines their experiences with strategic leadership (chapter 4). The final study adds depth to the insights on teamwork by studying 'speaking up' during a formal teaching meeting (chapter 5).

This thesis is based on the four studies described above. Given that these studies have been presented and published in an article format, it is unavoidable that some information is repeated. The intention is to use this thesis to further deconstruct teamwork in order to further contribute to the development of knowledge on teamwork and leadership. It is also intended as a means of offering guidelines to clinical teachers to improve discipline-specific teamwork in the training of residents. 


\section{References}

1. Gigerenzer G, Gray JAM.(2011). Better doctors, better patients, better decisions: Envisioning health care 2020. Cambridge: Mit Press.

2. Frank JR, Snell LS, Cate OT, et al. (2010).Competency-based medical education: theory to practice. Medical teacher, 32(8), 638-645.

3. Frank J, Snell L, Sherbino J. (2014).The Draft CanMEDS 2015: Physician Competency Framework. Royal College of Physicians and Surgeons, 1-91.

4. Scheele F, Teunissen P, Van Luijk S, et al. (2008). Introducing competency-based postgraduate medical education in the Netherlands. Medical teacher, 30(3), 248-253.

5. Sherbino J, Frank JR, Snell L. (2014). Defining the key roles and competencies of the clinician-educator of the 21st century: a national mixed-methods study. Academic medicine : journal of the Association of American Medical Colleges, 89(5),783-789.

6. Scheele F, Van Luijk S, Mulder H, et al. (2014) Is the modernisation of postgraduate medical training in the Netherlands successful? Views of the NVMO Special Interest Group on Postgraduate Medical Education. Medical teacher,36(2), 116-120.

7. $\quad$ Brouwer P. (2011) Collaboration in teacher teams. PhD report.

8. Salas E, Sims DE, Burke CS. (2005) Is there a "Big Five" in teamwork? Small Gr Res, 36(5), 555-599.

9. Mathieu J, Maynard MT, Rapp T, Gilson L. (2008) Team effectiveness 1997-2007: A review of recent advancements and a glimpse into the future. J Manage, 34(3), 410476.

10. Lemieux-Charles L, McGuire WL. (2006) What do we know about health care team effectiveness? A review of the literature. Medical Care Research and Review, 63(3), 263300.

11. Salas E. (2007) Fostering Team Effectiveness in Organizations: Toward an Integrative Theoretical Framework. In: John William Shuart BS, Will Spaulding,Jeffrey Stephen Poland, ed. Modeling complex systems. Lincoln: University of Nebraska Press.

12. Gilley A, Kerno SJ. (2010) Groups, teams, and communities of practice: A comparison. Advances in Developing Human Resources, 12(1), 46-60.

13. Franz TM. (2012) Group dynamics and team interventions: understanding and improving team performance. West Sussex, John Wiley \& Sons.

14. Avolio BJ, Walumbwa FO, Weber TJ. (2009) Leadership: current theories, research, and future directions. Annual review of psychology, 60, 421-449.

15. Gabel S. (2012) Perspective: physician leaders and their bases of power: common and disparate elements. Academic medicine : journal of the Association of American Medical Colleges, 87(2), 221-225.

16. Lieff S, Banack JG, Baker L, et al. (2013) Understanding the needs of department chairs in academic medicine. Academic medicine: journal of the Association of American Medical Colleges, 88(7), 960-966.

17. Lieff SJ, Albert M. (2010) The mindsets of medical education leaders: how do they conceive of their work? Academic medicine : journal of the Association of American Medical Colleges, 85(1), 57-62.

18. Bolman LG, Deal TE. (1992) What makes a team work? Organizational Dynamics, 21(2), 34-44.

19. Hodges BD, Ginsburg S, Cruess R, et al. (2011) Assessment of professionalism: Recommendations from the Ottawa 2010 Conference. Medical teacher, 33(5), 354-363.

20. Birden H, Glass N, Wilson I, Harrison M, Usherwood T, Nass D. (2014) Defining professionalism in medical education: a systematic review. Medical teacher, 36(1), 4761. 
21. Anderson RA, McDaniel RR, Jr. (2000) Managing health care organizations: where professionalism meets complexity science. Health care management review, 25(1), 8392.

22. Levinson W, Ginsburg S, Hafferty F, Lucey CR. (2014) Understanding medical professionalism. New York, McGraw Hill Professional.

23. Bleakley A. (2006) Broadening conceptions of learning in medical education: the message from teamworking. Medical education, 40(2), 150-157.

24. Dornan T, Mann KV, Scherpbier AJ, Spencer JA. (2011) Medical education: theory and practice. Elsevier Health Sciences.

25. Egan T, Jaye C. (2009) Communities of clinical practice: the social organization of clinical learning. Health, 13(1), 107-125.

26. Wenger E. (2000) Communities of practice and social learning systems. Organization, 7(2), 225-246.

27. Burke CS, Stagl KC, Klein C, Goodwin GF, Salas E, Halpin SM. (2006) What type of leadership behaviors are functional in teams? A meta-analysis. Leadership Quart, 17(3), 288-307.

28. Edmondson AC. (2012) Teaming: How organizations learn, innovate, and compete in the knowledge economy. Hobroken, John Wiley \& Sons.

29. Xyrichis A, Lowton K. (2008) What fosters or prevents interprofessional teamworking in primary and community care? A literature review. International journal of nursing studies, 45(1), 140-153.

30. Lewin S, Reeves S. (2011) Enacting 'team'and 'teamwork': Using Goffman's theory of impression management to illuminate interprofessional practice on hospital wards. Social Science \& Medicine, 72(10), 1595-1602.

31. Bosch M, Faber MJ, Cruijsberg J, et al. (2009) Effectiveness of patient care teams and the role of clinical expertise and coordination: a literature review. Medical Care Research and Review, 66(6suppl), 5S-35S.

32. Frenk J, Chen L, Bhutta ZA, et al. (2010) Health professionals for a new century: transforming education to strengthen health systems in an interdependent world. Lancet, 376(9756), 1923-1958.

33. Bergman E, de Feijter J, Frambach J, et al. (2012) AM last page: A guide to research paradigms relevant to medical education. Academic medicine : journal of the Association of American Medical Colleges, 87(4), 545.

34. Carter SM, Little M. (2007) Justifying knowledge, justifying method, taking action: Epistemologies, methodologies, and methods in qualitative research. Qualitative Health Research, 17(10), 1316-1328.

35. Ringsted C, Hodges B, Scherpbier A. (2011) 'The research compass': an introduction to research in medical education: AMEE Guide no. 56. Medical teacher, 33(9), 695-709.

36. Bunniss S, Kelly DR. (2010) Research paradigms in medical education research. Medical education, 44(4), 358-366.

37. Finlay L. (2002) Negotiating the swamp: the opportunity and challenge of reflexivity in research practice. Qualitative research, 2(2), 209-230.

38. Hart RK, Conklin TA, Allen SJ. (2008) Individual leader development: An appreciative inquiry approach. Advances in Developing Human Resources, 10(5), 632-650. 




\begin{abstract}
Residents learn by working in a multidisciplinary context, in different locations, with many clinical teachers. Although clinical teachers are collectively responsible for residency training, little is known about the way teaching teams function. We conducted a qualitative study to explore clinical teachers' views on how teaching teams deliver residency training. Data were collected during six focus group interviews in 2010. The analysis revealed seven teamwork themes: 1) clinical teachers were more passionate about clinical expertise than about knowledge of teaching and teamwork; 2) residents needed to be informed about clinical teachers' shared expectations; 3) the role of the program director in the teaching team needed further clarification; 4) the main topics of discussion in teaching teams were resident performance and the division of teaching tasks; 5) the structural elements of the organisation of residency training were clear; 6) clinical teachers had difficulty giving and receiving feedback; 7) clinical teachers felt under pressure to be accountable for team performance to external parties. The clinical teachers did not consider teamwork to be of any great significance to residency training. Teachers' views of professionalism and their own experiences as residents may explain their non-teamwork directed attitude. Efforts to strengthen teamwork within teaching teams may impact positively on the quality of residency training.
\end{abstract}

\title{
Practice points
}

- Social learning theory emphasises the importance of interaction and participation for learning and working.

- Teamwork of teaching teams is a dynamic process and needs attention.

- Clinical teachers are passionate about patient care and residency training but sceptical about the relevance of teamwork in teaching teams.

- Program directors should focus on developing clinical teachers'skills for working in a teaching team. 


\section{Introduction}

Residents learn by participating in patient care in the clinical workplace. Residents' learning and development can be fully understood only in relation to their involvement in the multidisciplinary context during training. ${ }^{1}$ Most tasks in the clinical workplace require several people to orchestrate their actions and work and learn together as a team. ${ }^{1}$ The importance of teamwork in patient care is generally accepted and many studies have investigated the underlying mechanisms of teamwork in the delivery of health care. ${ }^{2}$ An illustration is a study by Lingard revealing team tensions and ineffective performance in the operating room due to communication failures. ${ }^{3}$ Although different definitions of teamwork emphasise different features, there seems to be consensus about the key characteristics of effective teamwork. People working in an effective team have 1) complementary skills, 2) distinctive roles, 3) a set of shared performance goals and 4) shared norms; furthermore, they are 5) interdependent, 6) mutually accountable, 7) committed to a common goal and 8) communicate regularly with one another. ${ }^{4,5}$ Analogous to health care teams, teaching teams can be regarded as operating within a social system that is complicated and difficult to understand. In this study we focus on medical specialists in their role as clinical teachers, in which they, individually and collectively as a team, are responsible for residency training and, by implication, for the setting where future doctors receive their training. In the literature on the development of clinical teachers' teaching and assessment qualities, pitfalls have been described, such as the lack of continuity in residents' patient experience and time with teachers, and the lack of agreement among teachers about what constitutes satisfactory resident performance. ${ }^{6}$ Holmboe and colleagues also noted the importance of clinical teachers sharing information about teaching, supervision and feedback. ${ }^{6}$ The main focus of studies on the quality of residency training, however, has been on teachers' individual performance, characteristics, competencies and skills, ${ }^{7,8}$ rather than on their collaboration in teaching teams. ${ }^{2}$ Despite the abundance of research on teamwork in patient care and other industries ${ }^{9-13}$, we are not aware of any studies investigating teamwork in residency training. Assuming that insight into what makes teaching teams effective will help clinical teachers deliver high quality residency training ${ }^{14}$, we explored clinical teachers' perceptions of teamwork in relation 
to residency training. We used an approach that was inspired by the social learning theory of community of practice ${ }^{15,16}$ which characterises apprenticeship learning as a complex social relationship. A community of practice can be briefly described as a group of people who share a passion and who learn with and from each other by regular interaction while participating in practice. ${ }^{15,16}$ Our study addressed the research question: how do clinical teachers work together as a team in delivering residency training? Given the lack of scientific knowledge about teamwork of clinical teachers within the domain of postgraduate medical education, we used an open, phenomenological and exploratory approach.

\section{Methods}

\section{Context}

Residency training in the Netherlands is organized by geographical regions. In each of the eight regions, training programs are coordinated by one University Medical Center, and delivered in that center and in regional affiliated teaching hospitals where residents work alongside with and are supervised by clinical teachers. There are residency programs in 27 registered medical specialties and the total number of residents in 2011 was 6268. Annually, approximately 1000 junior doctors enter one of the programs. Each program is coordinated by a program director, who is responsible for the quality and delivery of the program and the performance of the team of clinical teachers in the participating hospitals. Program directors are appointed by the Dutch Central College of Medical Specialties (CCMS), which has initiated and is leading the current educational renovation of postgraduate residency programs in the Netherlands. These innovations are congruent with international developments towards competency-based postgraduate training programs with strong emphasis on workplace-based assessment. ${ }^{17}$

\section{Study design and study population}

We conducted a qualitative study based on principles of phenomenological theory. One of the features of this approach is rigorous interpretation of participants' 
narratives (the phenomena). This approach fits with our aim of gaining insight into the lived experiences and views of clinical teachers with regard to teamwork in teaching. We collected data in an open and exploratory manner during focus group interviews, a method that is particularly suited to elicit participants' experiences and enhance understanding of problematic areas of professional practice. ${ }^{18}$ Based on three semistructured pilot interviews with three clinical teachers, we developed three openended questions to guide the focus group discussions. The first two questions were introductory, asking about teachers' usual teaching activities and approaches that did or did not work well. The main question was: In what way do you work together with colleagues in delivering postgraduate training in your specialty? Key people from three University Medical Centers and three affiliated teaching hospitals were selected from the networks of the members of the research group ( $K L, A S, C v d V$ ), and asked to assist in composing focus groups in each of the participating hospitals. An important criterion for participation was active involvement in residency training in the hospital in question. Medical specialists received an invitational email informing them of the aim of the research and time, data and location of the focus group interview (two hours in the participants' hospitals). The focus groups were facilitated by three experienced moderators, who stimulated participants to share their personal experiences and made sure that the three guiding questions were addressed. All focus group sessions were observed by one of the researchers (IAS), tape-recorded and transcribed verbatim. Member checking was conducted by inviting all participants to comment on the summary of the discussion in their session. At the beginning of each focus group and again during member checking, the participants were asked whether they consented to the use of the data for publication. All participants gave consent. During the member checking process permission for publication was requested again, none of the participants refused.

\section{Analysis}

A phenomenological approach was used to analyse the data. Central to this approach is 'bracketing', entailing a deliberate effort to interpret materials naïvely. For 'bracketing', we used the technique of thematic networks, which provides a methodical systematization of textual data, facilitates the disclosure of each step in 
the analytic process and allows for sensitive, insightful and rich exploration of a text's overt structures and underlying patterns. ${ }^{19}$ Data analysis started with open coding by one researcher (IAS) using Atlas-Ti software for qualitative research. In order to check the coding, a second researcher coded the transcript of one of the focus group interviews. The researchers discussed differences in coding until agreement was reached. The data were further analysed using the thematic networks technique to identify three classes of themes: basic themes (lowest order with clear premises), organizing themes (grouped together, more abstract) and global themes (principal metaphors in the text as a whole). ${ }^{19}$ The thematic networks technique was then used to bracket text and identify explicit rationalisations and their implicit signification. ${ }^{19}$

\section{Results}

Between November 2009 and May 2010, we conducted six focus group interviews with a total of fifty clinical teachers from 24 specialties in three University Medical Centers and three teaching hospitals in three different regions. The number of participants was set at eight to twelve per group, but due to very high interest from potential participants we allowed sixteen participants in one of the groups. Within two days after the session, a medical student transcribed the data. Since no new information or themes were seen to emerge after six sessions, we decided that saturation was reached, and data collection was stopped. During the discussions, the participants engaged in an extensive exchange of views regarding 'best practices' for residency training. The initial list of codes, representing 220 basic themes, resulted in 68 organizing themes, which were grouped into seven global themes. The descriptions of the seven themes provide an answer to the research question regarding the teamwork of teachers in training residents. Table 1 presents short descriptions of the seven global themes with illustrative quotes. 
Table 1. The seven global themes reflecting clinical teachers' views of teamwork.

Seven Global Themes
1. The clinical teachers were more passionate
about clinical expertise than about specific
knowledge about teaching and teamwork.
2. Residents were considered to have an
apprenticeship type of relationship with
more than one medical specialist. Residents
looked for information and understanding
about clinical teachers' professional
standards and what they expected from
residents.

\section{Narrative illustrations}

P 18 'I found it only important to motivate and inspire residents and to show the beautiful aspects of our specialty'.

P 24: 'If one teacher puts the standard here and another teacher puts it there: the resident will go shopping around'.

3. The program director was deemed responsible for team performance of the clinical teachers. Neither the program director nor the other members of the teaching team had a clear idea of the director's role with regard to the development of teamwork.

4. Discussions among clinical teachers were mostly concerned with (a) residents' performance and (b) the division of teaching tasks.

5. The clinical teachers had a clear understanding of the structural elements of residency training, including formal meetings and decision-making.

6. It was not common practice for clinical teachers to use feedback in the teaching team. The teachers admitted having difficulty giving and receiving feedback in the teaching team.

7. The clinical teachers felt under external pressure to be accountable for team performance with regard to residency training.
P 17: 'Little by little everybody did their teaching tasks, because I ordered them to do so ....... I think it is important to show to residents that there is team commitment'.
P 24: 'We have a meeting every month in which we deal with the residents expeditiously'.

P2: 'We are not in favour of big changes, simply because of our structure. We have felt comfortable with the hierarchical model for a long time. It offers clarity'.

P 12: 'There are colleagues who are very grateful for feedback but they do not use it to change their behavior'.

P 16: 'The sense of urgency with regard to performance appraisal at the end of this year...I hope that when the time comes everyone will feel the urgency of this matter'.

1. The clinical teachers were proud of and passionate about their profession, their patients and the hospitals in which they worked. They supervised different residents in an apprenticeship type of relationship model. As medical professional and member of the health care team, the teachers were aware of the importance of shared professional performance goals, often laid down in clinical guidelines and protocols. The clinical teachers were more passionate about clinical expertise than about 
knowledge of education and teamwork. A shared educational vision and goals were considered to be of relatively little importance.

2. Residents were seen as being engaged in work-based learning in a multidisciplinary context, in different locations and supervised by different clinical teachers, with whom they had an apprenticeship type of relationship. The clinical teachers talked about residents' need for information about the uniform professional expectations of teachers in relation to their performance during the different stages of residency training. Residents desired to be regarded as a member of the professional team and as a future colleague. The distance between resident and clinical teacher decreased as the resident progressed through the training program. Teamwork between clinical teacher and resident could give rise to ambiguity regarding membership of the team of professionals, because one clinical teacher might see the resident as a team member, whereas another teacher might deem the resident not yet ready for that level of collegiality.

3. The program director was considered to have final responsibility for the quality of residency training and the teaching performance of the team of clinical teachers. There was a general lack of clarity among the participants about the role and position of the program director. One of the difficulties in relation to leadership and teamwork was that clinical teachers felt that improving team performance was not their responsibility but a task of the program director. They saw the program director primarily as the formal assessor of residents' performance and progress.

4. Discussions in the daily teaching practice focused mainly on the residents, such as formal assessments of residents' performance and the best teaching strategies for individual residents. Another topic was the division of teaching tasks in the team, but these discussions were often quite superficial and did not lead to clear conclusions due to clinical teachers' varying levels of commitment to teaching. For example, some of the clinical teachers regarded achievement of residents' personal learning plans as a stimulus to improve the quality of patient care, which enhance their motivation to teach residents. Others were passionate about patient care, but less interested in teaching. A shared vision on how to teach residents did not appear to be a common characteristic of teaching teams. Some participants emphasized that clinical teachers who demonstrate adherence to a shared mission with regard to teaching can serve as positive role models for residents. 
5. Elements related to the organization of the residency program, such as formal meetings and decision-making during team meetings, were clear to the clinical teachers. Residency training was mostly organized along a well-established hierarchical structure, which determined the division of teaching tasks and responsibilities. According to the clinical teachers this structure was indispensable. In large teams it could be difficult for teachers to maintain contact with one another. For the program director, team size and the physical distance between clinical teachers were crucial features in relation to effective teamwork, requiring continuous effort and creativity to organize teaching tasks and decision-making.

6. Giving and receiving peer feedback about task performance in the team was not a regular component of teamwork. Although feedback was considered essential for both teaching and teamwork, clinical teachers experienced difficulty giving and receiving peer feedback to and from other team members. Firstly, feedback was considered to be relevant to teaching: clinical teachers felt that being a good role model implied they should be able to demonstrate good feedback skills. Secondly, feedback was considered an important instrument for resolving conflicts within the team. Conflict management was seen as an important skill for any practising physician and by demonstrating these skills clinical teachers felt they could set a good example to residents.

7. Clinical teachers experienced increasing pressure at hospital, regional and national levels in relation to accountability for and transparency of team performance in the context of residency training. They were well aware of these external pressures with regard to their professional performance and the quality of residency training. The context of teamwork in residency training was considered to be increasingly important. At the hospital level, information about the quality of residency training was considered important with respect to strategic goal setting. At the regional level, dividing components of residency programs between University Medical Centers and the other teaching hospitals was considered an important issue. At the national level, the program director was considered to be accountable to the national quality inspection for the quality of the residency program. Good teamwork was considered prerequisite for shared responsibility for the quality of training programs. 


\section{Discussion}

The seven global themes in the results section together describe the phenomenon of teamwork as experienced by the clinical teachers. The results also give ample illustration of the experienced lack of effective teamwork in providing residency training. The limited role of teamwork can be fully understood when the seven themes are grouped into three categories of interrelated findings. Firstly, the clinical teachers appeared to focus mainly on individual effectiveness rather than on team effectiveness. Secondly, the clinical teachers did not use teamwork as a deliberate organisational strategy to optimise the quality of residency training. Thirdly, the lack of clarity with regard to the program director's role as team leader contributed to the lack of coherence in teams of clinical teachers. Consequently, the program director's role did not make an effective contribution to teamwork. These findings, which we will discuss in more detail later, seem to reflect clinical teachers' own experiences when training as residents in traditional, non-competency based programs. As a result of the way they were trained, today's clinical teachers are likely to see themselves primarily as medical experts who work independently most of the time, in one-on-one encounters with patients, making decisions relatively quickly and being unused to delegating work to anyone. As individual experts they are socialised to treasure their autonomy and independence and not trained to play well together in organisations. ${ }^{20}$ Many of them may have had little or no training or experience in management skills. For the present study, it is relevant to realise that the teamwork skills required for modern competency-based residency training were not acquired by today's clinical teachers when they were residents some ten to thirty years ago. Moreover, the skills the teachers learned during their training do not appear to be conducive, and sometimes even counterproductive, to effective teaching and teamwork in competency-based programs. We will now discuss the three main findings in more detail and explore ways in which effective teamwork may be promoted.

Clinical teachers as members of the teaching team

Clinical teachers may be excellent teachers individually, but this does not automatically translate into excellence in the performance of teaching teams. From the literature we know that team effectiveness differs from individual effectiveness. ${ }^{21}$ Our results show 
limitations of clinical teachers' expertise and awareness with regard to the importance of teamwork for high quality residency training. The clinical teachers appeared to be passionate about teaching medical expertise and skills to residents, but they were not very well informed about nor did they show any strong interest in knowledge about teaching and teamwork. In other words, they did not pay a great deal of attention to how they could teach together as a team. From the literature on the effectiveness of patient care teams, we know that it is essential to reinforce professionals' expertise in teamwork. ${ }^{2}$ Without this type of expertise team members cannot fulfil two crucial elements of social relations: 1) shared identity and 2) engagement with other team members. Shared identity and engagement are features of the theory of community of practice. A strong sense of shared identity involves deep connections with others through shared histories and experiences, reciprocity, affection and mutual commitments. ${ }^{16}$ Our data contained no explicit references to clinical teachers' 'shared identity', although there was evidence of shared medical knowledge and, albeit to a lesser degree, shared knowledge about teaching. Clinical teachers' limited familiarity with education as a professional domain and limited teaching expertise can be a barrier to effective teamwork in teaching. The second crucial element of community of practice is engagement with others, based on shared goals. However, in contrast to shared goals, our results indicated salient differences between clinical teachers' views of teaching. Some teachers looked upon teaching as something that was important all day and every day and regarded teamwork with colleagues as an essential component of teaching, but others saw teaching as an individual task characterised by exclusive, individual responsibility.

Feedback was an aspect of teamwork that clinical teachers considered important but difficult to perform, both within the team and in relation to residents. Feedback has been described in the literature as one of the teamwork skills that help ensure that the team achieves its purpose..$^{5}$ The confusion and lack of clarity with regard to teamwork that was found among the clinical teachers may be explained by resistance of professionals to peer and administrative review as well as by inadequate communication skills, including poor feedback skills. The theory of professionalism explains how professionalism develops and proposes encouragement of a truly professional spirit of openness. ${ }^{22}$ The climate in teaching hospitals and practice and review in practical institutes must be changed to make such openness a norm. ${ }^{23}$ We 
would therefore suggest that clinical teachers should 1) develop teamwork skills; 2) develop awareness of and expertise in teamwork; and 3) increase openness and shared engagement with residency training. In practice this means a combination of interventions, for example:

- organizing regular and structured meetings to discuss sharing teaching responsibility and reaching agreement about vision and goals of residency training;

- $\quad$ holding annual interviews between the program director and the clinical teachers about engagement in teaching and the urgency of developing teamwork skills;

- $\quad$ using valid performance evaluation instruments to increase clinical teachers' selfawareness and insight about the importance of improving teaching effectiveness and teamwork.

\section{Teamwork as a strategy for improving the quality of residency training}

Teamwork was regarded by the clinical teachers as something static, which was not amenable to change, as evidenced by the almost exclusive orientation of the clinical teachers to clinical practice and their lack of affinity with the 'teaching community'. Lemieux's theory on 'how to foster teams' stresses the importance of a focus on community and not exclusively on practice. ${ }^{9}$ Research in the past decade has shown a much greater appreciation of the temporal dynamics of teamwork, with recent studies emphasizing the critical role of developmental teamwork models, such as the concept of community of practice. Based on social learning theory, community of practice focuses on the behavior of professionals who learn and work together in practice, or, to put it differently, who participate in a two-way relationship between people and the social learning system of which they are a part. ${ }^{16}$ According to this model, people develop a particular ability through their social connections. Social learning theory describes social relations as a place where people discover reciprocity and trust. Individual behavior develops through social relations, ${ }^{24}$ which are seen as social capital because they 1) facilitate learning; 2) create meaningful connections; and 3) lead to innovation. This theory underlines the importance of clear social connections within teaching teams as a condition for the development of effective teamwork. The clinical teachers in our study, who did not appear to attach any great importance to 
the role of teamwork in residency training, made no mention at all of meaningful social connections. Moreover they did not know which colleagues to collaborate with in teaching residents or what kind of connections to build with colleagues. Also teachers' autonomous behavior seems to be an obstacle to teamwork in residency training. We therefore suggest that in developing teamwork for residency training it is important 1) to develop a shared vision and shared goals for teaching teams and 2) to clarify social connections and build trust between clinical teachers.

\section{The program director as team leader}

The program directors felt a sense of responsibility for the quality of residency training and for fostering a sense of joint responsibility within their teaching team. They tried to divide teaching tasks between team members and additionally performed many basic teaching tasks themselves. The importance of dividing tasks between team members to enhance team efficiency was described in a qualitative study on how operating room efficiency was understood in a surgical team. ${ }^{25}$ Teams became more efficient when each member had a clear understanding of his or her own responsibility and also a clear understanding of the responsibility of their colleagues. According to recent studies, leadership behaviors are person focused, such as maintaining social emotional aspects of the team, as well as task focused, such as dealing with task performance. ${ }^{12}$ With respect to the development of team skills, the program directors in our study paid more attention to teaching tasks than to the clinical teachers as team members. The seven global themes of teamwork in clinical teaching appear to be inextricably interwoven. Although they have not been trained to do so, program directors should pay attention to the dynamics of these themes. To conclude, our study reveals considerable confusion among clinical teachers about leadership of teaching teams in residency training, and we therefore suggest that program directors should 1) develop their personal leadership skills, 2) be able to navigate the seven teamwork themes, and 3) be able to steer clinical teachers towards a shared vision, goals and responsibility. This requires that program directors explore their personal leadership, set goals for improvements and frequently ask feedback on their leadership performance. Next it is important for them to plan moments of reflection and talk with a 'sparring' partner to face potential conflicts that may arise from navigation of 
the seven teamwork themes. Lastly, we advise the program director to monitor the mutual agreements and to be generous with giving feedback. ${ }^{26}$

\section{Implications for research and policy}

The results of this study may be helpful in finding ways of raising awareness among clinical teachers of the importance of teamwork for achieving high quality residency training. The insights into clinical teachers' views and experiences afforded by the results may be helpful in creating opportunities for clinical teachers to increase their knowledge about teamwork in postgraduate medical education. Improved teamwork could challenge clinical teachers to improve the quality of education, aimed at the ultimate goal of delivering high quality patient care. More research will be necessary to fully understand the dynamics of the seven global teamwork themes and to explore the most effective and efficient ways in which clinical teachers can work as a team. Based on the findings of this study we are therefore now developing an evaluation instrument for measuring teamwork in teaching teams. Finally, studies should determine whether residents learn and develop better in a learning environment with effective teamwork by their clinical teachers.

\section{Strengths and limitations}

Throughout this study the voice of the clinical teacher has been heard. The strength of our approach is that we did not adapt one of the countless models of the effectiveness of teamwork, but used an open-minded, appreciative and exploratory approach to arrive at understanding and clarification of the views and experiences of clinical. Although this study meets the criteria of the appraisal of quality research ${ }^{27}$ there are two limitations which should be noted. First, the focus group interviews were conducted in mixed groups composed of a program director and other clinical teachers, resulting in the potential disadvantage of mixed interpretations, which we were unable to disentangle. A second limitation is that the study was conducted in 
one specific setting, i.e. postgraduate specialty training in the Netherlands. We would be interested to compare our findings to those of similar research in other countries with other cultures and beliefs.

\section{Conclusions}

This study generated seven interdependent themes of teamwork for clinical teachers. Based on the results, we have made suggestions for enhancing understanding and improving the dynamics of the teamwork themes. We expect that strengthening clinical teachers' teaching and teamwork expertise can have a positive impact on teamwork in postgraduate specialty training. Moreover, program directors as leader of the teaching team, should support clinical teachers in developing mutual trust and reciprocity. In that way they may give an impulse to positively influence the medical profession's culture, in which doctors need to develop professional attitudes, knowledge and skills which equip them to work effectively together if they are to deliver safe, high-quality patient care. Clinical teachers, who take pride in their profession, may also be persuaded to fully embrace their shared responsibility for effective and efficient residency training. 


\section{References}

1. Teunissen PW. $(2010,2011)$ Learning and teaching in workplaces. In: Wilkinson TJ, 19799.Edinburgh: Churchill.

2. Bosch M, Faber MJ, Cruijsberg J, et al. (2009) Review article: Effectiveness of patient care teams and the role of clinical expertise and coordination: a literature review. Med Care Res Rev, 66(6 Suppl), 5S-35S.

3. Lingard L, Espin S, Whyte S, et al. (2004) Communication failures in the operating room: an observational classification of recurrent types and effects. Quality \& safety in health care, 13(5), 330-334.

4. Katzenbach JR, Smith DK. (1993) The discipline of teams. Harv Bus Rev, 71(2), 111-120.

5. Hammick M, Olckers L, Campion-Smith C. (2009) Learning in interprofessional teams: AMEE Guide no 38. Medical Teacher,31, 1-12.

6. Holmboe ES, Ward DS, Reznick RK, et al. (2011) Faculty development in assessment: the missing link in competency-based medical education. Academic medicine : journal of the Association of American Medical Colleges, 86(4), 460-467.

7. Boor K, Teunissen PW, Scherpbier AJJA, van der Vleuten CPM, van de Lande J, Scheele F. (2008) Residents perceptions of the ideal clinical teacher: A qualitative study. European journal of obstetrics, gynecology, and reproductive biology, 140(2), 152-157.

8. Lombarts KMJM, Bucx MJL, Arah OA. (2009) Development of a System for the Evaluation of the Teaching Qualities of Anesthesiology Faculty. Anesthesiology, 111.4, 709-716.

9. Lemieux-Charles L, McGuire WL. (2006) What do we know about health care team effectiveness? A review of the literature. Medical Care Research and Review, 63(3), 263300.

10. Xyrichis A, Lowton K. (2008) What fosters or prevents interprofessional teamworking in primary and community care? A literature review. International journal of nursing studies, 45(1), 140-153.

11. Bushe GR, Coetzer GH. (2007) Group Development and Team Effectiveness. The Journal of Applied Behavioral Science, 43(2),184-212.

12. Mathieu J, Maynard MT, Rapp T, Gilson L. (2008) Team effectiveness 1997-2007: A review of recent advancements and a glimpse into the future. J Manage, 34(3), 410476.

13. Masiello I. (2012) Why simulation-based team training has not been used effectively and what can be done about it. Advances in health sciences education : theory and practice, 17.2, 279-288.

14. Egan T, Jaye C. (2009) Communities of clinical practice: the social organization of clinical learning. Health, 13(1), 107-125.

15. Lave J, Wenger E. (1998) Communities of practice.

16. Wenger E. (2000) Communities of practice and social learning systems. Organization, 7(2), 225-246.

17. Scheele F, Teunissen P, Luijk SV, et al. (2008) Introducing competency-based postgraduate medical education in the Netherlands. Medical teache, 30(3), 248-253.

18. Barbour RS. (2005) Making sense of focus groups. Medical education, 39(7), 742-750.

19. Attride-Stirling J. (2001) Thematic networks: an analytic tool for qualitative research. Qualitative Research, 1(3), 385-405.

20. Guthrie MB. (1999) Challenges in developing physician leadership and management. Front Health Serv Manage, 15(4), 3-26.

21. Salas E. (2007) Fostering Team Effectiveness in Organizations: Toward an Integrative Theoretical Framework. In: John William Shuart BS, Will Spaulding,Jeffrey Stephen Poland, ed. Modeling complex systems. Lincoln: University of Nebraska Press. 
22. E. Freidson. (1994) Professionalism reborn. Chicago: University of Chicago Press;

23. Pronovost PJ, Freischlag JA. (2010) Improving Teamwork to Reduce Surgical Mortality. JAMA: The Journal of the American Medical Association, 304(15), 1721-1722.

24. Field J. (2005) Social connetions and long life learning. Social capital and long life learning. Bristol, The policy press.

25. Arakelian E, Gunningberg L, Larsson J. (2011) How operating room efficiency is understood in a surgical team: a qualitative study. Int I Qual Health Care, 23(1), 100106.

26. Hart RK, Conklin TA, Allen SJ. (2008) Individual leader development: An appreciative inquiry approach. Advances in Developing Human Resources, 10(5), 632-650.

27. Côté L, Turgeon J. (2005) Appraising qualitative research articles in medicine and medical education. Medical teacher, 27(1), 71-75. 




\begin{abstract}
Teamwork between clinical teachers is a challenge in postgraduate medical training. Although there are several instruments available for measuring teamwork in health care, none of them are appropriate for teaching teams. The aim of this study is to develop an instrument (TeamQ) for measuring teamwork, to investigate its psychometric properties and to explore how clinical teachers assess their teamwork. To select the items to be included in the TeamQ questionnaire, we conducted a content validation in 2011, using a Delphi procedure in which 40 experts were invited. Next, for pilot testing the preliminary tool, 1446 clinical teachers from 116 teaching teams were requested to complete the TeamQ questionnaire. For data analyses we used statistical strategies: principal component analysis, internal consistency reliability coefficient, and the number of evaluations needed to obtain reliable estimates. Lastly, the median TeamQ scores were calculated for teams to explore the levels of teamwork. In total, 31 experts participated in the Delphi study. In total, 114 teams participated in the TeamQ pilot. The median team response was 7 evaluations per team. The principal component analysis revealed 11 factors; 8 were included. The reliability coefficients of the TeamQ scales ranged from 0.75 to 0.93 .The generalizability analysis revealed that 5 to 7 evaluations were needed to obtain internal reliability coefficients of 0.70 . In terms of teamwork, the clinical teachers scored 'residents' empowerment' as the highest TeamQ scale and 'feedback culture' as the area that would most benefit from improvement. This study provides initial evidence of the validity of an instrument for measuring teamwork in teaching teams. The high response rates and the low number of evaluations needed for reliably measuring teamwork indicate that TeamQ is feasible for use by teaching teams. Future research could explore the effectiveness of feedback on teamwork in follow up measurements.
\end{abstract}




\section{Introduction}

Tackling the issue of teamwork is one of the challenges in reforming professional health education. ${ }^{1}$ This also applies to teamwork for clinical teachers in postgraduate medical training. Recent studies report that clinical teachers are more aware of the necessity for teamwork in delivering high quality residency training. ${ }^{2-4}$ In particular, they acknowledge the need to agree upon and commit to professional standards and common approaches to supervising and assessing residents, sharing educational tasks as well as assuring the quality and improvement of the training program. Teamwork is a well-researched phenomenon, where the focus is most commonly on three lines of research: the teamwork skills of individual team members, the team process and team results. ${ }^{5-7}$ In view of the collective responsibility for team results, it is important that these three research lines on teamwork are addressed. ${ }^{5,8,9}$ With regard to the first line of research, Burke presents a model for teamwork skills, including distinguishing knowledge, attitudes, traits and abilities. ${ }^{10,11}$ The second line of research, the team process, connects team members' individual teamwork skills with the team results. The team process is frequently considered to be a black box of teamwork, because it is unclear what really happens when a team member with the right teamwork skills does not achieve the right team results. ${ }^{6}$ Denecker operationalized team process indicators for multi-disciplinary teams as follows: team relations, quality of team leadership, team communication, team/task reflexivity, team vision, task orientation, team mental model, belief that multidisciplinary patient care teams result in better outcomes. ${ }^{7}$ Measuring team results, the third line of teamwork research is challenging, mainly because the results of teamwork are often unclear and can be different for individual team members. ${ }^{5,12,13}$ Outcome indicators in health care teams include teams' perceived coordination of the care process, as well as team effectiveness, teams' perceived communication with patient and family, team satisfaction, teams' perceived follow-up of the care process and professional agreement on best practices. ${ }^{14}$ The design of this study was based on the above described three lines of research on teamwork: teamwork skills, team processes and team results. More specifically, we

wanted to identify criteria for measuring teamwork skills, team processes and team results in teaching teams. Insight in actual levels of teamwork, including the strength 
and weaknesses, is a necessary first step in the process of continuous QI, also known as Quality Improvement or PDCA cycles. ${ }^{15,16}$ After this first step, evaluation, followed by reflection and improvement actions, is possible in the context of achieving or maintaining effective teamwork. Even though many measurement instruments are available for evaluating teamwork in health care teams, ${ }^{14}$ no particular instrument is specific enough for use in teaching teams in residency training. This study aims to develop and validate such an instrument and to explore how clinical teachers appraise their current levels of teamwork. More specifically, our research questions are: (i) to investigate whether teamwork in teaching teams in the context of residency training can be measured validly and reliably, and (ii) to explore how individual members of teaching teams evaluate their current levels of teamwork. To develop and validate an evaluation instrument (TeamQ), we used a mixed-methods approach based on a modified Delphi procedure, followed by psychometric analyses of the instrument.

\section{Methodology}

\section{Setting}

Postgraduate medical training in the Netherlands is organized in eight geographical regions, each of which is coordinated by one University Medical Center. In all regional affiliated hospitals, residents work alongside clinical teachers, who also act as their supervisors. Each program is coordinated by a local program director, who is responsible for the quality and delivery of the program in the workplace and the mutual performance of the clinical teachers. The clinical teacher, also named supervisor, is the medical doctor working with residents on a daily basis in the workplace, supervising and assessing the residents' medical activities, as well as teaching them professional knowledge, skills and attitudes. In most western health care systems, competencybased residency training has been introduced over the past decade. As a result, residents, in various settings, learn from a wide range of different situations under the supervision of multiple clinical teachers. This makes teamwork for supervising and assessing the residents necessary for clinical teachers. ${ }^{17}$ 
Waiver of ethical approval was provided by the Institutional Review Board of the Academic Medical Center of the University of Amsterdam, Amsterdam, The Netherlands. A waiver was provided because ethical approval for this study was not required under Dutch law.

\section{Method}

This study uses a mixed methods approach. The quantitative statistical analysis reflected in the study indicates a post-positivistic approach, a paradigm based on the assumption that there is one truth, but it can never be truly observed. A more constructivistic approach, assuming multiple truths are constructed by and between people, is reflected during the Delphi procedure and is built on stressing the frequent discussion sessions within the research group and on the dialogue with the target group of clinical teachers. ${ }^{18,19}$ We answered the first research question, that is how to validly and reliably measure teamwork, by developing the TeamQ instrument during three consecutive phases. ${ }^{20}$ The second research question, that is how clinical teachers assess their current levels of teamwork, was answered through the analysis of the available TeamQ data that also yielded the median scores per team.

\section{1) Selecting items with a Delphi procedure}

We based the definition and first selection of the teamwork items on our previous study on teamwork for clinical teachers. ${ }^{4}$ This focus group study revealed 7 preliminary teamwork themes, namely: the clinical teacher, the residents, the program director, the content, the structure, the feedback and the environment. We initially operationalized these themes into 86 teamwork items. (Table S1, Appendix). Subsequently, we performed a modified Delphi procedure. A Delphi procedure is aimed at achieving consensus among experts in a systematic manner. ${ }^{20,21}$ In a modified Delphi procedure, the items are not generated by the expert group but, as in this study, are selected based on earlier research. 4,21,22 Forty experts from diverse professional backgrounds were invited to participate by telephone or email. In total, 10 program directors, 10 educationalists, 10 supervisors and 10 residents were purposefully selected through the network of the research group. The voluntary nature of participation was emphasized in the instruction email. From August to December 2011, the 86 
items that were defined in the focus group study ${ }^{4}$ were critically reviewed during the first round of the Delphi procedure. ${ }^{20,22-24}$ In the first round the experts rated the relevance of each item on a four-point scale, from irrelevant, to highly relevant. ${ }^{21}$ We also asked the experts to give feedback on the formulation of the items and to indicate whether any particular dimensions of teamwork were underexposed. Each of the four expert groups was first analyzed separately and then combined at a later stage. The relevance of items was analyzed by calculating the mean relevance scores. These relevance scores were then plotted and inspected visually, both per expert subgroup and for all experts combined. Based on the visual inspection, items that showed consistently low relevance scores were excluded. We did not use one uniform cut-off value because of the heterogeneity between our expert subgroups. More specifically, some items were experienced as being very relevant by residents, but not by program directors and supervisors and some items about recently introduced/ renewed regulations (that will soon become very relevant in practice) were perceived as very relevant by educationalists, but not yet by supervisors or residents. Averaging all items and checking them at a uniform cut-off value would have resulted in deletion of such items. All remaining items proceeded to the second Delphi round. In this round, the experts judged items clarity on a three-point scale ( $1=$ clear, $2=$ neutral and 3 = not clear). In addition, they were asked to prioritize the items for measuring teamwork. After the second round, data were analyzed in the same way as the first round. The formulation, clarity and relevance of the items were discussed extensively in the research group. In addition, the prioritization of the various expert subgroups was included in the research group's final choice of items to be included in the TeamQ instrument. The online questionnaire was provided and answered in Dutch.

\section{2) Testing the TeamQ instrument}

To test the TeamQ instrument in practice, an internet-based environment was developed to facilitate the data collection. From January 2012 to December 2013 the instrument was offered to teaching teams from multiple specialties and multiple teaching hospitals. We use the multiple specialties and the multiple teaching hospitals to achieve an inclusive and representative sample of teaching teams. In total 116 teaching teams (1446 clinical teachers) representing 34 hospitals were invited to 
complete the TeamQ instrument. Teaching teams were approached in person, by email or through telephone contact. Teams were actively recruited using the network of the research group. Teams that were already familiar with the professional performance online program ( $w w w$.professionalperformanceonline.com), to which TeamQ was newly added, could also request use of the TeamQ in the pilot phase. Respondents were asked, in a self-reported performance assessment, to rate to what degree the situation presented in an item was valid for teamwork in their own teaching team. The measurement period lasted one month. The system was programmed to remind respondents to fill in the TeamQ questionnaire three times during this period. At the end of the measurement period, a single report summarizing the team results, was automatically generated and sent to all team members.

\section{3) Statistical analyses}

We carried out various statistical analyses to explore the validity and reliability of the TeamQ instrument. ${ }^{25-27}$ First, the number of participants that rated an item as 'I cannot judge' was calculated. Because of our heterogeneous study sample and the exploratory nature of the study, we applied a lenient cut-off of 33\%; items that were rated by over $33 \%$ as 'I cannot judge' were excluded from further analysis. Second, the data were aggregated from clinical teacher to the teaching team level. Subsequently, the median, $20^{\text {th }}$ and $80^{\text {th }}$ percentile scores of all items were calculated to inspect for extreme floor or ceiling effects. Later, a data reduction technique known as principal component analysis (PCA) was performed, to extract the number of factors (composite scales) underlying the TeamQ items. The varimax rotation method was used to extract the factors. ${ }^{28}$ We used the eigenvalue $(>1)$ criterion to determine the number of factors to extract. We also checked the scree plot. The interpretation of the factors was led by the factor loadings ( $>0.40$ ) and the meaningfulness of the factors in relation to the theory. When both were conflicting, theory was leading because of the exploratory nature of this study and the relative small sample size of our population. Third, the internal consistency reliability coefficient (Cronbach's alpha) of the composite scales extracted during the PCA was calculated. Cronbach's $\alpha$ of $>0.70$ was considered as reasonable reliability, $\alpha>0.80$ was considered as good reliability. As an additional measure of the consistency and reliability of the scales, 
the corrected item-total scale correlation was calculated for each item. Subsequently, we checked for overlap between the scales by calculating the inter-scale correlations. Ideally, inter-scale correlations are below 0.70 (which corresponds to an overlap of $<50 \%)$. Lastly, we correlated the scales with two, for this instrument developed, global items of teamwork: 'How do you rate your own contribution to the teaching team's teamwork?' and 'How do you rate this team's teamwork?' 29 These correlations provided an indication of the construct validity of the composite scales and were expected to be in the range between 0.30 and 0.80 for an indication of good construct validity. Finally, we were interested in the number of clinical teacher evaluations needed to obtain reliable scale and total scores of teamwork in teaching teams. The number of evaluations was the only random variance component of interest, so in generalizability theory terminology we had a single-facet nested design. Because generalizability theory was designed for fully crossed designs (not for nested designs), with more than two random facets, more efficient alternatives to obtain the number needed for reliable scale and total scores are available for studies with a single-faceted nested design. ${ }^{30}$ One of these alternatives is based on the assumption that the ratio of the sample size $(N)$ to the reliability coefficient $(R)$ would be approximately constant across combinations of sample size and associated reliability coefficients. ${ }^{31}$ Therefore, $R_{\text {new }}$ and $N_{\text {new }}$ can be calculated from the already known $R_{\text {old }}$ and $N_{\text {old }}$ (as observed in this study) by the formula $N_{\text {new }} / R_{\text {new }}=N_{\text {old }} / R_{\text {old }}$. In previous studies, this method yielded similar results to the computationally exhausting generalizability analysis. ${ }^{26,32}$ In this study we calculated the number of evaluations needed to obtain the pre-defined $\alpha$ coefficients of $0.60,0.70,0.80$ and 0.90 for the scales and the total score of the TeamQ. To triangulate this measure, we also calculated the observed $\alpha$ coefficients for residency training programs evaluated by 2 to 5, 6 to 9 and more than 9 team members. All analyses were performed using SPSS 20.0 for Windows.

To answer the second research question: how do individual clinical teachers evaluate their current levels of teamwork, we calculated the median score, $20^{\text {th }}$ and $80^{\text {th }}$ percentile score for all items. The clinical teachers all scored their self-reported performance of teamwork in a rating of a 5-point Likert scale ranging from 'Very low degree of application' to 'Very high degree of application'. 


\section{Results}

\section{1) Selecting items with Delphi}

The Delphi expert group consisted of 5 clinical teachers and 13 program directors. These respondents have a mean $( \pm S D)$ of $27( \pm 8)$ years clinical experience and $12( \pm 9)$ years of experience as a clinical teacher. Together with 7 residents, they represented the various surgical (12 respondents), medical (13) and auxiliary (1) specialties. In addition, 6 educationalists participated in the Delphi rounds as experts (Table 1). Based on the ratings of the 32 experts participating in the first Delphi round, evaluating relevance, 26 out of the initial 86 items were excluded. In the second Delphi round, the remaining 60 items were reviewed by 25 experts for clarity and priority (Table 1). In addition, the research group discussed the results using the three theoretical teamwork lines (individual teamwork skills, team process and team results), and decided to exclude a further 6 items. Finally, 54 items remained in the preliminary TeamQ instrument to be pilot tested in practice.

Table 1. Characteristics of the participants in the modified Delphi procedure.

\begin{tabular}{ll}
\hline Characteristics of the participants in the modified Delphi procedure. & \\
\hline Number of participants & 31 \\
Number of males & 19 \\
Number of based at an academic teaching hospital & 23 \\
Number of program directors & 13 \\
Number of clinical teachers & 5 \\
Number of residents & 7 \\
Number of educational professionals & 6 \\
\hline
\end{tabular}

\section{2) Testing TeamQ instrument}

In total, 114 teaching teams with 929 clinical teachers (64\%) used the TeamQ instrument in the pilot phase. Two teams were excluded from the analysis because only one team member responded. Team size varied from small $(<10$ team members; $42 \%$ of teams included in the study), to medium (10 - 20 team members; $46 \%$ of the teams included) to large groups (> 20 team members; $12 \%$ of teams). Of all groups, $39 \%$ were teams from surgical specialties, $46 \%$ from non-surgical and $15 \%$ from 
auxiliary disciplines. Forty percent of all teams provided postgraduate medical training in University Medical Centers (40\%) and 60\% in teaching hospitals. The median response per team was $7,20^{\text {th }}$ and $80^{\text {th }}$ percentile scores were 4 and 11 (Table 2).

Table 2. Characteristics of the participants in the testing phase of the TeamQ instrument.

\begin{tabular}{ll}
\hline Characteristics of the participants in the testing phase of the TeamQ instrument. & \\
\hline $\begin{array}{l}\text { Number of teaching teams } \\
\text { Number of clinical teachers who completed the TeamQ instrument (\% of those }\end{array}$ & $929 .(64 \%)$ \\
invited) & $7(4-11)$ \\
$\begin{array}{l}\text { Median number of evaluations completed per teaching team }\left(20^{\text {th }}-80^{\text {th }}\right. \\
\text { percentile) }\end{array}$ & 47 \\
Number of small sized teams ( $<10$ clinical teachers): & 53 \\
Number of medium sized teams (10-20 clinical teachers): & 14 \\
Number of size of large teams ( $>20$ clinical teachers): & 44 \\
Number of surgical teaching teams ${ }^{1}$ & 53 \\
Number of Non-surgical teaching teams ${ }^{2}$ & 17 \\
Number of auxiliary teaching teams & \\
Number of teaching teams based ad an University Medical Center & 46 \\
\hline
\end{tabular}

${ }^{1}$ Obstetrics/gynaecology, Surgery, Ear, nose and Throat surgery, Neurosurgery, Ophthalmology, Orthopaedic surgery, Plastic and Reconstructive surgery, Thoracal surgery.

${ }^{2}$ Dermatology, Internal Medicine, Pulmonology, Gastro-enterology, Neurology, Psychiatry, Rehabilitation Medicine, Cardiology, Paediatrics, Emergency Medicine.

${ }^{3}$ Pharmacy, Anaesthesiology, Microbiology, Nuclear medicine, Pathology, Radiology, Radiotherapy, Clinical Genetics.

\section{3) Statistical analyses}

Five items were rated as 'I cannot judge' by over a third (38\% to $53 \%$ ) of the clinical teachers. These items are listed in Table S1 and were removed before conducting the principal component analysis. Subsequently, principal component analysis (PCA) was performed on 49 items. The extraction of the items onto the composite TeamQ scales was based on factor loadings and the content of the items in relation to the theory of teamwork. Factor loadings of $>0.40$ on a composite scale were considered. When items had factor loadings of $>0.40$ on multiple scales, the items were placed in the scale where they fit best, based on 1) three theoretical research lines, ${ }^{5-7}$ or 2) highest factor loading. We reflected within the research group on these three theoretical lines by deciding which scale the 10 items with a cross loading should be placed in. 
Consequently, the PCA revealed a 10-factor structure of the TeamQ questionnaire that explained $70 \%$ of the variance among teaching teams. However, based on discussion within the research group, it was decided to exclude two factors because they contained only 2 items. One item had low factor loadings on all remaining 8 factors and based on theory this item was not essential to retain in the TeamQ instrument; therefore, this item was excluded at this stage. The remaining 8 factors (that contained a total of 48 items) were labeled as 'task expertise'; 'team expertise'; 'decision-making'; 'team leadership'; 'feedback culture'; 'team results'; 'engaging residents' and 'residents' empowerment'. The eight scales of the TeamQ contained 3 to 11 items per scale. Factor loadings and corrected item-total scale correlations are presented in Table 3 and 4. The reliability of the TeamQ scales was $\geq 0.70$ for seven scales, ranging from 0.75 for 'decision-making' to 0.93 for 'team leadership'. The scale for 'residents' empowerment' had a reliability coefficient of 0.66 .

The inter-scale correlations revealed satisfactory overlap between the scales (all $\leq$ 0.71, Table 4). The correlations between the scales and 'global item 1': 'How do you rate your own contribution to the teaching teams' teamwork?' were within the expected range $(0.30-0.80)$ for seven scales; however, the correlation was lower for the 'team leadership' scale (0.23). The correlations between 'global item 2': 'How do you rate this team's teamwork?' and the scales were all within the expected range specified above (Table 5). 


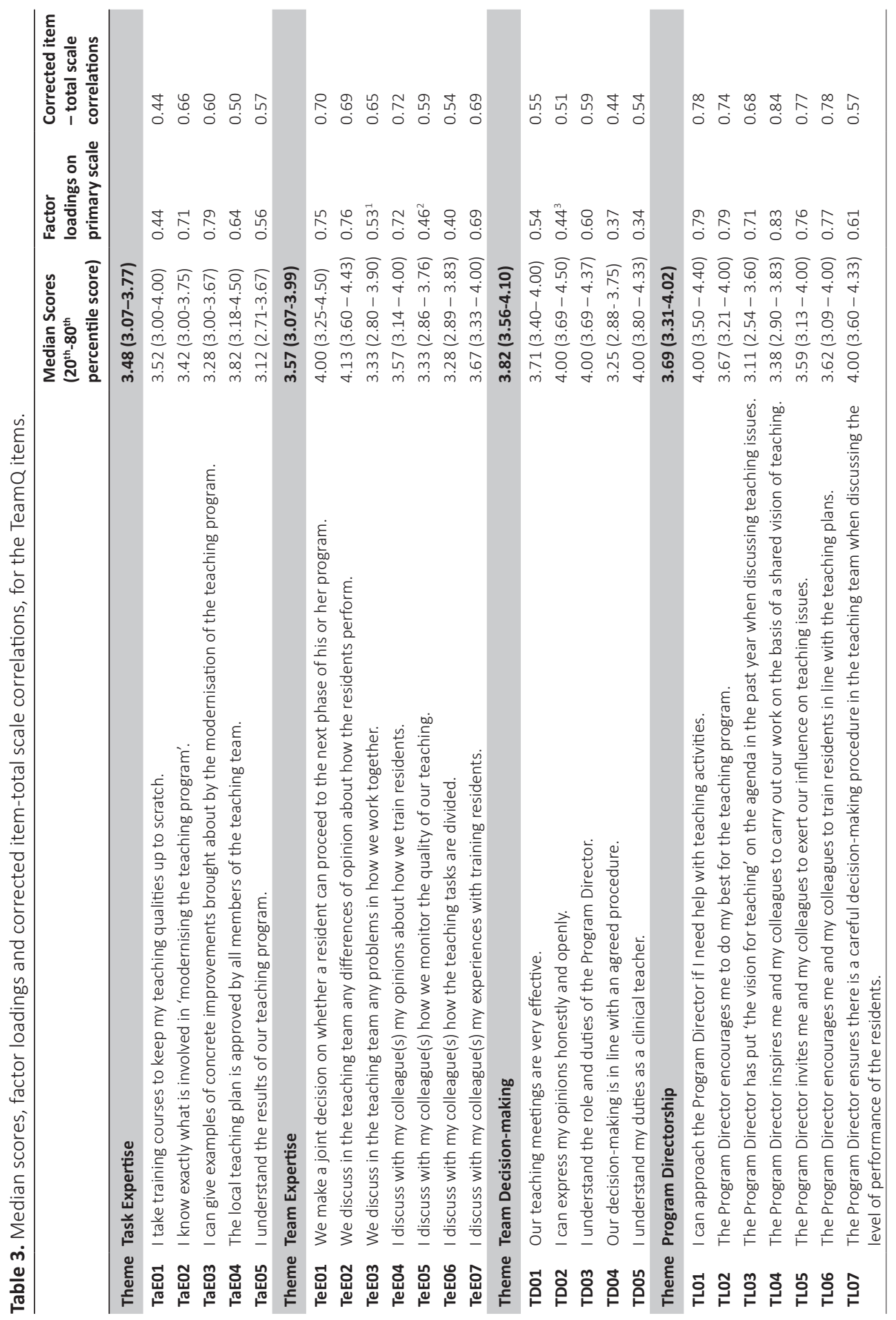




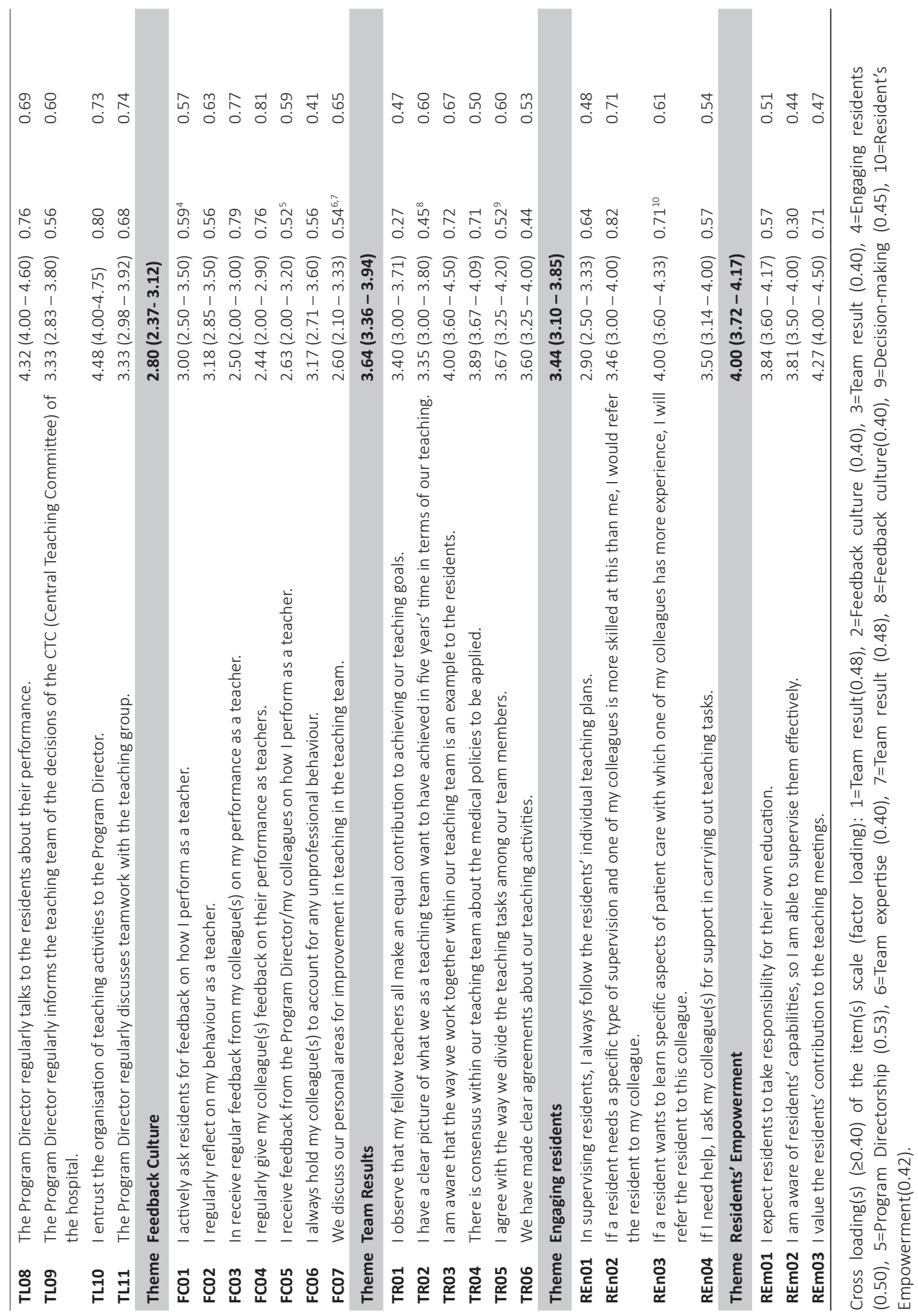


Table 4. Internal consistency reliability coefficients (Cronbach's $\alpha$ ) for all themes of the TeamQ instrument.

\begin{tabular}{ll}
\hline Theme & Cronbach's $\boldsymbol{\alpha}$ \\
\hline Task Expertise & 0.77 \\
Team Expertise & 0.87 \\
Team Decision-making & 0.75 \\
Program Directorship & 0.93 \\
Feedback Culture & 0.84 \\
Team Results & 0.80 \\
Engaging residents & 0.77 \\
Residents' Empowerment & 0.66 \\
All TeamQ items combined & 0.96 \\
\hline
\end{tabular}

Table 5. Inter-scale and scale- global item correlations of the TeamQ themes.

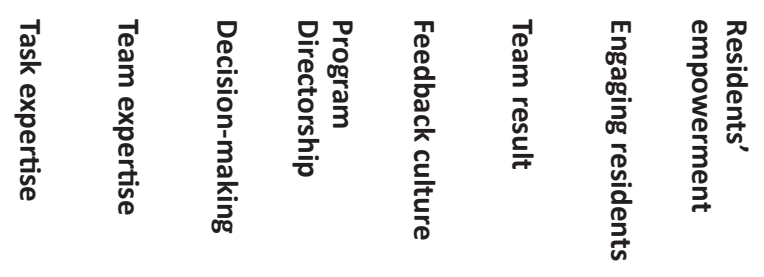

\begin{tabular}{llllllllll}
\hline Team expertise & 0.38 & 1 & - & - & - & - & - & - \\
Decision-making & 0.48 & 0.69 & 1 & - & - & - & - & - \\
Program Directorship & 0.43 & 0.40 & 0.56 & 1 & - & - & - & - \\
Feedback culture & 0.50 & 0.64 & 0.57 & 0.44 & 1 & - & - & - \\
Team result & 0.49 & 0.68 & 0.71 & 0.46 & 0.61 & 1 & - & - \\
Engaging Residents & 0.30 & 0.46 & 0.35 & 0.28 & 0.50 & 0.46 & 1 & - \\
Residents'empowerment & $0.22^{*}$ & 0.53 & 0.56 & 0.28 & 0.44 & 0.50 & 0.55 & 1 \\
$\begin{array}{l}\text { Global 1: How do you rate } \\
\text { your own contribution to the }\end{array}$ & 0.40 & 0.54 & 0.47 & $0.23^{*}$ & 0.55 & 0.64 & 0.30 & 0.44 \\
teaching team's teamwork? & & & & & & & & \\
$\begin{array}{l}\text { Global 2: How do you rate this } \\
\text { team's teamwork? }\end{array}$ & 0.40 & 0.60 & 0.50 & 0.31 & 0.44 & 0.72 & 0.36 & 0.36 \\
\hline
\end{tabular}

${ }^{*} p<0.05$ (all other correlations had $p<0.01$ )

The generalizability analysis based on the formula presented in the methods section revealed that 5 to 6 completed evaluations were needed to obtain reliability coefficients for the scale of $0.60,5$ to 7 evaluations were needed for a coefficient of $0.70,6$ to 8 evaluations were needed for a coefficient of 0.80 and 7 to 10 evaluations 
were needed for a coefficient of 0.90 . The smallest number of evaluations were needed to obtain reliable measures for the 'team leadership' scale and the greatest number were needed to obtain reliable measures for the 'residents' empowerment' scale (Table 6). The observed reliability measures of the TeamQ scales for teaching teams that completed 2 to 5 evaluations ranged from 0.69 for 'decision-making' to 0.93 for 'team leadership'. The reliability for teams that completed 6 to 9 or 10 or more evaluations was $>0.72$ for seven scales; only the 'resident empowerment' scale had low reliability levels (0.53 and 0.39 respectively) (Table 7). Figure 1 visualizes all the different steps in developing and validating TeamQ questionnaire.

Table 6. Number of completed TeamQ evaluations needed to obtain reliable theme scores, based on generalizability analysis.

\begin{tabular}{lllll}
\hline Theme & $\begin{array}{l}\text { Reliability } \\
\text { coefficient }(\boldsymbol{\alpha}) \\
\text { of } \mathbf{0 . 6 0}\end{array}$ & $\begin{array}{l}\text { Reliability } \\
\text { coefficient } \\
\text { of } \mathbf{0 . 7 0}\end{array}$ & $\begin{array}{l}\text { Reliability } \\
\text { coefficient } \\
\text { of } \mathbf{0 . 8 0}\end{array}$ & $\begin{array}{l}\text { Reliability } \\
\text { coefficient }(\boldsymbol{\alpha}) \\
\text { of } \mathbf{0 . 9 0}\end{array}$ \\
\hline Task expertise & 5 & 6 & 7 & 8 \\
\hline Team expertise & 5 & 6 & 6 & 7 \\
\hline Decision-making & 6 & 7 & 7 & 8 \\
\hline Program Directorship & 5 & 5 & 6 & 7 \\
\hline Feedback culture & 5 & 6 & 7 & 8 \\
\hline Team result & 5 & 6 & 7 & 8 \\
\hline Engaging Residents & 5 & 6 & 7 & 8 \\
\hline Residents' empowerment & 6 & 7 & 8 & 10 \\
All TeamQ items combined & 4 & 5 & 6 & 7 \\
\hline
\end{tabular}

Table 7. Observed reliability levels $(\alpha)$ for teams with a different number of completed Team $Q$ evaluations.

\begin{tabular}{llll}
\hline Theme & $\mathbf{2}$ to $\mathbf{5}$ evaluations & $\mathbf{6}$ to $\mathbf{9}$ evaluations & $\mathbf{1 0}$ or more evaluations \\
\hline Number of teams & $\mathrm{N}=44$ & $\mathrm{~N}=32$ & $\mathrm{~N}=38$ \\
Task expertise & 0.78 & 0.76 & 0.72 \\
Team expertise & 0.86 & 0.88 & 0.85 \\
Decision-making & 0.69 & 0.78 & 0.80 \\
Leadership & 0.93 & 0.93 & 0.93 \\
Feedback culture & 0.84 & 0.89 & 0.87 \\
Team result & 0.77 & 0.80 & 0.84 \\
Engaging residents & 0.72 & 0.76 & 0.80 \\
Residents'empowerment & 0.71 & 0.53 & 0.39 \\
All TeamQ items combined & 0.94 & 0.96 & 0.96 \\
\hline
\end{tabular}




\section{4) Evaluating teamwork}

Clinical teachers gave the highest median scores to the teamwork theme of 'residents' empowerment' (4.00). The scale with the lowest median score was 'feedback culture' (2.80). The other teamwork themes were all rated between 3.44 and 3.82, namely: 'task expertise' (3.48); 'team expertise' (3.57); 'decision-making' (3.82); 'team leadership' (3.69); 'team results' (3.64); 'engaging residents (3.44)(Table 3).

\section{Discussion}

This study reported how the TeamQ instrument was developed in a three-step process, resulting in a practice and theory-based, rigorously tested instrument. From the 54 initial items which were piloted in 114 teams, 48 are now included in the final TeamQ instrument and can be used for valid and reliable measurement of teamwork in teaching teams. Further, we found that clinical teachers in general positively evaluate their teamwork. The teams' feedback culture left most room for improvement. We will now discuss the answers to our two research questions by reflecting on the findings presented. We will start with discussing the results of the validation process, using the standard development and validation criteria: content validity, construct validity and internal consistency. ${ }^{33}$

First, a comprehensive and thorough analysis was conducted of the content validity of this study. Since we aimed for developing an theoretically founded instrument that was specifically fit for clinical teachers, we build on theory on teamwork and the preliminary themes and quotes from a previous focus group study of teamwork in teaching teams. ${ }^{4}$ The relevance of the preliminary items for teamwork in teaching teams was tested in a Delphi round by 31 experts. A significant number of items were excluded in this Delphi round based on limited relevance. All remaining items were rated by the experts as very relevant for evaluating teamwork in teaching teams. This contributed to the content validity of the items that were tested among 114 teaching teams in this study. The second validity criterion evaluated in this study was the construct validity. The psychometric analyses of this study revealed that the items cluster together in an 8-factor structure. The explained variance of the factors, 
the desirable correlations between the themes and the desirable correlations of the themes with the two global items of teamwork all contributed to the construct validity of the TeamQ.

We found some differences between the preliminary 7-theme structure that was based on our previous focus group study and the current 8 themes that were identified based on the psychometric analysis. This is a natural result of this exploratory phase in the validation process. The analysis presented in this study represents the first quantitative test of the preliminary structure that was based on a qualitative exploration. At that stage changes and refinement are expected and desired, while at a later stage when confirmatory techniques will be used, changes are undesirable.

The third validity criterion is evaluated the internal consistency reliability. The reliability of the TeamQ scales was found to be adequate for seven out of the eight scales, with 'team leadership' exhibiting the highest reliability and 'decision-making' the lowest. TeamQ can therefore be considered a feasible instrument for measuring teamwork in teaching teams. The 'residents' empowerment' scale had a low reliability coefficient of 0.66. The scale contains only three items, as does the 'engaging residents' scale. Having a team result that focuses clearly on the residents can be an important impetus for teamwork in teaching teams. However, as known from the literature, the result of teamwork is not always sharply defined in the minds of the team members. ${ }^{15}$ It may be necessary to employ a qualitative research method to explore in greater depth these two scales that represent the result of teamwork in teaching teams for residents.

\section{The current level of teamwork}

We also explored the research question: how individual members of teaching teams appraise their current levels of teamwork. This study shows that in general, clinical teachers evaluate their current level of teamwork positively. This study shows that clinical teachers report that their current teamwork situations are to a large extent congruent with the ideally phrased teamwork statements in the questionnaire. This suggests that they evaluate their current levels of teamwork positively. The highest and lowest scoring teamwork scales are 'residents' empowerment' and 'feedback culture'. The high score on 'residents' empowerment' may possibly be attributable to the fact that clinical teachers, although they do not see this as a result of teamwork, 
are nonetheless focused on the residents in their role as clinical teachers. The low scores on 'feedback culture' indicate the problems with feedback in teamwork of teaching teams. This is in line with another study which also reported that giving and receiving feedback is a difficult skill to master. ${ }^{34}$ Different organizational studies endorse feedback as a key element of teamwork. ${ }^{35,36}$ Through feedback, a team can obtain information about the quality and quantity of its output as well as knowledge about the effectiveness of the method used to achieve the desired levels of performance. Feedback in teamwork serves as an error detection device and thus as a stimulus to begin to identify and resolve problems. ${ }^{36}$ We suggest that, if clinical teachers develop the teamwork skills of giving and receiving feedback, the quality of assessment and supervision of the residents may improve. It may also have a positive effect on the quality of teamwork between clinical teachers in postgraduate medical training programs. ${ }^{15}$

\section{Strengths and Limitations}

We consider the combination of theory and practice and the use of both qualitative and quantitative methods in developing the TeamQ instrument as strengths of this study. The multi-center and multi-specialty character of the sample and the high response rate of the TeamQ questionnaires are also strong points. The strength of the Delphi procedure lies in the diversity of the four expert groups and the role of the research group in the modified procedure. The testing of the preliminary instrument was successful because the instrument was readily available and interested teaching teams had easy access to it. Given these strengths, we regard TeamQ as a valuable instrument for evaluating teamwork in teaching teams. However, validation must be seen as a continuous process. This study's sample did not allow for subgroup analysis, which may be considered a limitation of the study; it limits our knowledge of the applicability of TeamQ for specific situations that may benefit from more detailed analysis. Such situations could include, for example, the reliability for large and small sized groups, for different specialties and different settings. A larger sample would allow subgroup analysis in future research. 


\section{Implications for Clinical Education, Research and Policy}

Teaching teams could evaluate teamwork regularly as part of continuous improvement of the quality of post-graduate medical education 15,29 In particular, teamwork evaluations might be useful when major changes in teams occur, such as changes in team composition, or when teams are presented with major challenges, such as accreditation of residency training. Teamwork evaluations may be performed to comply with accountability requirements. In order to improve teamwork it is important to know the strengths and weaknesses of working together, but solely measuring teamwork in teaching teams does not necessarily lead to improvement. Successful implementation processes within healthcare have shown the importance of taking into account clinical teachers' readiness to change. ${ }^{15,34}$ To improve their teamwork, clinical teachers need to devote time and attention to working on the required improvements, as well as the willingness to change. Once the TeamQ evaluation has been carried out, team coaching and training can be introduced to further develop individual teamwork skills. ${ }^{10,37,38}$ Future TeamQ research should include continuous validation of the instrument to monitor and further improve the quality of the instrument and to adjust to changes in teamwork in the context of post graduate medical education. In line of this explorative validation, future research can expand evidence about convergent, predictive and concurrent validity of the TeamQ instrument. For example the TeamQ scores can be related to other quality measurement instrument and in other contexts, i.e. in different geographical, cultural and health care systems contexts.

\section{Conclusions}

This study provides a first indication of the validity and reliability of a new instrument for measuring teamwork in teaching teams in post-graduate medical training. The TeamQ instrument is now available and has been found to be reliable for use by both small and large teaching teams. The high response rates and the limited number of evaluations needed for reliably measuring teamwork indicate the feasibility of the TeamQ instrument in the evaluation of teamwork in teaching teams in practice. The 
use of TeamQ may the first step in an improvement process; indeed the TeamQ results need to be followed up by reflection and an action plan to achieve real improvement. Clinical teachers are least positive about the feedback culture in their teaching team. Facilitating the further development of individual teamwork skills, i.e. training and coaching in receiving and giving feedback, may be instrumental in realizing positive change. ${ }^{15,34}$ 


\section{References}

1. Frenk J, Chen L, Bhutta ZA, et al. (2010) Health professionals for a new century: transforming education to strengthen health systems in an interdependent world. Lancet, 376(9756), 1923-1958.

2. Jagsi R, Weinstein DF, Shapiro J, Kitch BT, Dorer D, Weissman JS. (2008) The Accreditation Council for Graduate Medical Education's limits on residents' work hours and patient safety. A study of resident experiences and perceptions before and after hours reductions. Arch Intern Med, 168(5), 493-500.

3. Frank JR, Snell LS, Cate OT, et al. (2010)Competency-based medical education: theory to practice. Medical teacher, 32(8), 638-645.

4. Slootweg I, Lombarts K, Van Der Vleuten C, Mann K, Jacobs J, Scherpbier A. (2013) Clinical teachers' views on how teaching teams deliver and manage residency training. Medical teacher, 35(1), 46-52.

5. Stewart GL. (2010) The Past Twenty Years: Teams Research Is Alive and Well at the Journal of Management. J Manage, 36(4), 801-805.

6. Mathieu J, Maynard MT, Rapp T, Gilson L. (2008) Team effectiveness 1997-2007: A review of recent advancements and a glimpse into the future. J Manage, 34(3), 410476.

7. Deneckere S, Robyns N, Vanhaecht K, et al. (2011) Indicators for Follow-Up of Multidisciplinary Teamwork in Care Processes: Results of an International Expert Panel. Eval Health Prof, 34.3, 258-277.

8. Salas E. (2007) Fostering Team Effectiveness in Organizations: Toward an Integrative Theoretical Framework. In: John William Shuart BS, Will Spaulding,Jeffrey Stephen Poland, ed. Modeling complex systems. Lincoln: University of Nebraska Press.

9. Wright MC, Phillips-Bute BG, Petrusa ER, Griffin KL, Hobbs GW, Taekman JM. (2009) Assessing teamwork in medical education and practice: relating behavioural teamwork ratings and clinical performance. Medical teacher, 31(1), 30-38.

10. Burke CS, StagI KC, Salas E, Pierce L, Kendall D. (2006) Understanding team adaptation: a conceptual analysis and model. The Journal of applied psychology, 91(6), 1189-1207.

11. DeChurch LA, Mesmer-Magnus JR. (2010) The cognitive underpinnings of effective teamwork: a meta-analysis. The Journal of applied psychology, 95(1), 32-53.

12. Neily J, Mills PD, Young-Xu Y, et al. (2010) Association between implementation of a medical team training program and surgical mortality. JAMA, 304(15), 1693-1700.

13. Senior B, Swailes S. (2007) Inside Management Teams: Developing a Teamwork Survey Instrument. British Journal of Management, 18(2), 138-153.

14. Lemieux-Charles L, McGuire WL. (2006) What do we know about health care team effectiveness? A review of the literature. Medical Care Research and Review, 63(3), 263300.

15. Edmondson AC. (2012) Teaming: How organizations learn, innovate, and compete in the knowledge economy. San Francisco,John Wiley \& Sons.

16. Varkey P, Reller MK, Resar RK. (2007) Basics of quality improvement in health care. Mayo Clinic proceedings, 82(6), 735-739.

17. Teunissen PW, Scheele F, Scherpbier AJJA, et al. (2007) How residents learn: qualitative evidence for the pivotal role of clinical activities. Medical education, 41(8), 763-770.

18. Bunniss S, Kelly DR. (2010) Research paradigms in medical education research. Medical education, 44(4), 358-366.

19. Bergman E, de FJ, Frambach J, et al. (2012) AM last page: A guide to research paradigms relevant to medical education. Academic medicine : journal of the Association of American Medical Colleges, 87(4), 545. 
20. Boor K, Van D, V, Teunissen P, Scherpbier A, Scheele F. (2011) Development and analysis of D-RECT, an instrument measuring residents? learning climate. Medical teacher, 33.10, 820-827.

21. Holey EA, Feeley JL, Dixon J, Whittaker VJ. (2007) An exploration of the use of simple statistics to measure consensus and stability in Delphi studies. BMC Med Res Methodol, $7,52$.

22. Jones J, Hunter D. (1995) Qualitative Research: Consensus methods for medical and health services research. BMJ, 311(7001), 376-380.

23. Clay-Williams R, Greenfield D, Stone J, Braithwaite J. (2014) On a wing and a prayer: an assessment of modularized crew resource management training for health care professionals. J Contin Educ Health Prof, 34(1), 56-67.

24. Albert M, Hodges B, Regehr G. (2007) Research in medical education: balancing service and science. Advances in health sciences education: theory and practice, 12(1), 103115.

25. Boerebach BC, Lombarts KM, Scherpbier AJ, Arah OA. (2013) The teacher, the physician and the person: exploring causal connections between teaching performance and role model types using directed acyclic graphs. PloS one, 8(7), e69449.

26. Lombarts KMJM, Bucx MJL, Arah OA. (2009) Development of a System for the Evaluation of the Teaching Qualities of Anesthesiology Faculty. Anesthesiology, 111.4, 709-716.

27. van der Leeuw R, Lombarts K, Heineman MJ, Arah O. (2011) Systematic evaluation of the teaching qualities of Obstetrics and Gynecology faculty: reliability and validity of the SETQ tools. PloS one, 6(5), e19142.

28. Finch H. (2006) Comparison of the Performance of Varimax and Promax Rotations: Factor Structure Recovery for Dichotomous Items. Journal of Educational Measurement, 43(1), 39-52.

29. Wenger E. (2000) Communities of practice and social learning systems. Organization, 7(2), 225-246.

30. Mushquash C, Connor B. (2006) SPSS and SAS programs for generalizability theory analyses. Behavior Research Methods, 38.3, 542-547.

31. Norman GR, Streiner DL. (2008) Biostatistics: the bare essentials. Ontario; Bc Decker Hamilton.

32. Arah OA, Hoekstra JB, Bos AP, Lombarts KM. (2011) New tools for systematic evaluation of teaching qualities of medical faculty: results of an ongoing multi-center survey. PloS one, 6(10), e25983.

33. Valentine M.A V, Nembhard IM, Edmondson A. (2011) Measuring Teamwork in Health Care Settings: a review of survey instruments. Harvard Business School, Boston, 11-116.

34. van der Leeuw M, Slootweg IA, Heineman MJ, Lombarts KMJM. (2013) Explaining how faculty members act upon residents' feedback to improve their teaching performance. Medical education, 47(11), 1089-1098.

35. DeShon RP, Kozlowski SWJ, Schmidt AM, Milner KR, Wiechmann D. (2004) A multiplegoal, multilevel model of feedback effects on the regulation of individual and team performance. Journal of Applied Psychology, 89(6), 1035-1056.

36. Nadler DA. (1979) The effects of feedback on task group behavior: A review of the experimental research. Organ Behav Hum Perf, 23(3), 309-338.

37. Edmondson A. (1999) Psychological safety and learning behavior in work teams. Administrative Science Quarterly, 44(2), 350-383.

38. Salas E, Sims DE, Burke CS. (2005) Is there a "Big Five" in teamwork? Small Gr Res, 36(5), 555-599. 


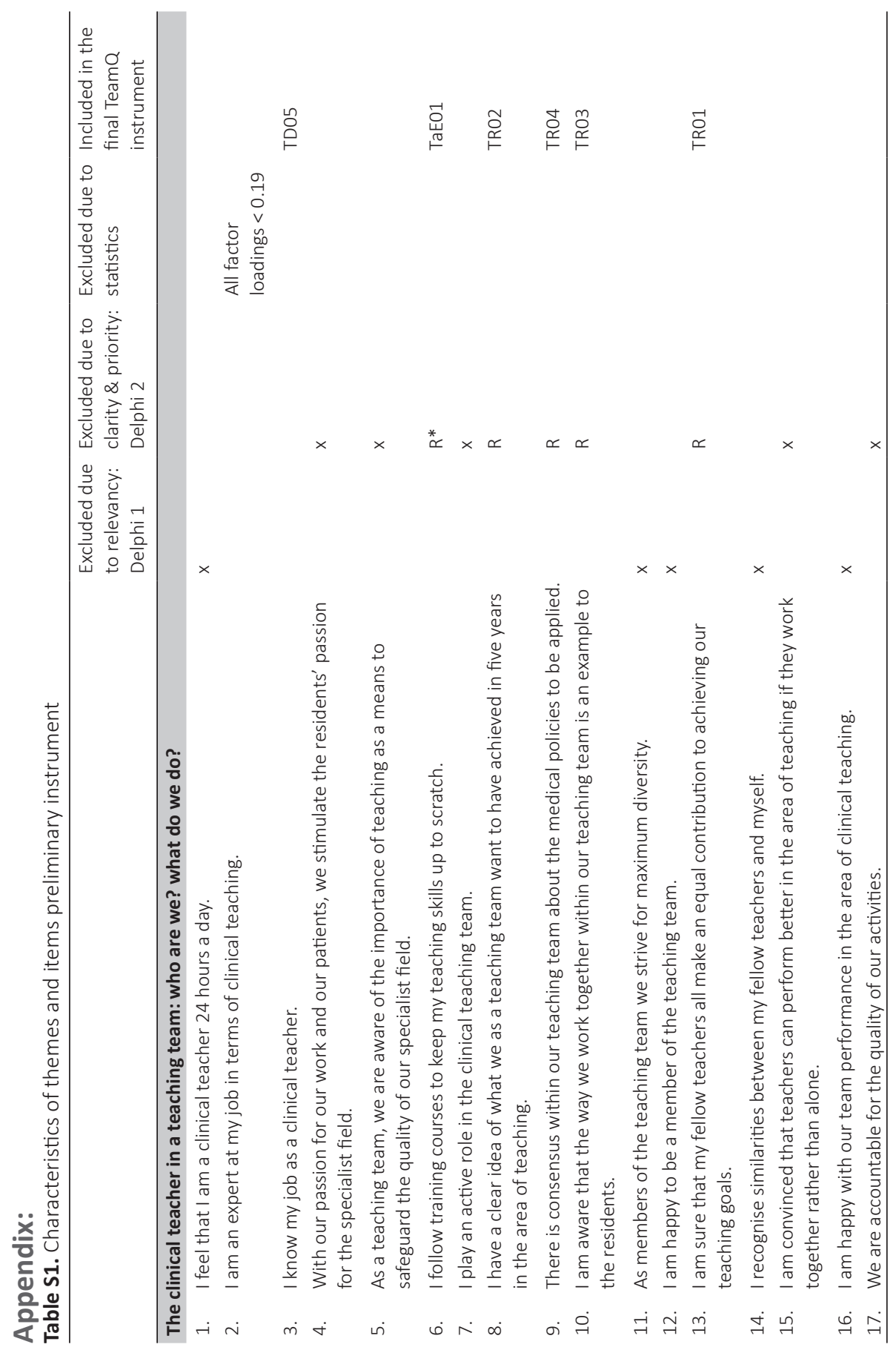




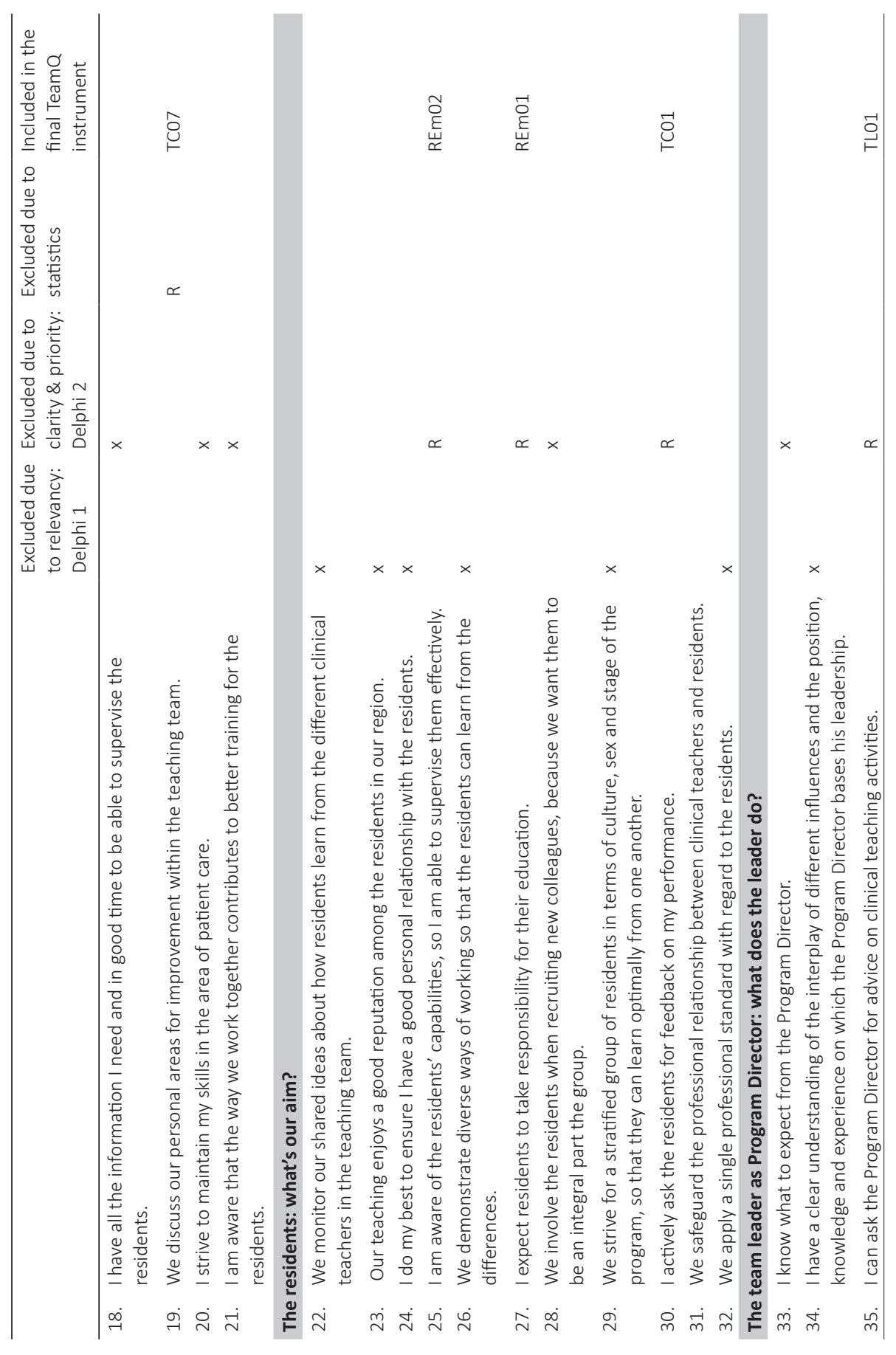




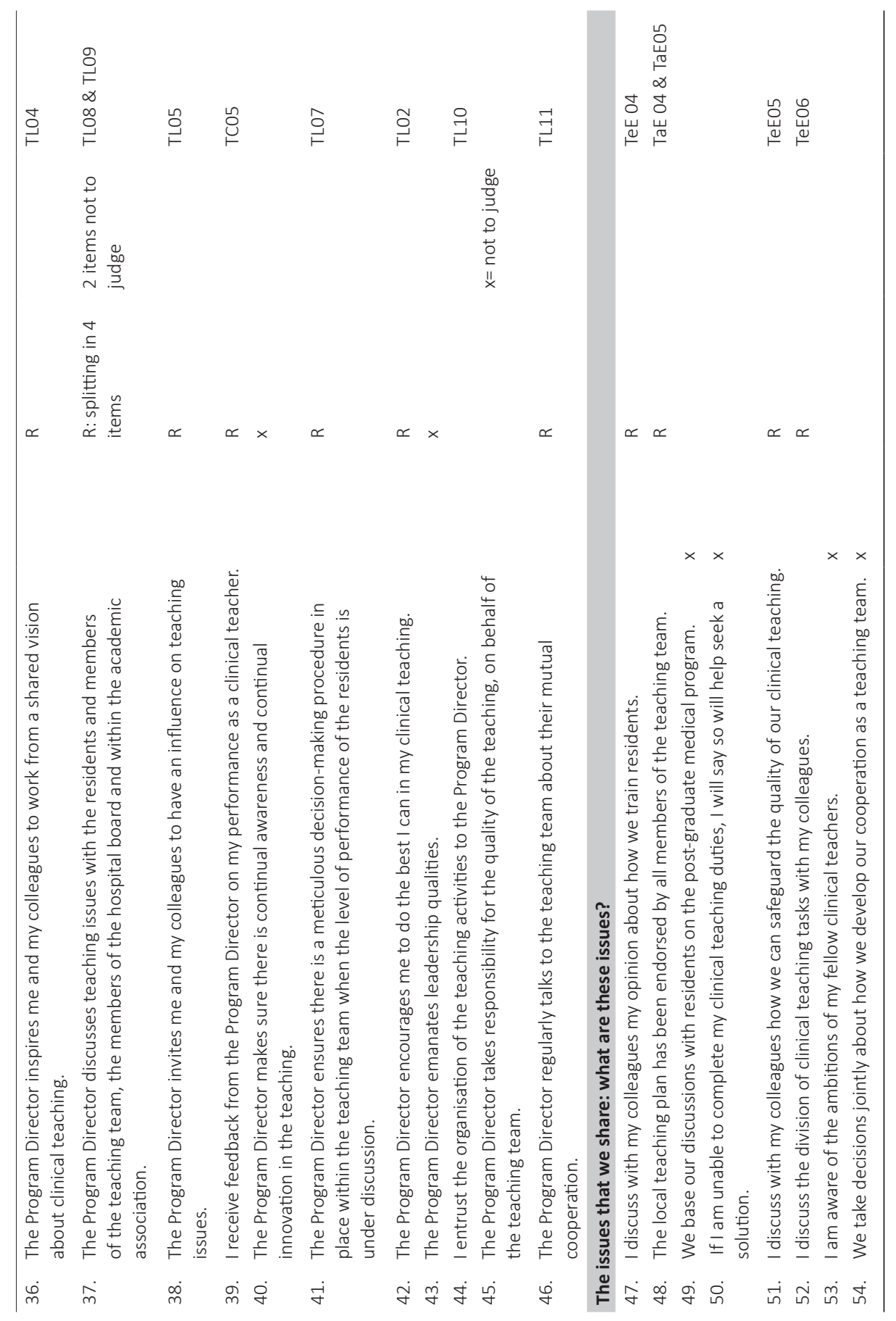




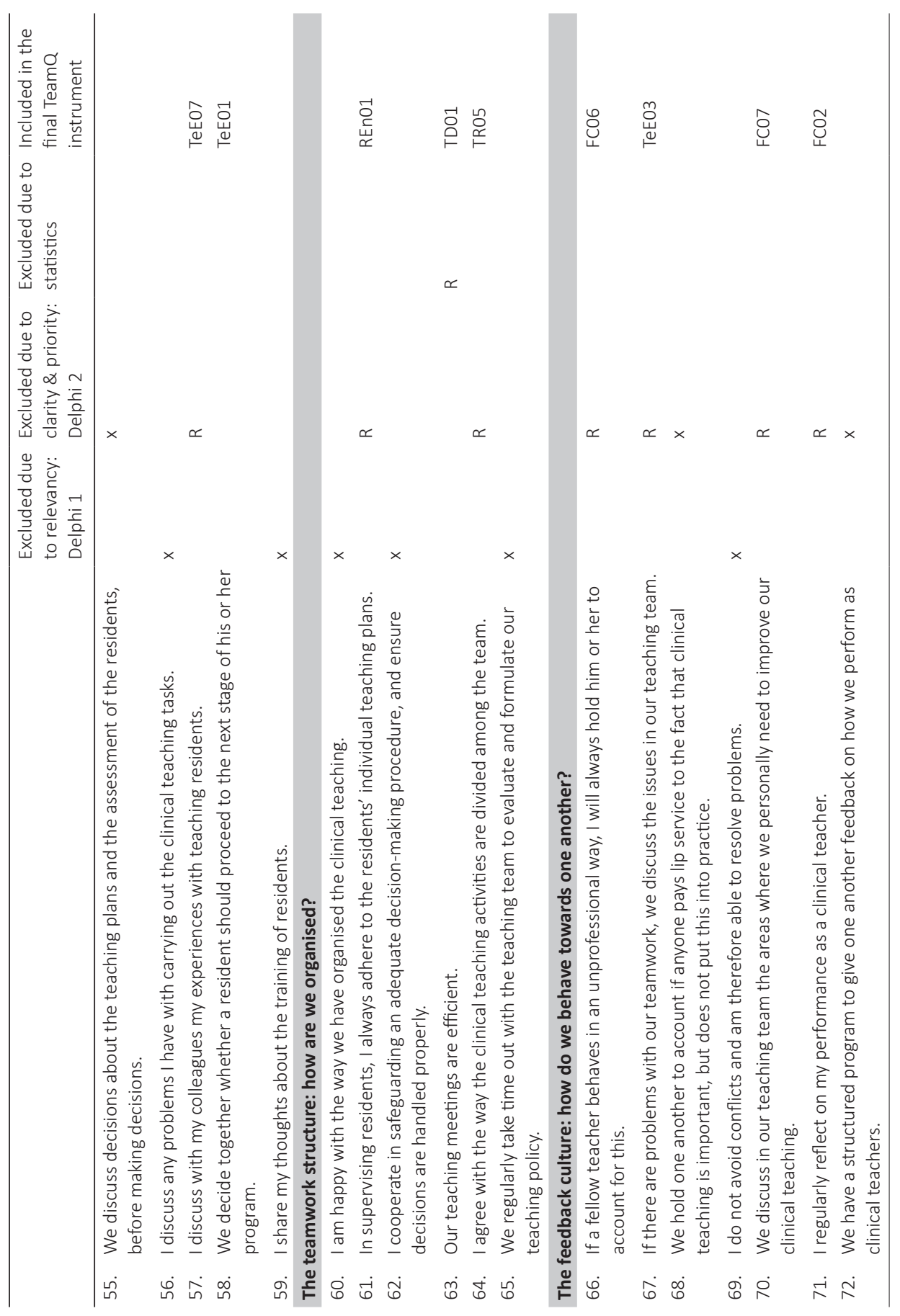




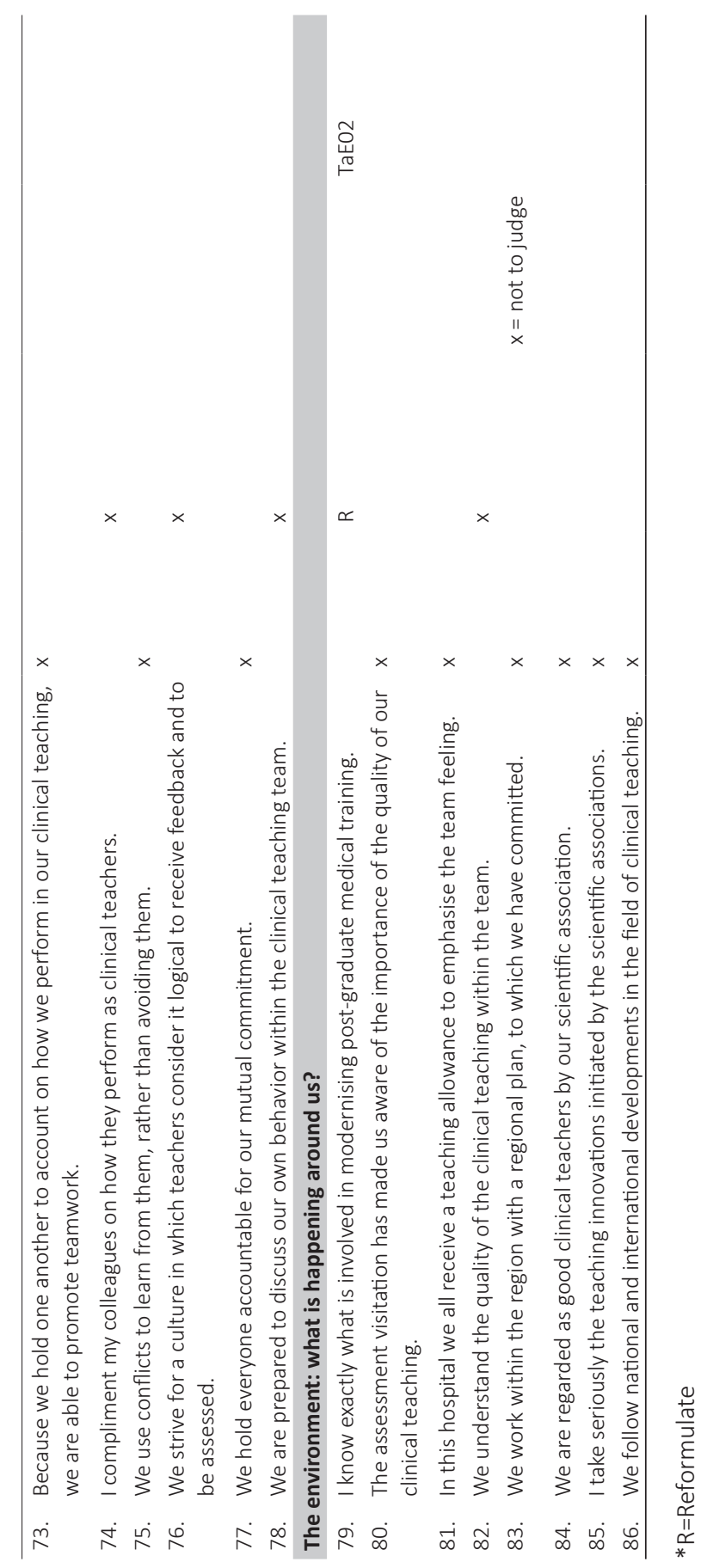






\begin{abstract}
Program directors have a formal leading position within a teaching team. It is not clear how the program directors fulfil their leadership role in practice. In this interview study we aim to explore the role of the program director as strategic leader, based on the research-question: What are the experiences of program directors with strategic leadership? We conducted an interview study using the principles of phenomenography to explore program directors' experiences. In the period June 2012 till May 2013, 16 program directors from different hospital organizations were invited for an interview. Iterative data collection and analysis were based on strategic leadership: 1) developing collective mindset, 2) focusing on collaborative learning and 3) designing teaching organization. Fourteen program directors participated in this study. We identified four leadership profiles: 1) captains, 2) carers, 3) professionals and 4) team players. The 'team players' come closest to integrally applying strategic leadership. For all four profiles there seems to be a preference for developing collectivity by means of providing information. Program directors have less experience with promoting collaborative learning and the designing of teaching organization is task-oriented. Promoting collaborative learning is the most important challenge for developing leadership within the teaching team.
\end{abstract}

\title{
Practice point
}

- Phenomenographic research helps to deeply sort out experience of strategic leadership in teaching teams.

- Strategic leadership of program directors in teaching teams can be summarized in four different profiles.

- Program directors are recommended to reflect on their leadership profile.

- Program directors could experiment with all three of the features of strategic leadership.

- Showing how to learn as a teaching team is supportive to the learning of residents. 


\section{Introduction}

Teaching residents is teamwork. ${ }^{1}$ Residents learn in various workplaces and locations from a wide range of different learning situations, under the supervision of a diverse range of teachers. ${ }^{2}$ During their training, residents need a consistent model of supervision and a clear statement about the required standard of professional practice. If these conditions are met, a safe learning environment can develop, providing residents with an optimum opportunity to learn. ${ }^{3}$ The program director, who has a formal leading position within the teaching team, has to connect the individual expectations of team members and to promote a common understanding regarding residents' expected professional performance. ${ }^{4}$ It has been suggested that program directors are more focused on the task of teaching residents than giving attention to teamwork of clinical teachers. It is not clear how program directors fulfill this leadership role in actual practice; consequently, too little is known about the activities that are needed to develop leadership skills aimed at improving collaboration within teaching teams. ${ }^{5,4}$ Although there is a wealth of information in the literature about leadership theories, leadership styles and individual leadership characteristics, ${ }^{6,7}$ little research has been done on leadership focusing specifically on the role of program directors in teaching teams. ${ }^{8-10}$ Recent research on the experiences of department chairs of schools of medicine gives us a better understanding of what leadership means and what is needed to develop leadership skills. ${ }^{7}$ McDaniel's work on strategic leadership in health care appears to best reflect the role of the program director within a teaching team. ${ }^{11}$ According to McDaniel, a strategic leader anticipates the team to external developments and exercises by: 1) developing a collective mindset, 2) focusing on collaborative learning and 3) designing the teaching organization. Promoting learning is one of the main tasks and responsibilities of teachers in order to provide a positive learning environment and to safeguard the quality of advanced specialty training. Team-based learning enables the members to develop individual team skills, work better together, and improve overall team performance. ${ }^{12,13}$ In this qualitative interview study we aim to explore deeply the role of the program director as a strategic leader. Our study is based on the following research question: What are the experiences of program directors with strategic leadership when working together 
with clinical teachers in a teaching team? The results of this exploratory study will contribute to the understanding of leadership, and can be of practical relevance in explaining and promoting the leadership role of program directors in the teamwork of teaching teams.

\section{Methodology}

This qualitative interview study is based upon the principles of a phenomenographic approach. ${ }^{14-17}$ Phenomenography is a method within qualitative research that studies the experiences of groups of people. It examines the different ways in which a given phenomenon is understood and experienced by a particular group. The focus in phenomenographic studies is on group experience and not on the individual experience. This approach is not interested in people's views, but in the way a group experiences the phenomenon under investigation. In our study, this approach allows us to understand the various ways in which the program directors experience the phenomenon of strategic leadership and how the diverse aspects of this phenomenon are inter-related. We opted for a variant on the phenomenographic approach in collecting, coding and analyzing these experiences: rather than taking an open approach to the data, we focused on the three key objectives of strategic leadership indicated previously.

The study was carried out in the Netherlands where program directors have the formal hierarchical position of head of the teaching team. Program directors have been practicing as medical specialists for a minimum of five years; they demonstrate a clear involvement in the teaching of residents, a task for which they are highly motivated.

\section{Method of data collection}

Open-ended interviews were conducted from June 2012 to May 2013. ${ }^{18,19}$ In these interviews, the participants were encouraged to speak freely about their experiences, giving concrete examples to avoid superficial descriptions about how things ought to be. ${ }^{20}$ To encourage an open attitude, we used the appreciative inquiry approach in the interviews. ${ }^{21}$ It positively invites the participant to reflect on concrete experiences of 
peak moments and shared stories of personal and organizational excellence. That is why we asked the program directors three open questions that they did not know in advance: 1) describe a successful leadership experience that you have had, 2) what metaphor best suits your leadership, and 3) describe your experiences with teamwork within the teaching team. The program directors were selected according to the method of purposeful sampling, with diversity in terms of specialty, age, gender, type of hospital organization and size of the teaching team. ${ }^{22}$

\section{Method of data coding and analysis}

All the interviews were conducted by one researcher (IAS). The interviews were recorded, transcribed, summarized and confirmed by the participants using the member check technique. The interviews were coded (IAS) in the process of data collection using Atlas-Ti, and were iteratively analyzed using the template analysis method. ${ }^{15,23}$ The initial template consisted of the three objectives formulated by McDaniel, to which the feature of 'task interpretation' was later added. ${ }^{24}$ Task interpretation reflected the variety of program director' interpretations of their leadership task. Based on discussions in the research group it was felt that the perceived differences in task interpretations were important for the analyses of the data; it was decided to add 'task interpretation' to the initial template. The template was modified following consultation within the research group and with a leadership expert. To ensure uniformity of coding and to test whether saturation had been achieved, two different researchers coded two interviews. The Ethical Review Board of the Academic Medical Center waived ethical approval for this interview study.

\section{Findings}

Fourteen of the sixteen program directors invited to take part in this interview study actually participated. Two declined to take part because of time constraints. The interviews lasted about an hour and were held at a location chosen by the program director. Table 1 gives a summary of the study population. The findings from the study of the program directors' experiences with strategic leadership are recorded in 
four descriptive profiles, within which the individual experiences are grouped. ${ }^{14}$ The program directors' metaphors used to describe their own leadership inspired us to think in terms of leadership profiles. The detailed characteristics of the profiles were then further defined using the rest of the data. We could outlined the experiences of the program directors as 'captains', 'carers', 'professionals' and 'team players' respectively (see table 2 ).

Table 1. Summary of the study population

\begin{tabular}{|c|c|}
\hline Characteristics of participating clinical teachers: & $N=14$ \\
\hline Type of specialty & $\begin{array}{l}6 \times \text { non-surgical } \\
4 \times \text { surgical } \\
4 \times \text { auxiliary }\end{array}$ \\
\hline Type of organization & $\begin{array}{l}8 \times \text { University Medical Center } \\
6 \times \text { teaching hospitals }\end{array}$ \\
\hline Sexe & $\begin{array}{l}3 \times \text { female } \\
11 \times \text { male }\end{array}$ \\
\hline Age & Range: 41 - 63; Mean 49 year; \\
\hline Years of experience as program director & Range: 3 month- 13 year; Mean 5 year \\
\hline Size of the teaching teams & Range: 6- 45; Mean 15 clinical teachers \\
\hline Number of residents & Range: 1 - 100; Mean 23 residents. \\
\hline
\end{tabular}

The program directors as captains

P4: 'I do understand why they think I'm strict: it's because I set limits on the time and the issues, on the discussions, in drawing conclusions; the way I explain it to them is that 'someone has to do it'.

This profile describes the strict, disciplined program directors who experiences leadership above all from the viewpoint of developing collectivity. This is reflected in the fact that they regularly inform the members about teaching requirements, emphasizing the rules and agreements made and reporting developments, internal and external. In their experience, information has to be reiterated repeatedly, they have to exercise patience and always need to be ahead of the game. The 'captains' design the teaching organization by organizing formal meetings and they make extensive use of email-traffic to communicate with team members. These program directors are usually well prepared before performing any task such as chairing a meeting or presenting plans. Preparation is mostly done in isolation. No explicit experiences were 
mentioned in terms of focusing on learning and creating a secure work and learning environment. These leaders hold the members to account for not keeping agreements; in doing so their primary focus is on maintaining harmony. They assume above all that the members of the teaching team are both reasonable and well mannered, and they do not like conflicts. The program directors in this profile interpret their task as unthankful. They regard the role of program director as an obligation for themselves and a duty to the medical community. With regard to developing leadership qualities, the program directors indicate that they ought to delegate more to team members. They themselves receive support in their job from the home front or from a small number of colleagues whom they trust.

\section{The program directors as carers}

P3: 'It's difficult for me to tell someone that I find it very annoying when he has

failed to do something for the third time'.

This profile describes program directors that feel a great sense of responsibility for teaching and for the teaching team. In developing collectivity they act mainly as facilitators for the team members. The program directors in this profile report to support team members to increase commitment to each other and to the teaching task by listening, show understanding and dialogue. Making timetables for teaching activities is one of the means by which they design the teaching organization. The program directors in this group want to be liked, and they constantly seek opportunities to involve the members in teaching residents. The 'carers' interpret their task as burdensome, oppressive and troublesome. Because they have such empathy for the team members, they prefer to design the teaching organization themselves rather than hold the members accountable for their responsibilities. They describe how busy the team members are with patient care and at the same time have to deal with the demands of training. Program directors within this profile regard decision-making as a form of 'begging' for loyalty for shared policy. If this loyalty is not forthcoming, the program directors become irritated and uncertain. They describe the experiences in the field of collaborative learning in terms of self-improvement. This group of program directors seeks advice of educational specialists or use peer review with colleagues. The authority that they are given is not something that they see themselves as having 
earned, but as something that they receive because the members are pleased that someone has been willing to take on the task of program director. On the subject of developing their leadership qualities, the program directors complying with this profile say that they ought to learn more about how to give constructive feedback.

\section{The program director as professional}

P 11: 'In fact, we accept that we have more or less the same vision about how to do things. This is not completely unfounded; there is certainly a shared vision, but, of course, changes in the program do mean that we sometimes have to exchange opinions with one another on particular issues'.

This profile describes a group of program directors that, as passionate medical experts, believe firmly in the importance of teaching and as such are a role model to their teaching team. In developing collectivity, they place strong emphasis on the link with the higher objective of teaching: the need to strive continuously for a high level of quality for patient care. Designing the teaching organization is not attractive for these program directors. They often discuss teaching issues in combination with consultations on complications, patient hand-over and other aspects of patient care. These program directors focus their attention on the residents; they carry out many of the teaching activities themselves and are the only group program directors to mention the importance of recruiting good residents. For them, decision-making is based on seeking a win-win situation. They talk at length about the dilemmas in managing autonomous professionals. Little or no attention is paid to learning and promoting a safe learning environment. These program directors regard the leadership as honorable. They are proud to say that the authority that they are given comes from the fact that they have earned their stripes as clinical professionals. Within this profile, the program directors seek support from a small group of like-minded colleagues. On the subject of developing their leadership qualities, they indicate that they should focus more on teamwork. 
The program directors as team players

$P$ 7: 'What's most important is that at the end of the day everyone has the feeling that it is a shared plan and a shared solution!

This profile describes the experiences of the group of program directors who regard their leadership from the viewpoint of equality. These leaders do not refer to themselves as leaders; they talk in the proudest terms about the teaching team and their task within the team. These program directors stress the importance of vigilance, monitoring and 360 degree feedback. Their main concerns in designing the teaching organization are that goals should be met and the quality of the teaching activities should not be compromised. The program directors within this profile interpret their task as a game in which opportunities and possibilities are what count most. They regard the team members as independently responsible for teaching, and have no hesitation in holding them to account for this; quite the contrary, they regard these sometimes confrontational discussions as instructive. They learn from these discussions and continue to develop as a team.

The program directors within this profile develop collectivity by focusing attention on individual members to improve their performance. They make decisions after discussing an issue at length, persuading others of the importance of the issue at hand and making use of the diversity within the team and of coalitions formed among the members. The program directors do not talk explicitly about developing leadership qualities. Nor do they describe any key experiences in their learning process, because, in their view, life itself is about learning. They are willing to listen to what experts have to say in order to improve their own performance, and team members are a source of inspiration to them. 
Table 2. Leadership profile

\begin{tabular}{|c|c|c|c|c|c|}
\hline & & Captain & Carer & Professional & Team player \\
\hline 1 & $\begin{array}{l}\text { Develops } \\
\text { collectivity by: }\end{array}$ & $\begin{array}{l}\text { Providing } \\
\text { information }\end{array}$ & $\begin{array}{l}\text { Having dialogue } \\
\text { about goals }\end{array}$ & $\begin{array}{l}\text { Emphasizing the } \\
\text { importance of high } \\
\text { quality patient care }\end{array}$ & Being vigilant \\
\hline 1.1 & $\begin{array}{l}\text { Team members } \\
\text { are: }\end{array}$ & Unaware & Unable & Autonomous & Responsible \\
\hline 1.2 & $\begin{array}{l}\text { Organizes } \\
\text { through: }\end{array}$ & Regulation & Facilitation & $\begin{array}{l}\text { Stimulating } \\
\text { participation }\end{array}$ & $\begin{array}{l}\text { Creating of } \\
\text { opportunities }\end{array}$ \\
\hline 2 & $\begin{array}{l}\text { Stimulates team } \\
\text { learning by: }\end{array}$ & $\begin{array}{l}\text { Pointing out } \\
\text { members' } \\
\text { responsibilities }\end{array}$ & $\begin{array}{l}\text { Providing } \\
\text { constructive } \\
\text { feedback }\end{array}$ & $\begin{array}{l}\text { Role modelling } \\
\text { professional } \\
\text { behaviour }\end{array}$ & $\begin{array}{l}\text { Empowering } \\
\text { team members }\end{array}$ \\
\hline 2.1 & $\begin{array}{l}\text { Develops own } \\
\text { leadership skills } \\
\text { through: }\end{array}$ & $\begin{array}{l}\text { Learning how to } \\
\text { delegate }\end{array}$ & $\begin{array}{l}\text { Learning how to } \\
\text { make decisions }\end{array}$ & $\begin{array}{l}\text { Learning how to } \\
\text { collaborate }\end{array}$ & $\begin{array}{l}\text { Learning how to } \\
\text { be a good role } \\
\text { model }\end{array}$ \\
\hline 3 & $\begin{array}{l}\text { Regards } \\
\text { anticipating the } \\
\text { environment as: }\end{array}$ & A duty & A problem & Trivial & Logical \\
\hline 4 & $\begin{array}{l}\text { Interprets tasks } \\
\text { as: }\end{array}$ & Lonely & $\begin{array}{l}\text { A big } \\
\text { responsibility }\end{array}$ & Honourable & A game \\
\hline 4.1 & Focuses on: & Me & Them & It & Us \\
\hline 4.2 & $\begin{array}{l}\text { Leadership } \\
\text { vision: }\end{array}$ & $\begin{array}{l}\text { Sticking to the } \\
\text { rules }\end{array}$ & $\begin{array}{l}\text { Increasing } \\
\text { loyalty }\end{array}$ & $\begin{array}{l}\text { The professional } \\
\text { takes the lead }\end{array}$ & $\begin{array}{l}\text { Knowledge- } \\
\text { driven }\end{array}$ \\
\hline 4.3 & State of mind: & Tough & Uncertain & Proud & Relaxed \\
\hline
\end{tabular}

\section{Discussion}

From the results of this study we learned that in order to explain the strategic leadership of program directors it is important to gain insight into how the leaders interpret their task. This finding corresponds with the study of program directors' approach to educational change, that stresses the importance of enhancing the program directors' awareness of how they interpret their task and their personal style. ${ }^{25}$ What is striking, for example, is that the 'captains' and the 'team players' have a fundamentally different vision on their team members and their leadership task's. On the one hand the 'captains' characterizing their task as 'lonely at the top'. They adopt a hierarchical approach and feel highly responsible for carrying out the necessary teaching actions, including those that stem from outside the department. 
On the other side the 'team players', who takes a more collaborative approach to leadership, are engaged in inspiring team members towards a collaborative learning process in order to improve residents' training. ${ }^{26}$ This is comparable to what Bolman e.a. found in their research, showing that different mindsets determine the way that strategic leadership is shaped. ${ }^{27}$ They report four frames through which leaders view organizations: 1) the human resource frame, that views people as an investment and their skills, attitudes, energy and commitment as a vital resource, 2) the political frame, that emphasizes building connections and managing conflict, 3) the symbolic frame, that stresses that meaning is more important than what happens, and 4) the structural frame, that stresses the importance of clear goals, roles and relationships for organizational performance. ${ }^{10}$ We could conclude that the 'captains' interpret their leadership task from the viewpoint of the structural frame. This would explain why, within the objectives of strategic leadership, their focus is much more on the design of the organization and much less on collaborative learning. The 'team players' seem to look at organizations from the human resource and political perspective. This probably explains why they handle conflicts and collaborative learning more adequately and pay much less attention to designing the organization.

If program directors were able to 'reframe their own practice' and look at the teaching practice from different mindsets, for example, by actively participating in a community of peers, ${ }^{7}$ they would gain a more complete picture of what is going on, and different ways to shape strategic leadership within the context of the three objectives would increase.

\section{Develop a collective mindset}

Within all four profiles the program directors talk about developing collectivity. The 'professionals' address this the least, while the 'carers' and 'team players' discuss this subject most explicitly. The literature is clear about the importance of developing collectivity in teams. ${ }^{5,28}$ We learned that specifically, teams with a shared purpose, social support and opportunities for participation and input from all the members are much more likely to develop shared structures. In our findings, the program directors describe their experiences with developing collectivity as: 1) providing information, without dialogue, 2) giving feedback, often on an individual bases 3) taking a decision, 
where the majority decides, and 4) feeling an enormous sense of responsibility of the leader who has difficulty delegating. It can reasonably be concluded that in terms of strategic leadership the program directors should adapt the way they develop a collective mindset. We can refer here to the literature on followership, indicating that if followers had more self-confidence and their opinions were better listened to, this would improve the opportunities for creating teamwork. To develop collectivity, it is important that leaders and followers understand one another. ${ }^{24}$ The 'carers' and 'team players' pay most attention to the diversity within the teaching team, while the 'professionals' seem to be most concerned with the autonomy of the medical specialists in their team. ${ }^{29}$

If the program directors are able to gain greater insight into the passive or active, critical or non-critical followers and can develop greater understanding of the differences between the team members who make up the followers, they will be in a better position to lead the team, which will have the effect of building collectivity.

\section{Designing the organization}

Many well-known leadership models are aimed at collaborative, shared and involved leadership, with a focus on the ability to adapt and on the way in which the team looks, or learns to look, beyond its own boundaries. ${ }^{5,30}$ From our study it appears that program directors are actively engaged in designing the organization, but this is mainly aimed at internally organizing the teaching activities. Only the 'team players', who actively work towards collaborative learning, see the opportunity to jointly anticipate the constantly changing demands from the environment. The program directors do indicate that they themselves are aware of external developments. This is, at least in part, due to the fact that within the Dutch context program directors are members of professional societies and are consequently subject to external peer review visits as part of the system of monitoring and improving the quality of teaching.

\section{Focus on learning}

In this study we opted to focus on strategic leadership because of the aspect of collaborative learning. ${ }^{11}$ It can set a strong example to residents if the members of a teaching team show that they themselves learn from mistakes and are able to devise, 
implement, evaluate and modify joint plans. Within none of the four profiles do the program directors explicitly discuss collaborative learning. It may be that this is because making mistakes and showing doubt do not fit with the image of the autonomous professional who is strongly solution focused. This leaves little room for discussion or developing shared values. We also learned how difficult it is to give one another constructive feedback. Edmondson regards promoting learning from feedback and mistakes for example, as a powerful tool for teaching and developing people. ${ }^{31-33} \mathrm{~A}$ study on the effectiveness of cardiac surgery teams shows that the most successful teams had leaders who actively managed their teams' learning efforts. The main impact on the steepness of the learning curve of a cardiac team was their engagement in realtime learning - analyzing and drawing lessons from the process while it was under way. ${ }^{34}$ Learning is difficult, because to become a learner is to become vulnerable. ${ }^{35}$ The role of the program director in creating learning is essential. This is also stressed by the theory of health care systems as Complex Adaptive Systems; a new way of thinking about connections and relationships in health care teams. To stimulate team adaptively by team-members is one of the (eight) essential key leadership skills. ${ }^{35}$ If the program directors could shift their focus onto developing collaborative learning, and become more learning-oriented leaders, the teaching team would be better able as a team to anticipate external developments and the quality of the training of residents could be expected to improve. ${ }^{36}$

\section{Strengths and limitations}

The strength of this study lies in its solid application of the methodology throughout the whole study. By promoting an open attitude and demonstrating a sincere interest in the experiences of the target group, it has been possible to gather a wealth of data. Another strength of this study is that it focused on one type of leadership, namely strategic leadership. As fewer women are program directors, there was an unequal distribution of men and women in the sample. This was a representative reflection of the target population and was therefore unavoidable. Finally, a limitation of this study is that in the interviews the program directors reflected, in words, on their 
own experiences and therefore themselves determined the meaningfulness of these experiences. We are not able to establish within the scope of this study what they actually do in practice as leaders.

\section{Conclusion}

Although all the program directors work towards the different objectives of strategic leadership, it is the 'team players' who come closest to integrally applying strategic leadership. For all four profiled leaders there seems to be a dominant preference for developing collectivity by providing information. The program directors have less experience with promoting collaborative learning. They do describe experiences in anticipating external developments, but this is primarily an internal activity and an individual action by the program directors themselves. Promoting collaborative learning is the most important challenge for developing leadership within the teaching team. This could be a means for the program directors to shift the way they interpret their task from 'lonely at the top' and burdensome, oppressive and troublesome to shared responsibility for teaching residents.

\section{Practical Implications}

This study can help the way program directors reflect on leadership on the basis of our four profiles. ${ }^{25}$ Program directors who want to develop their leadership skills could focus greater attention within the teaching team on learning with and from one another. ${ }^{36}$ Leadership skills can be learned; it is possible to learn how to resolve conflicts, to reinforce shared values and cooperative goals and to stimulate speaking up between team-members. ${ }^{37}$ There is a broad range of leadership development courses available, but the effects of these courses have not been convincingly demonstrated. ${ }^{38}$ In developing leadership skills, it is probably more effective to build communities of peers than to follow training courses, internal or external. ${ }^{38,7}$ As a team will pay more attention to develop learning, it can be expected that it will be better able to anticipate 
the continuously changing demands of the environment. Ultimately, this may have a positive effect on the teaching of residents.

\section{Future research}

This study contributes to broadening the understanding of strategic leadership by stressing the importance of how leaders experience and interpret their task. This finding is in line with the literature by Edmondson that maintains that paying attention to the environment and having concern for internal factors have a positive influence on one another. ${ }^{31}$ Our study covered the benefits of strategic leadership; we also identified the need to gain greater insight into the causal relations between leadership and outcome. ${ }^{24}$ In addition, the four profiles that describe the experiences of the program directors raise questions for further research on characteristics that fit within the profiles. Once these questions have been answered, the profiles can form the basis for learning more about leadership in teams. ${ }^{39,40}$ 


\section{References}

1. Holmboe ES, Ward DS, Reznick RK, et al. (2011) Faculty development in assessment: the missing link in competency-based medical education. Academic medicine : journal of the Association of American Medical Colleges, 86(4), 460-467.

2. Teunissen PW, Scheele F, Scherpbier AJJA, et al. (2007) How residents learn: qualitative evidence for the pivotal role of clinical activities. Medical education, 41(8), 763-770.

3. Teunissen PW. $(2010,2011)$ Learning and teaching in workplaces. Wilkinson TJ, 197-99 Edinburgh, Churchill.

4. Scheele F, Van Luijk S, Mulder H, et al. (2014) Is the modernisation of postgraduate medical training in the Netherlands successful? Views of the NVMO Special Interest Group on Postgraduate Medical Education. Medical teacher, 36(2), 116-120.

5. Leasure EL, Jones RR, Meade LB, et al. (2013) There is no " $\mathrm{i}$ " in teamwork in the patientcentered medical home: defining teamwork competencies for academic practice. Academic medicine: journal of the Association of American Medical Colleges, 88(5), 585-592.

6. Gabel S. (2012) Perspective: physician leaders and their bases of power: common and disparate elements. Academic medicine : journal of the Association of American Medical Colleges, 87(2), 221-225.

7. Lieff S, Banack JG, Baker L, et al. (2013) Understanding the needs of department chairs in academic medicine. Academic medicine: journal of the Association of American Medical Colleges, 88(7), 960-966.

8. Mathieu J, Maynard MT, Rapp T, Gilson L. (2008) Team effectiveness 1997-2007: A review of recent advancements and a glimpse into the future. J Manage, 34(3), 410476.

9. Salas E, Sims DE, Burke CS. (2005) Is there a "Big Five" in teamwork? Small Gr Res, 36(5), 555-599.

10. Lieff SJ, Albert M. (2010) The mindsets of medical education leaders: how do they conceive of their work? Academic medicine : journal of the Association of American Medical Colleges, 85(1), 57-62.

11. McDaniel RRJ. (1997) Strategic Leadership: A View from Quantum and Chaos Theories. Health Care Management Review Winter, 22(1):21-37.22(1), 21-37.

12. Magrane D, Khan O, Pigeon Y, Leadley J, Grigsby RK. (2010) Learning about teams by participating in teams. Academic medicine : journal of the Association of American Medical Colleges, 85(8), 1303-1311.

13. Hammick M, Olckers L, Campion-Smith C. (2009) Learning in interprofessional teams: AMEE Guide no 38. Medical Teacher, 31, 1-12.

14. Stenfors-Hayes T, Hult H, Dahlgren LO. (2011) What does it mean to be a good teacher and clinical supervisor in medical education? Advances in health sciences education : theory and practice, 16(2), 197-210.

15. Stenfors-Hayes T, Hult H, Dahlgren MA. (2013) A phenomenographic approach to research in medical education. Medical education, 47(3), 261-270.

16. Starks H, Brown Trinidad S. (2007) Choose Your Method: A Comparison of Phenomenology, Discourse Analysis, and Grounded Theory. Qualitative Health Research, 17(10), 1372-1380.

17. Marton F. (1981) Phenomenography-describing conceptions of the world around us. Instructional science, 10(2), 177-200.

18. Britten N. (1995) Qualitative research: qualitative interviews in medical research. BMJ, 311(6999), 251-253.

19. Cassell C. (2005) Creating the interviewer: identity work in the management research process. Qualitative research, 5(2), 167-179. 
20. Larsson J, Holmström I. (2007) Phenomenographic or phenomenological analysis: does it matter? Examples from a study on anaesthesiologists' work. International Journal of Qualitative Studies on Health and Well-being, 2(1), 55-64.

21. Watkins JM, Mohr BJ, Kelly R. (2011) Appreciative inquiry: Change at the speed of imagination. Vol 35: Hoboken, John Wiley \& Sons.

22. Kuper A, Reeves S, Levinson W. (2008) An introduction to reading and appraising qualitative research. BMJ.,337.

23. King N, Carroll C, Newton P, Dornan T. (2002) "You can't Cure it so you have to Endure it": The Experience of Adaptation to Diabetic Renal Disease. Qualitative Health Research, 12(3), 329-346.

24. Avolio BJ, Walumbwa FO, Weber TJ. (2009) Leadership: current theories, research, and future directions. Annual review of psychology, 60, 421-449.

25. Fokkema JP, Westerman M, Teunissen PW, et al. (2012) How lead consultants approach educational change in postgraduate medical education. Medical education, 46(4), 390398.

26. Lingard L, McDougall A, Levstik M, Chandok N, Spafford MM, Schryer C. (2012) Representing complexity well: a story about teamwork, with implications for how we teach collaboration. Medical education, 46(9), 869-877.

27. Bolman LG, Deal TE. (1991) Leadership and management effectiveness: a multiframe, multisector analysis. Human Resource Management, 30(4), 509-534.

28. Leslie K, Baker L, Egan-Lee E, Esdaile M, Reeves S. (2013) Advancing faculty development in medical education: a systematic review. Academic Medicine, 88(7), 1038-1045.

29. Birden H, Glass N, Wilson I, Harrison M, Usherwood T, Nass D. (2014) Defining professionalism in medical education: a systematic review. Medical teacher, 36(1), 4761.

30. Swanwick T, McKimm J. (2011) What is clinical leadership...and why is it important? Clin Teach, 8(1), 22-26.

31. Edmondson AC. (2012) Teaming: How organizations learn, innovate, and compete in the knowledge economy. San Francisco, John Wiley \& Sons.

32. Edmondson AC. (1999) Psychological safety and learning behavior in work teams. Administrative Science Quarterly, 44(2), 350-383.

33. Edmondson AC. (2003) Speaking up in the operating room: How team leaders promote learning in interdisciplinary action teams. J Manage Stud, 4O(6), 1419-1452.

34. Edmondson AC, Bohmer R, Pisano G. (2001) Speeding up team learning. Harvard business review, 79(9), 125-134.

35. Anderson RA, McDaniel RR, Jr. (2000) Managing health care organizations: where professionalism meets complexity science. Health care management review, 25(1), 8392.

36. Singer SJ, Hayes J, Cooper JB, et al. (2011) A case for safety leadership team training of hospital managers. Health care management review, 36(2), 188-200.

37. Mitchell R, Parker V, Giles M, Boyle B. (2014) The ABC of health care team dynamics: Understanding complex affective, behavioral, and cognitive dynamics in interprofessional teams. Health care management review, 39.1, 1-9.

38. Straus SE, Soobiah C, Levinson W. (2013) The impact of leadership training programs on physicians in academic medical centers: A systematic review. Academic Medicine, 88(5), 710-723.

39. Steinert Y, Naismith L, Mann K. (2012) Faculty development initiatives designed to promote leadership in medical education. A BEME systematic review: BEME Guide No. 19. Medical teacher, 34(6), 483-503.

40. Lingard L, Vanstone M, Durrant M, et al. (2012) Conflicting messages: examining the dynamics of leadership on interprofessional teams. Academic medicine : journal of the Association of American Medical Colleges, 87(12), 1762-1767. 



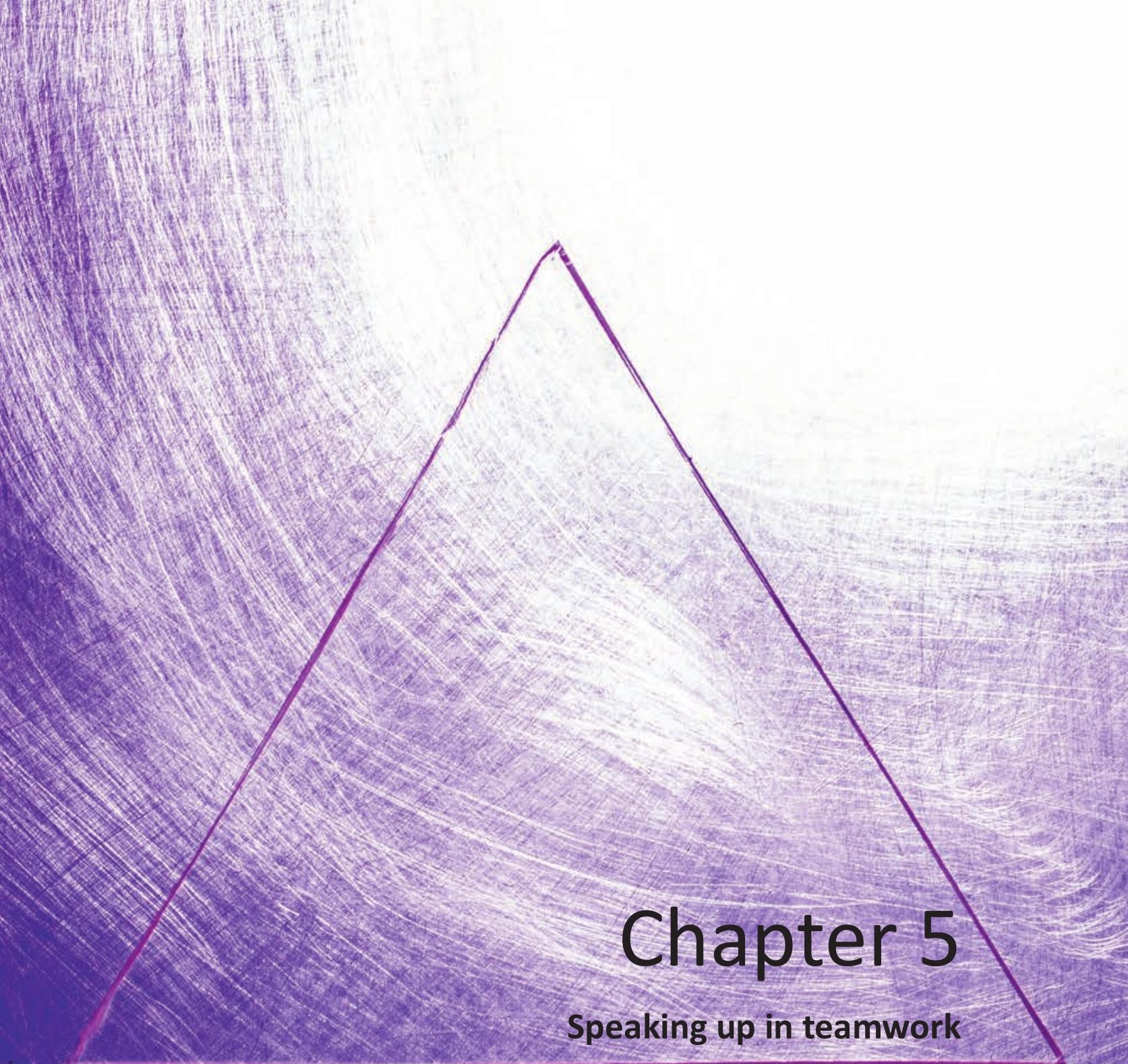

Speaking up in teamwork

Speaking Up among Clinical Teachers in Postgraduate Medical Training.

(Submitted Advances in Health Sciences Education)

Irene Slootweg, Albert Scherpbier, Renée van der Leeuw,

Maas Jan Heineman, Cees van der Vleuten, Kiki Lombarts 


\begin{abstract}
The importance of speaking up for safeguarding quality of patient care is increasingly being endorsed in research findings. However, little is known about speaking up of clinical teachers in postgraduate medical training. In order to determine how clinical teachers demonstrate speaking up in formal teaching team meetings and what factors influence this, the authors carried out an exploratory study based on ethnographic principles.The authors selected 12 teaching teams and observed, audio recorded and analysed the teaching team meetings. Subsequently, during an interview, the program directors reflected on speaking up of those present during the meeting. Finally the authors analysed all data iterative, using a template analysis, based on Edmondson's six behaviors of speaking up.The study conducted from October 2013 to July 2014 and ten teaching team participated. During the teaching team meetings, the clinical teachers exhibited most of the behaviors of speaking up. 'Sharing information' strongly resembles providing information and 'Talking about mistakes' occurs in a general sense and without commitment of improvement activities. 'Questions were asked'; often closed and at times several questions simultaneously. The authors identified factors that influence speaking up by clinical teachers: relational, cultural, and professional. The clinical teachers exhibit speaking up, but there is only limited awareness to discuss problems and mistakes. The way clinical teachers discuss mistakes, centred mainly on the question of blame. It is important to take into account the factors that influence speaking up, in order to stimulate open communication during the teaching team meetings.
\end{abstract}




\section{Introduction}

In order to meet the needs of postgraduate medical training, clinical teachers devote increasing time to teamwork. ${ }^{1-3}$ Teamwork is required to discuss and agree upon a range of issues, including: 1) implementation of the training requirements, 2) supervision of the residents, 3) assessment of the residents' performance, and 4) safeguarding of patient safety. ${ }^{4,5}$ Research on the functioning of teams has been carried out by Edmondson, who introduced the term 'teaming' in her work on high performance teams. ${ }^{5}$ She describes teaming as 'a dynamic activity based on members who have teamwork skills and are therefore able to be flexible in working together irrespective of when, where or with whom'. Teaming is characterised by a number of different behaviors, one of which is speaking up. Speaking up is defined as a sincere and direct manner of communication between individuals, including asking questions, seeking feedback and discussing mistakes, and has been shown to have a preventive effect on human error. ${ }^{5}$ Not speaking up, for example as a result of anxiety or from a desire to avoid conveying bad news or unwelcome ideas, may protect the individual, but is detrimental for the performance of the team. ${ }^{4}$ From the literature it is also known that communication and specific sharing information is essential for learning from mistakes, for both individuals, teams and organizations. ${ }^{6-8}$ We are interested in the way in which clinical teachers apply speaking up behaviors and talk about problems and mistakes. From an earlier study we became aware that the relational communication of clinical teachers is not always their strength and teamwork for postgraduate medical training is not self-evident. ${ }^{9}$ What is still unknown is how speaking up is displayed and interpreted by clinical teachers in the context of their discipline-specific meetings and discussions. This qualitative study is intended to make a contribution to understand teamwork among clinical teachers, based on the research question: How do clinical teachers demonstrate the behaviors of speaking up, and what are the factors that influence these behaviors? This knowledge can help with developing interventions to promote speaking up within the teamwork of clinical teachers. We focus particularly on the formal discipline-specific teaching team meetings and how the program directors view the speaking up behaviors, displayed during these meetings. Our aim is to make a contribution to broadening the knowledge about speaking up by healthcare professionals. 


\section{Methodology}

The study was carried out based on a constructivist paradigm. It focuses on participants' practices in which participants' accounts are treated as narrative accomplishments rather than as true or false reports of reality. ${ }^{10,11}$ As teamwork is complex and calls for solid research, and as much of the existing research on teamwork does not fully reflect this complexity, we combined observation and interviews based on ethnographic principles. ${ }^{10,12-14}$ Classical ethnography assumes a long-term engagement in a study setting and the collection, through observation and conversational interviews, of data that are analysed to understand the meaning inherent in the everyday activities of a particular group. ${ }^{15}$ Inspired by this, we opted in this study to observe social interactions that occur within a group, at a particular point in time, namely during formal teaching team meetings. We subsequently supplemented these observations by conducting interviews with the program director, who were also chairing the teaching team meetings in most cases. ${ }^{11}$ The program director has an overview of the collective and has to monitor and promote effective teamwork, which includes encouraging speaking up among clinical teachers.

\section{Setting and participants}

The study was carried out in the Netherlands where residency training programs are coordinated and delivered by University Medical Centers and in regional affiliated teaching hospitals, where residents work alongside and are supervised by clinical teachers. Program directors have the formal hierarchical position of head of the clinical teaching team, a task for which they are mostly highly motivated. ${ }^{16}$ In the Dutch context, it is compulsory that at least four formal meetings on postgraduate medical training are held annually. The meetings are attended by clinical teachers (TC) and the program director (PD); residents may or may not be in attendance. For this explorative study we used purposeful sampling to select 12 teaching teams with as diverse as possible range of specialist fields, group sizes and hospitals. ${ }^{17}$ The invitations were sent by mail, addressed to the program director. Informed consent was checked at three instances: the program director was asked by email; participants were asked verbally at the start of the meeting; and the program director was asked by means of 
a form at the start of the interview. The Ethical Review Board of the Academic Medical Center (the university medical center associated with the University of Amsterdam) waived ethical approval for this study.

\section{Process of data collection and analyses}

We started data collection by attending one teaching team meeting for each group and making observations on the basis of the six behaviors of speaking up outlined by Edmondson: 1) asking questions, 2) sharing information, 3) seeking help, 4) experiment with unproven actions, 5) talking about one's own mistakes and 6) seeking feedback. For each of the meetings, one observer was present (IAS or RvdL) who worked as unobtrusively as possible. ${ }^{18}$ The observations were noted in writing with comments added, and audio recordings were made. The notes and the recordings were coded by two researchers (IAS and RvdL) and were discussed until agreement was reached. The interviews with the program director took place within one month after the observations and following the first analysis of the audio fragments from the meeting. The interviews were held according to protocol, based on specific audio fragments. The program director was asked after each fragment to reflect on the speaking up during the meetings, using a three-point scale: 1) quite limited communication, 2) respectful but guarded communication, and 3) open reciprocal communication. ${ }^{5}$ The interviews were all conducted by the first author (IAS) and were recorded. ${ }^{19}$

All the data collected from observations and interviews were iteratively analysed using a template analysis based on the six behaviors of speaking up. ${ }^{20}$ The first step was to analyse the observations, whereby audio fragments were coded using the software package Atlas T. In this process, fragments were selected that were later used for the interviews. ${ }^{21,22}$ The choice of fragments was based on a range of different features: all fragments related to a team interaction and illustrated one of the six behaviors of speaking up. The fragments were on average three minutes long. The second step was to analyse the interview recordings. The sound fragments were also coded using Atlas $\mathrm{T}$, in the template where the data from the observation was analysed. See Box 1 for a step-by-step process description. 


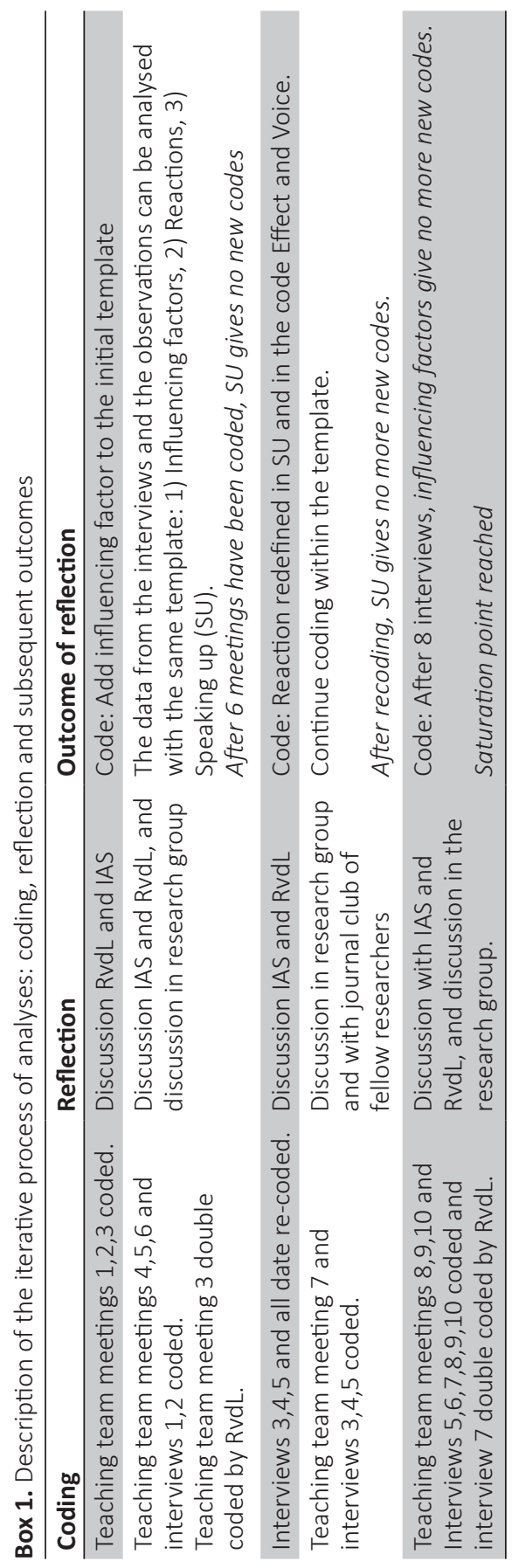




\section{Results}

The data collection and analysis took place in the period from October 2013 to July 2014. Of the 12 teaching teams approached, two did not accept the invitation; one because of the timing of the study and one without indicating the reason: four from non-surgical specialties, four from surgical specialties and two from the supporting disciplines. The teams were from University Medical Centers (3) and teaching hospitals (7). The team size varied from 7 to 30 clinical teachers. On average there were seven clinical teachers and six residents present during the teaching team meetings. The agenda items varied from teaching activities to the assignment of tasks, timetables, legislation, regulations and social activities (Box 2). One of the audio recordings of an observed team meeting failed for technical reasons. The subsequent interview with the program director was therefore conducted on the basis of the notes taken by the observer.

Box 2. List of discussion topics

- $\quad$ Teaching activity and trajectory

- $\quad$ Feedback \& Portfolio

- Faculty development of clinical teachers

- Leadership of program director

- Introduction program of the residents

- Deviding teaching tasks

- Timetables of the residents

- Quality Improvement Activity

- Students of Bachelor \& Masters Medicine

- Accreditation and Law from National Board

- $\quad$ Teambuilding \& Social events

\section{Speaking Up}

The results relating to the first research question address the way in which the clinical teachers demonstrated speaking up. We describe successively the six behaviors of speaking up.

The behavior that clearly manifested itself during the teaching team meetings was talking about mistakes, only not one's own mistakes. During the meeting, mistakes 
and problems were discussed mainly in a general sense, whereby statements were exchanged and consensus was sought about problems. Conclusions were not drawn or there was evident jumping to conclusions. If clinical teachers talked about undesirable behavior, it was not always done directly or clearly. The behavior of asking questions was also clearly evident during the meetings. Varying types of questions were asked, both open and, more often closed. At times several questions were asked simultaneously, and people did not wait for the answer. Much of the teaching team meeting time was taken up with the behavior of sharing information. Information was given about best practice, previously made agreements and teaching requirements. We observed that during the meeting colleagues regularly shared information with their neighbours in private asides rather than with the team as a whole. Some teaching team meetings consisted wholly of giving information. In these meetings it was the program director who spoke most of the time. The seeking help behavior consisted of the program director and clinical teachers asking for assistance in implementing teaching policies. This behavior proceeded relatively automatically and was almost always responded to positively. Program Director (PD) 3 during the interview: 'We ask one another for help, including about teaching matters. It's something we are also used to doing with second opinions in patient care.' The seeking feedback behavior was expressed by asking for feedback about established policy or about personal performance. In both cases it was the program director who set an example and asked for feedback. No requests for feedback among clinical teachers were observed. The behavior of experiment with unproven actions was scarcely observed. One of the program directors (PD 4) gave his reaction to this: 'We are a cautious team: afraid of new initiatives and particularly good at following rules.'

Summarizing, four of the six behaviors of speaking up were clearly observed during the teaching team meetings. Sharing information primarily takes the form of providing information. Talking about mistakes was mainly expressed in a general sense and does not always lead to a result. Closed questions were often asked, but without waiting for the answer, or no answer came forth. Clinical teachers generally asked one another for assistance. Only the program director asked for feedback, and this only minimally, on issues of policy or about his/her performance as leader of the program. Unproven actions were almost never tried out. 


\section{Influencing factors}

The results relating to the second research question address the influencing factors of speaking up by clinical teachers. We identified three factors that influence speaking up mainly through the interviews with the program directors and their reflections on the speaking up fragments. (Box 3)

Box 3. Influencing factors on speaking up of clinical teachers during teaching team meetings

\begin{tabular}{|c|c|c|}
\hline \multicolumn{3}{|c|}{ Influencing factors on speaking up of clinical teachers during teaching team meetings } \\
\hline \multirow[t]{4}{*}{$\begin{array}{l}\text { Relational } \\
\text { Factors }\end{array}$} & Power & $\begin{array}{l}\text { PD 1: 'Sometimes there is a lack of dignity. In the meeting } \\
\text { people just want to show who's the boss'. }\end{array}$ \\
\hline & Leadership & $\begin{array}{l}\text { PD 9: What they say is: 'Medical training is your thing so } \\
\text { you should look after that. [.....] Let's not spend too much } \\
\text { time on it'. }\end{array}$ \\
\hline & Feeling Safe & $\begin{array}{l}\text { CT } 2 \text { during the meeting: 'Isn't this [proposal] rather } \\
\text { unexpected?' PD: 'No, we are a flexible group'. CT: 'Yes, } \\
\text { I know that you are flexible, but might it be an idea to } \\
\text { brainstorm on this?' }\end{array}$ \\
\hline & Handling Conflict & $\begin{array}{l}\text { PD } 3 \text { during the meeting when taking his team to task for } \\
\text { undesirable behavior: 'Anyone who blushes knows things } \\
\text { should be different.' }\end{array}$ \\
\hline \multirow[t]{3}{*}{$\begin{array}{l}\text { Cultural } \\
\text { Factors }\end{array}$} & History & $\begin{array}{l}\text { PD 8: 'We have a long history and there's a lot of going over } \\
\text { old ground'. }\end{array}$ \\
\hline & Meeting Culture & $\begin{array}{l}\text { PD 7: 'The core [of the staff] know what we think without } \\
\text { having to say it'. }\end{array}$ \\
\hline & Feedback Culture & $\begin{array}{l}\text { PD 3: 'We are all able to say anything to one another: there } \\
\text { may be harsh words, but they're soon followed by a pat on } \\
\text { the back'. }\end{array}$ \\
\hline \multirow[t]{4}{*}{$\begin{array}{l}\text { Professional } \\
\text { Factors }\end{array}$} & $\begin{array}{l}\text { Commitment to } \\
\text { Teaching }\end{array}$ & $\begin{array}{l}\text { PD 6: 'This is about teaching, which is something everyone } \\
\text { enjoys. Meetings about money are much tougher'. }\end{array}$ \\
\hline & $\begin{array}{l}\text { Contribution by } \\
\text { Residents }\end{array}$ & $\begin{array}{l}\text { PD 8: 'The residents don't say 'yes' and that's the end of it. } \\
\text { They really dare to say what they think'. }\end{array}$ \\
\hline & Discipline-specific & $\begin{array}{l}\text { PD 9: 'It's typical of anaesthetists to give an OK, shut the } \\
\text { door and then do whatever they want'. }\end{array}$ \\
\hline & Personality traits & PD 8: 'He determines the way we work together'. \\
\hline
\end{tabular}

\section{1) Relational Factors}

Speaking up is influenced by the extent to which there is a power struggle between the program director, the deputy director and the head of the medical department. Hierarchical relations also played a role here, for example, as one program director 
indicated (PD 2): 'You can't force people and give them orders'. This power struggle generated an atmosphere, with team members interrupting one another and talking among themselves, causing clinical teachers to drop out. Hardly any questions were asked, there was no drilling down on questions and talking about mistakes mainly centred on the question of blame. The program directors made it clear that they were aware of their leadership position as role models for speaking up during the meetings. They indicated that they felt responsible for the teaching and for the well-running of the meetings. They struggled with encouraging others to speak up and seemed mainly to look for ways of sharing information and therefore responsibility. For the program directors it became also clear that feeling safe influenced speaking up among clinical teachers. They reflected on examples of questions being asked cautiously, verbosity, talking over one another, contradicting one another and people speaking in defensive, reproachful tones. Handling conflict behavior on the part of the clinical teachers primarily takes the form of safeguarding harmony. The program directors indicated this as a reason during the interviews and stressed the importance of 'wanting to be perceived as being nice'. It was noticeable that conflict avoidance behavior was common in discussing problems without drawing a conclusion, or conversely by jumping to conclusions, ridiculing and not responding to questions.

\section{2) Cultural Factors}

The significance of past events was clearly evident during the whole of the data collection. Some teaching teams were typified by the program director reproaches, mutual dissatisfaction and disagreements. PD 2: 'Having undergone mediation, our team is damaged. The atmosphere has become embittered'. In these teams, speaking up primarily consisted of laying down information and asking - mainly closed questions. The converse was also true. In a team that had worked together for a long time, often under the same team leader, the members knew what they could expect of one another, and the program director was able to create an atmosphere of safety. Some teaching team meetings proceeded in a business-like and efficient meeting

culture. There was an agenda, that seemed to be well-prepared and this resulted in little speaking up during the meeting. There were also teaching team meetings that ran over time, where there was a lack of structure and people frequently talked over 
one another and failed to listen. Finally, it was apparent that situations that occurred in the department were not always evident in the meetings. In many cases, according to the program director, the real problems were not discussed in the meetings, but in the corridors or in the staff meetings without the residents. Also, it became clear that clinical teachers handle feedback differently. There were teams that were characterised by the program director as anxious, where the clinical teachers were not conducive to giving feedback, while there were also teams where giving feedback, even unsolicited feedback, was appropriate for the style of interaction. Some teams did little with feedback, giving the desire for harmony as the reason.

\section{3) Professional Factors}

The teaching requirements, whether or not bureaucratically imposed, determined the degree to which clinical teachers demonstrated speaking up. There were teaching teams that held meetings because it was required; because the assessment audits required it of them. In the meetings, information was given, not shared, and problems were discussed from the viewpoint of concerns and difficulties. In that meeting fewer clinical teachers were present and the attention of those who were there was frequently elsewhere. According to the program directors, this behavior was also evident among clinical teachers for whom teaching, and in particular modern teaching programs, had little priority. If residents were present at the meetings, they played different roles. They acted as chairman, made proposals, spoke openly about problems, demonstrated strength as a group or as individuals. If the program director had prepared the meeting thoroughly with the residents, there was little speaking up and the meeting was more like an information session. During the interviews the program directors also talked about the discipline-specific nature of the specialism and speaking up. The non-surgical specialties showed they were prepared to reflect, while the surgical specialists did not consider themselves meeting types and felt that they were people of few words. The program directors of the supporting specialists suggested that communication was not always seen as a strong point of their specialism.

We observed that every team had at least one conspicuous colleague who influenced with his personality traits the speaking up within the team. We heard about the rebel, 
who was against everything. We heard about the clinical teacher who was known to be an excellent doctor, who spoke reservedly and, if what he was saying was not clear, carried on regardless. We heard about the clinical teachers who did not feel accepted in their position and who expressed their thoughts strongly in the meetings. The program directors spoke during the interviews about the negative attention that these people not only demanded in the group but also received.

Summarising, three factors were found that explain speaking up. The relational, cultural and professional factors influence the way in which clinical teachers collaborate during teaching team meetings for the benefit of the quality of postgraduate medical training.

\section{Discussion}

This study suggests that in terms of the six behaviors of speaking up teaching teams seem to discuss mistakes and problems, although superficially and almost never personally. These discussions rarely lead to concrete improvement actions. Also, we learn that in the teaching team meetings questions are asked, but that the answers are less important, and that information is given, but that this often meets with little or no response. The reported findings resonate with the literature on team interaction, reporting that there are 'elephants in the room': issues that seem to be impossible to discuss., ${ }^{7,23}$ We all see the 'elephant', it gets in our way, but we lack the will, the courage or the skills to discuss problems and mistakes that are quite obvious, or to confront one another with them. ${ }^{23}$ We may therefore not be adequately equipped to resolve mistakes and problems effectively. The price of not speaking up is that the organization does not learn from mistakes. ${ }^{7,23}$ The fact that in the teaching team meeting the clinical teachers potentially not learn adequately from mistakes, may have an impact on the quality of postgraduate medical training. It is likely that clinical teachers could supervise residents better if they themselves knew what it is like to learn from mistakes. 
There appear to be three factors that influence speaking up and that can break through or maintain the silence in the teaching team meetings. Clinical teachers are more inclined to avoid conflicts than to discuss them openly. We also see this aspect in the study relating to the question of 'What is happening below the surface'. ${ }^{24}$ This study gives an indication that power and conflict have an influence on interpersonal behavior, with the result that three types of conflicts are inadequately discussed. First there are the task-related conflicts, about people's ideas on and opinions of tasks. In the teaching team meetings we saw examples of discussing the compulsory teaching tasks and discussions on opinions of modernization of the postgraduate medical training. Differences of opinion among the clinical teachers remained undiscussed: the 'elephant' that everyone avoided talking about.7,23 Besides the task-related conflicts, there are also the individual clashes about incompatibilities relating to personal issues. The professional factors, with the personality traits both seem to influence speaking up. Research by Keyton on interaction in dysfunctional teams shows that in every team there are 'provokers' and that the team leaders believe that if this provoker is removed or fixed, the team will function more effectively and efficiently. ${ }^{25}$ What will help here is an open reciprocal communication and conflict management. ${ }^{25}$ Thirdly, Janss indicates that there are process conflicts that refer to logistical or delegational issues, such as responsibilities. ${ }^{24}$ The relational factors and in particular leadership seem to be important in encouraging clinical teachers to take responsibility for teaching and to practice speaking up. ${ }^{7}$ From the study on the willingness in surgeon behavior to speak up about training, it is known that the senior surgeon does indeed have an important role in improving the communication between junior and senior clinicians to enhance patient safety. ${ }^{26}$

The cultural factor seems to add significance to the results of speaking up. Here factors are ignored that have played a role for some time, as deep-rooted values, norms and habits. One example is experimenting with new behavior that scarcely features at all. Edmondson shows how important an atmosphere of safety is for the learning process of collective discussions and experimenting with new behaviors. Safety, as we indicated earlier, is not always felt in teaching teams. ${ }^{4,5,27}$ 
The strength of this study is in the use of both observations and interviews. The data from the interviews has reinforced the data from the observations and added to its significance. We analysed the audio fragments rather than taking the spoken text verbatim, which generated a thorough analysis. The six behaviors of speaking up give a clear focus to the data and make an accurate contribution to providing an answer to the research questions. The limitation of this study is in the assumption that one meeting is representative for the team interaction among clinical teachers. For this reason, future research should focus on the team interaction on postgraduate medical training in daily practice. A further limitation is the fact that this study was carried out in the Netherlands where a culture of open feedback may be more acceptant than in other cultures. International comparative research in the area of speaking up would be interesting.

\section{Practical implication and future research}

The results of this study can be used as a source of inspiration for program directors to improve speaking up as a shared learning process that could help clinical teachers in their collaborative task of training residents. Follow-up research should focus on a more longitudinal study and more thoroughly observing team interaction among clinical teachers to investigate which influencing factor is most defining for whom and for which specialty. Further, now that we know more about the formal teaching team meeting, it would be interesting to analyse the way that mistakes in postgraduate medical training are discussed by clinical teachers in an informal setting.

\section{Conclusion}

Clinical teachers demonstrate behaviors of speaking up during teaching team meetings, whereby it appears that the problematic topics are only discussed to a limited degree. Mistakes and conflicts are mainly discussed in a general sense, and are often neither directed at the individual nor adequately result-oriented, which means that the 
positive effect that speaking up could have on the quality of postgraduate medical training is largely lacking. If clinical teachers are to develop the behaviors of speaking up, it is important to take into account the relational, cultural and professional factors that influence speaking up in order to stimulate sincere and direct communication during the teaching team meetings. 


\section{References}

1. Eva KW. (2002) Teamwork during education: the whole is not always greater than the sum of the parts. Medical education, 36(4), 314-316.

2. Frank JR, Snell LS, Cate OT, et al. (2010) Competency-based medical education: theory to practice. Medical teacher, 32(8), 638-645.

3. Harden RM. (2011) Looking back to the future: a message for a new generation of medical educators. Medical education, 45(8), 777-784.

4. Edmondson AC. (2003) Speaking up in the operating room: How team leaders promote learning in interdisciplinary action teams. J Manage Stud, 40(6), 1419-1452.

5. Edmondson AC. (2012)Teaming: How organizations learn, innovate, and compete in the knowledge economy. San Francisco, John Wiley \& Sons.

6. Okuyama A, Wagner C, Bijnen B. (2014) Speaking up for patient safety by hospital-based health care professionals: a literature review. Bmc Health Serv Res, 14.1,61.

7. Dankoski ME, Bickel J, Gusic ME. (2014) Discussing the undiscussable with the powerful: why and how faculty must learn to counteract organizational silence. Academic medicine : journal of the Association of American Medical Colleges, 89(12), 1610-1613.

8. Bleakley A, Allard J, Hobbs A. (2013) 'Achieving ensemble': communication in orthopaedic surgical teams and the development of situation awareness-an observational study using live videotaped examples. Advances in health sciences education : theory and practice, 18(1), 33-56.

9. Slootweg I, Lombarts K, Van Der Vleuten C, Mann K, Jacobs J, Scherpbier A. (2013) Clinical teachers' views on how teaching teams deliver and manage residency training. Medical teacher, 35(1), 46-52.

10. Silverman D. (2011) Interpreting qualitative data. Sage.

11. Swanwick T. (2011) Understanding medical education: Evidence, theory and practice. San Francisco, John Wiley \& Sons.

12. Lingard L, McDougall A, Levstik M, Chandok N, Spafford MM, Schryer C. (2012) Representing complexity well: a story about teamwork, with implications for how we teach collaboration. Medical education, 46(9), 869-877.

13. Stewart GL. (2010) The Past Twenty Years: Teams Research Is Alive and Well at the Journal of Management. J Manage, 36(4), 801-805.

14. Atkinson P, Pugsley L. (2005) Making sense of ethnography and medical education. Medical education, 39(2), 228-234.

15. Bosk CL. (2003) Forgive and remember: managing medical failure. Chicago, University of Chicago Press.

16. Slootweg IA, van der Vleuten C, Heineman MJ, Scherpbier A, Lombarts KM. (2014) Program directors in their role as leaders of teaching teams in residency training. Medical teacher, 36(12), 1073-1079.

17. Pope C, van Royen P, Baker R. (2002) Qualitative methods in research on healthcare quality. Quality \& safety in health care, 11(2), 148-152.

18. Franz TM. (2012)Group dynamics and team interventions: understanding and improving team performance. San Francisco, John Wiley \& Sons.

19. Lyle J. (2003) Stimulated recall: a report on its use in naturalistic research. Brit Educ Res J, 29(6), 861-878.

20. King N, Cassell C, Symon G. (2004) Using templates in the thematic analysis of texts. Essential guide to qualitative methods in organizational research, 256-270.

21. Ringsted C, Hodges B, Scherpbier A. (2011) 'The research compass': an introduction to research in medical education: AMEE Guide no. 56. Medical teacher, 33(9), 695-709. 
22. Tavakol M, Sandars J. (2014) Quantitative and qualitative methods in medical education research: AMEE Guide No 90: Part I. Medical teacher, 36(9), 746-756.

23. Souba W, Way D, Lucey C, Sedmak D, Notestine M. (2011) Elephants in academic medicine. Academic medicine : journal of the Association of American Medical Colleges, 86(12), 1492-1499.

24. Janss R, Rispens S, Segers M, Jehn KA. (2012) What is happening under the surface? Power, conflict and the performance of medical teams. Medical education, 46(9), 838849.

25. Keyton J. (1999) Analyzing interaction patterns in dysfunctional teams. Small Gr Res, 30(4), 491-518.

26. Barzallo Salazar MJ, Minkoff H, Bayya J, et al. (2014) Influence of surgeon behavior on trainee willingness to speak up: a randomized controlled trial. Journal of the American College of Surgeons, 219(5), 1001-1007.

27. Edmondson AC. (1999) Psychological safety and learning behavior in work teams. Administrative Science Quarterly, 44(2), 350-383. 



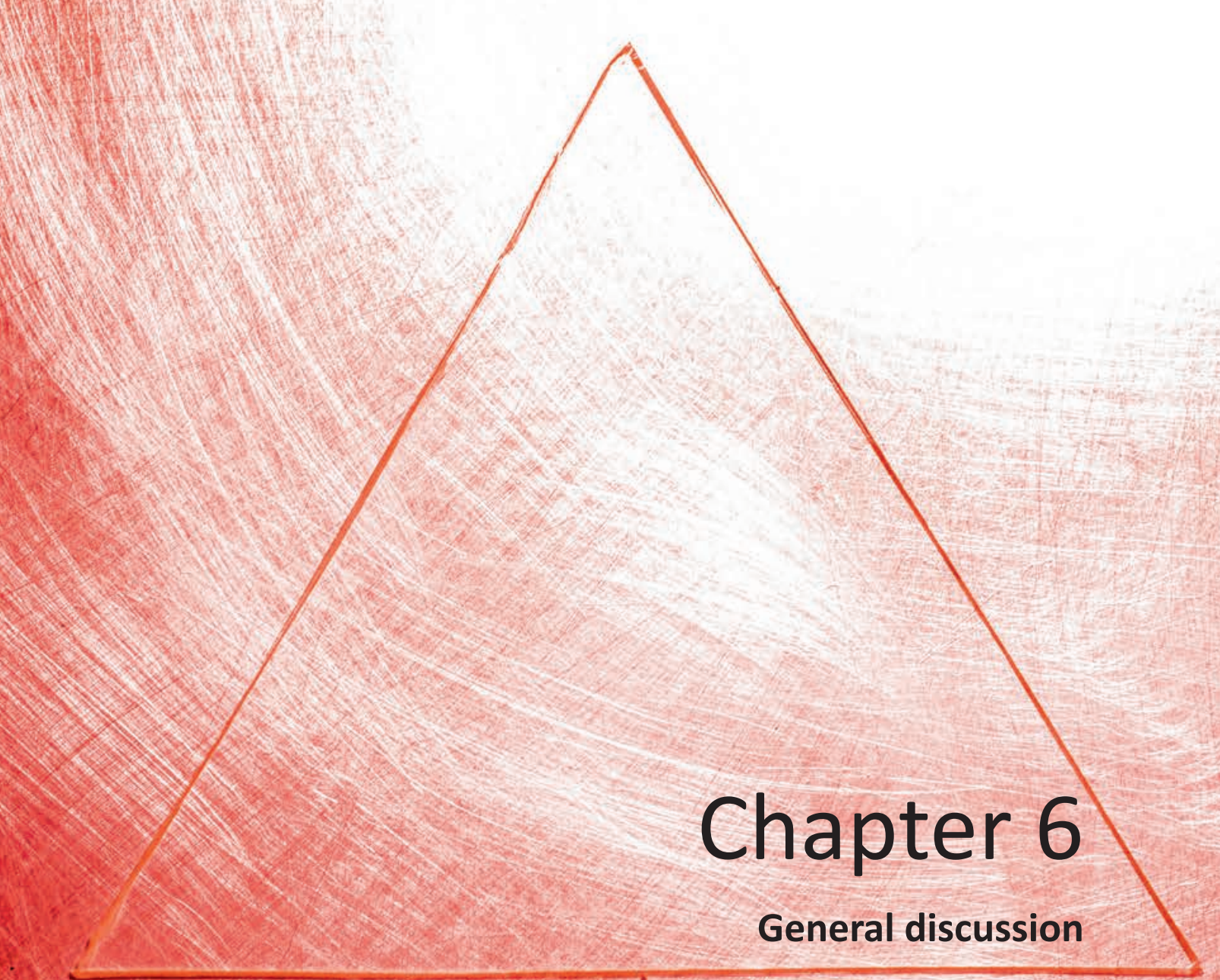


106 | Chapter 6 
This chapter discusses the main findings of our four studies by answering the overall research questions: What is the nature of teamwork among clinical teachers? and What is the role of leadership in supporting the teamwork of clinical teachers? The aim is to deconstruct the teamwork of clinical teachers in the field of postgraduate medical education. We provide an answer to the research questions based on insights from medical professionalism, social cultural learning theory and organizational studies. This chapter is based on the four main findings: (1) the clinical teacher as medical professional, (2) the action process as key team process, (3) the joint team result, and (4) leadership focused on teaching tasks. This thesis concludes with the implications for future research and for the practice of medical education.

\section{The clinical teacher as medical professional}

'As long as the other team member has the right team spirit...'. (Quote clinical teacher)

This is a quote by one of the clinical teachers participating in our studies. Clearly, teamwork is not just a matter of the right team spirit of one of the team members. Practicing and developing individual teamwork qualities by all members of a team, along with working towards a shared goal, are widely regarded as the core of teamwork. ${ }^{1}$ Individual teamwork qualities refer to the knowledge, skills and attitudes that clinical teachers need in order to be able to work together effectively. This means, for example, having a good understanding of the vision, aims and joint tasks of a teaching team. It also means possessing the skills that are needed for good teamwork, such as adaptability, performance monitoring and feedback, communication, conflict management and decision-making. The attitudinal qualities that are important for teamwork include collective efficacy, shared vision, mutual trust and the belief in the importance of teamwork in postgraduate medical training. ${ }^{2,3}$ From the different studies described in this thesis it is clear that clinical teachers pay little or no attention to practicing and developing individual teamwork qualities. This was seen in the understanding of teamwork, the skills of giving and receiving feedback, managing conflict, and decision-making, as well as in the belief of teamwork in postgraduate medical training. 
In the first study, investigating the experiences of the clinical teachers with teamwork, it became apparent that the clinical teachers were more passionate about clinical expertise than about specific knowledge of teaching and teamwork. A shared educational vision and goals were considered to be of relatively little importance. The study also showed that the teamwork qualities of giving and receiving feedback were difficult for clinical teachers. This was further observed during the study of the evaluation instrument (TeamQ), which examined how individual members of teaching teams evaluate their current levels of teamwork. In this study feedback culture was shown to score lowest in the opinion of the clinical teachers. The importance of giving and receiving feedback as a teamwork quality has been underpinned in various organizational studies, which endorse feedback as a key element of teamwork. ${ }^{4,5}$ Feedback enables a team to obtain information about the quality and quantity of its team results, as well as knowledge about the effectiveness of the methods used to achieve the desired levels of team performance.

The study on team interaction between clinical teachers showed that resolving problems, a teamwork skill, was displayed in such a way that there was only limited discussion of problem issues. Mistakes were mainly discussed in a general sense, and were often neither directed at the individual nor sufficiently result-oriented. In terms of handling conflicts, another important teamwork quality, it appeared that among clinical teachers the prime concern when handling conflicts is to safeguard harmony. It was evident that conflict avoidance behavior was common among clinical teachers when discussing problem issues, and that consequently in many discussions no conclusions were drawn, or conversely team members would jump to conclusions, ridicule other people or not respond to questions.

It is important that teamwork qualities are practiced and developed in order to achieve open and direct team interaction among clinical teachers. The team interaction study showed that the medical profession, with its discipline-specific character, is one of the factors that influence team interaction: 'We surgeons are people of few words, and 'We internal medical specialists are observers. Everyone gives issues a lot of careful thought, are mild and open to feedback'. To be able to work in teams it is important to take into account the individual behaviors of the autonomous professional: altruism, accountability, respect and integrity. ${ }^{6}$ In conclusion, we could say that in this thesis 
there is little or no evidence of teamwork qualities being practiced and developed by clinical teachers in clinical teaching teams.

\section{The action process as key team process}

'The way we all sit there together .... We don't make too much of things..... Let's stay relaxed; We've spent the whole day working' (Quote from program director)

This quote is typical of the comments that clinical teachers or program directors made about the team process of working together in clinical teaching teams. We recognize in these comments the view that the team process is frequently considered to be a black box of teamwork, because it is unclear what really happens when a team member with the right teamwork qualities does not achieve the right team results. ${ }^{2,7}$ The team process connects team members' individual teamwork qualities with the team results and can be divided into three processes: (1) the transition process, which consists of mission analysis and formulating goals and strategy, (2) the action process, comprising task accomplishment, coordination and monitoring progress, and (3) the interpersonal process, referring to conflict management, motivation and confidence building. ${ }^{7}$ The different studies on which this thesis is based make it clear that the team process is primarily an action process, and that clinical teachers pay little or no attention to the transition process and the interpersonal process. The action process, something that clinical teachers consider important, was primarily focused on implementing teaching tasks, but results in hardly any concrete agreements about the training of residents, the monitoring of the progress of team results and giving one another feedback. If any interpersonal processes do occur, these seem to be aimed specifically at avoiding conflicts and preserving harmonious relations. The studies show little or no evidence of the transition process, where the focus is on the mission statement and strategic goals.

The conclusions about the team process are supported firstly by the study on the experiences of clinical teachers with teamwork, in which it was made clear that the discussions among them were mainly concerned with residents' performance and 
the division of teaching tasks. Postgraduate medical training was mostly coordinated in line with a well-established hierarchical structure that determined the division of teaching tasks and responsibilities; the so-called action processes.

Secondly, an important finding about the team process emerged from the study of the evaluation instrument (TeamQ). This also showed that clinical teachers consider the team process important. In the evaluation instrument this team process is divided into two themes, namely processes relating to the feedback culture and decision-making. These two action processes are particularly important in monitoring the progress of the training of residents.

The study on the team interaction between clinical teachers concentrated specifically on interaction as a team process and the factors influencing this. Firstly, there were the cultural factors that match the meeting traditions of the clinical teachers. During meetings, mistakes and problems were discussed primarily in a general sense, whereby statements were exchanged and consensus was sought about the problems. Either conclusions were not drawn or there was evident jumping to conclusions, ridiculing people and not responding to questions. Secondly, the relational factors such as feeling safe, power, leadership and handling conflicts played a role in the team process. It was noticeable that conflict avoidance behavior was common in discussing problems amongst clinical teachers in the formal meetings. Thirdly, professional factors appeared to influence the team process: 'Saying and doing are two different things for us. We mean well and say that we definitely have to give one another feedback, but if it doesn't suit us, we ignore it.' This is in line with the research on the way that medical professionals are used to dealing with one another. ${ }^{8}$ This research distinguishes three types of behavior, all three of which are recognizable in the team process between clinical teachers: (1) not giving orders, not controlling each other, and consensual decision-making, (2) being a good colleague, doing each other favors and being loyal to each other (3) not openly criticizing each other. Criticism is mostly disguised by posing questions. ${ }^{8}$ The literature on team effectiveness, defined as a manager's or customer's judgment about the acceptability of team results, ${ }^{2}$ also clearly shows the importance of participation and cohesion as positive influencing factors for the team process. ${ }^{9}$ Finally, social cultural learning theory stresses the importance of converting mutual engagement into making and adhering to concrete agreements. ${ }^{10-12}$ Paying 
lip service to participation and engagement is not enough; concrete agreements between team members in teamwork are crucial. ${ }^{11}$

In conclusion we could say that this thesis has somewhat lifted the lid on the black box of teamwork between clinical teachers. Our studies strongly suggest that the team process primarily constitutes the action process and that little use is made by clinical teachers of the transition process or the interpersonal process.

\section{The joint team result}

'I believe it is important to only show the beautiful aspects of our specialty'

(Quote from clinical teacher)

This quote exemplifies the passion of a clinical teacher who trains residents as a professional in his or her own way. We may conclude in this thesis that the various studies conducted produced insufficient evidence of a joint team result of the clinical teachers together.

Firstly, this became evident in the study that investigated the experience of the clinical teachers with teamwork, and from which we learned that clinical teachers appeared to focus mainly on individual effectiveness rather than on team effectiveness. It was clear that the clinical teachers did not use teamwork as an intentional organizational strategy to optimize the quality of residency training. Clinical teachers seem to have little awareness of the importance of a team result and do not appear to work with this goal in mind. A shared educational vision and goals were considered to be of relative importance. This is also in line with the findings from the leadership study. We were not able to determine from this study whether and to what extent the program director manages for results, although we do know that team leadership behaviors are important to achieve team results. ${ }^{13}$

Another finding arose from the evaluation instrument study (TeamQ) and made clear that measuring team results is challenging for the evaluation of teamwork in teaching teams. The TeamQ evaluation instrument comprised one specific theme about the joint team result and two themes about supervising and assessing the residents. From the literature we know that the results of teamwork are not always clearly defined 
in the minds of the team members. ${ }^{9}$ There is a relationship between team process behavior and the team results; however, in many cases the quality of the relationship is not clear, mainly because the results of teamwork are often vague and can be different for individual team members. ${ }^{14-16}$

In conclusion we can say that the team results as such reflect the type of teamwork that clinical teachers want to strive for, but that, in terms of the nature of teamwork, the visible team results of clinical teaching teams are insufficiently demonstrated in this thesis.

\section{Leadership focused on teaching tasks}

'My principle is that we have to have the best training program in the hospital.

I am number 1.' (Quote from program director)

This quote exemplifies the strength of the individual ambition of a program director. The question is to what extent this program director has invested in making this ambition a collective goal. We were able to determine from the different studies that the program directors focus on carrying out the teaching tasks and, because less attention is paid to investing in collectivity, they support teamwork in clinical teaching teams only to a limited degree. The different studies in this thesis give an insight into clinical teachers who indicate that they need a leader who acts as a kind of 'superclinical teacher' and who takes on the lion's share of the teaching tasks. The program director can be seen as a role model in carrying out teaching tasks and is trusted and valued as such by the clinical teachers. However, the program director does not seem work on developing collective ambitions and goals, but is primarily task oriented. ${ }^{7}$ This largely explains the limited support for teamwork.

The first finding of the study on the experience of clinical teachers with teamwork was that the lack of clarity with regard to the program director's role as team leader contributed to the lack of coherence in teams of clinical teachers. The program directors felt responsible for the quality of residency training and for fostering a sense of joint responsibility within their teaching team. They tried to divide teaching tasks among team members and additionally performed many basic teaching tasks themselves. 
The second finding came from the evaluation instrument study (TeamQ). This comprised one theme that proved to be strong and reliable throughout the whole validation process. Clinical teachers seem to know very well what kind of leader they need and what they expect of a leader. This applies in particular for the item: I entrust the organization of teaching activities to the program director. The clinical teachers evidently entrust the teaching task to the program director. A frequently heard statement by clinical teachers and program directors was that the clinical teachers are particularly pleased for somebody to take the lead, and to take on the lion's share of the teaching tasks.

The third finding from the study on the leadership of program directors provided insight into four profiles of leadership: 'captains', 'carers', 'professionals' and 'team players'. It makes a difference whether the leader acts as a strict so-called 'captain', with a great feeling of responsibility, focusing on the rules, or whether he/she adopts the style of a relaxed 'team player' who attentively enjoys the talents of the clinical teachers and regards leadership as a game. Within all four different leadership profiles there seems to be a dominant preference for developing collectivity by providing information. However, simply providing information is not enough for investing in collective ambitions. The program directors have less experience with promoting team learning, while promoting learning is one of the main tasks and responsibilities of teachers in order to provide a positive learning environment and to safeguard the quality of postgraduate medical training. Team-based learning enables the members to practice and develop individual team skills, work better together, and improve overall team performance. ${ }^{17,18}$

Finally, the findings of the study on team interaction between clinical teachers highlighted the power struggle between the program director, the deputy director and the head of the medical department. This power struggle generated a tense atmosphere during formal meetings, with team members interrupting one another and talking among themselves, causing clinical teachers to drop out. Hardly any questions were asked, there was no drilling down on any questions that were asked, and talking about mistakes mainly centered on the question of blame. This study also showed that the program directors were aware of their leadership position as role models for 'speaking up' during the meetings. They indicated that they felt responsible for the teaching and for the proper running of the meetings. 
We learn from the literature that team leadership behaviors are important to achieve a team result. ${ }^{13,19,20}$ According to $\mathrm{McD}$ aniel, there are three features that characterize a strategic leader: (1) developing a collective mindset, (2) focusing on collaborative learning and (3) designing the teaching organization. ${ }^{21}$ This is how the strategic leader supports the team members in anticipating external changes. The program director, who has a formal leadership position within the teaching team, has to unite the individual expectations of team members and promote a common understanding regarding the expected professional performance of residents. ${ }^{22}$ In line with strategic leadership, Weggeman also emphasizes the importance of managing professionals by inspiring them and developing collective ambitions. ${ }^{23}$

We can conclude that the program directors focus on carrying out the teaching tasks and because less attention is paid to investing in collectivity, they support teamwork in clinical teaching teams only to a limited degree.

\section{The nature of teamwork and the supporting role of leadership}

In answering the research question on the nature of teamwork, we can make a number of different statements. Clinical teachers certainly do show individual results in the training of residents, although these results are hardly presented as a joint team result. Clinical teachers carry out independent teaching activities individually that are not well-embedded in a collective ambition or in a system of mutually influencing activities. This weakens the team result. The clinical teachers use teamwork qualities rather unilaterally and there is limited evidence of team learning within the teaching team. It is also because of the primarily action-oriented team process among clinical teachers and the relative lack of attention to any transition and interpersonal team process that there is a lack of collectivity in postgraduate medical training. Our studies suggest that the nature of the teamwork engaged in by clinical teaching teams can be summed up as follows: all clinical teachers do train residents, but not together as a team.

The answer to the second research question about the support for teamwork by the program director is closely related to the nature of teamwork as it has been described. 
The program directors are seen as a sort of 'super-teacher', who focus on the action process, a role that is regarded by the clinical teachers as extremely important. The program directors invest very little in the collective, professional ambition of the clinical teachers, and the support for teamwork to safeguard the quality of the postgraduate medical training appears to be limited. In this thesis there is little or no visible evidence of a program director being given or taking the opportunity to learn, together with the clinical teachers, how to be a teaching team.

\section{Strengths and Limitations}

We will discuss the strengths and limitations of the studies before formulating the practical and theoretical implications of this thesis. The structure of the studies, in which we first examined the experiences of the clinical teachers on the basis of a qualitative research design, is something we regard as one of the strengths of this thesis. The subsequent studies are all based on the results of earlier findings, which makes the research in this thesis consistent and strong. Secondly, a number of different, creative research designs have been applied, such as, for example, the observation and interview studies of teaching team interaction. A further strength is the sound application of the methodology throughout the whole thesis. All studies are based on thorough and relevant literature, and this was consistently and carefully compared with the data from practice, so that the discussion sections provide a valuable contribution to the development of the theory. Finally, the voice of the clinical teacher has been heard throughout all the studies. The strength of our approach is also that we did not adapt any of the existing models of the effectiveness of teamwork, but used an open-minded, appreciative and exploratory approach to arrive at an understanding and clarification of the views and experiences of clinical teachers. By promoting an open attitude and demonstrating a sincere interest in the experiences of the target group, it has been possible to gather a wealth of relevant data. We were never short of participants, which meant we always had very rich data available.

The limitations inherent in the various studies are discussed in the individual chapters, so we will only address here the general research limitations. The first limitation is 
that the studies were conducted in one specific setting: postgraduate medical training in the Netherlands. It would be useful to compare our findings to those of similar research in other countries with other cultures and beliefs. We could imagine that research on the teamwork of clinical teachers within different cultures with other methods of working together and different professional and hierarchical relations might generate a broader insight into the joint functioning of clinical teachers in postgraduate medical training.

Secondly, this thesis is based on perceptions and behaviors of clinical teachers and as such does not present the residents' views or experiences, while they are also to some extent members of the clinical team. It would be interesting to study the experience of residents with teamwork in clinical teaching teams. Finally, we see a limitation in the fact that, in order to answer the question posed in this thesis about the nature of clinical teaching teams and the supportive role of leadership, not all facets of teamwork and leadership have been addressed. It would be useful to carry out research on these other aspects of teamwork, such as team composition and size, over a longer period.

\section{Implications for future research}

This thesis lays a theoretical foundation for future research on the functioning of clinical teaching teams. First of all, a validated evaluation instrument (TeamQ) is now available, with which follow-up research can be carried out on different issues. TeamQ comprises 48 ideal typical items that together give an indication of the quality of teamwork within postgraduate medical training. An interesting follow-up research study would focus on what makes a clinical teaching team function excellently, or not; what the residents' perception of this is and what we can learn from it about the quality of postgraduate medical training. In addition, using the results of the TeamQ we can establish a relationship between the learning climate as experienced by the residents and the climate of teamwork among the clinical teachers. It is well known that an individual faculty's teaching performance evaluations are positively affected by better learning climates in residency programs. ${ }^{24,25}$ It would be valuable to study 
whether and how the quality of the teamwork among clinical teachers contributes to the quality of the learning climate.

A logical step in follow-up research would also be to focus attention on the dynamics between clinical teachers and the program director in developing team learning in a clinical teaching team. This would imply longitudinal studies that more thoroughly observe team learning of clinical teaching teams. ${ }^{7,26}$ Within a longitudinal study, using the TeamQ evaluation instrument, it would be possible to study the effect of interventions aimed at acquiring individual teamwork qualities, developing team learning and investing in collective ambitions with shared goals. Future research could also focus on developing personal leadership qualities in relation to the results of the TeamQ. Leadership qualities, such as investing in collective ambitions, inspiring team members and encouraging team learning can be developed by reflecting on the four leadership profiles of the program director. International comparative research on leadership, teamwork and 'speaking up' would also be useful. In a follow-up study we advocate focusing not on what participants think about teamwork, but specifically on what they actually do and demonstrate.

\section{Implications for medical education in practice}

If the clinical teachers operate as a team, they will be able to:

- communicate the collective professional ambitions more forcefully to residents, and to fellow professionals within the organisation and within the medical society;

- $\quad$ promote a climate of learning and giving feedback in a constructive way, to strive for excellent professional performance;

- anticipate 'as a change agent' the continuously changing context within postgraduate medical training; ${ }^{27}$

- carry out actions structurally to improve patient care by monitoring and improving the quality of postgraduate medical training.

But just how does a team become a team? What can we learn from the results of this thesis in developing teamwork in clinical teaching teams? 


\section{Reflection on teamwork qualities}

First and foremost, this thesis contributes to raising awareness among clinical teachers of the importance of teamwork in medical training. This is the first step in creating the urgency to practice and develop teamwork qualities (the belief in teamwork) and to allow clinical teachers to experience the positive effects of teamwork. Using the TeamQ tool, teachers can reflect in a structured way on their own teamwork qualities, which can be seen as an important first step in the transition towards more mutual engagement among clinical teachers. ${ }^{11}$ The insights into clinical teachers' selfreflections on teamwork may be helpful in creating opportunities for them to make the transition to a more effective clinical teaching team.

\section{Investing in collective ambition}

Discussing the results of the TeamQ tool with one another will make way for openness about the quality of teamwork in clinical teaching teams. Thereafter, motivated by collective ambitions, the next step can be made in teamwork. Striving for good performance ${ }^{28}$ can play a key role in formulating joint ambitions in postgraduate medical training and the quality of patient care. It is important here that the step from measuring performance to improving performance should be a joint activity, and that it should be understood that improvements are not one-off actions, but a continuous cycle of quality improvements, commonly known as the plan-do-study and action cycle. ${ }^{29}$ In order to practice accountability and to monitor - and where necessary improve- the quality of their teamwork, the clinical teaching teams should regularly evaluate their teamwork using the TeamQ as part of the quality management of postgraduate medical training.

\section{Investing in team learning}

These steps in team development can only be made if the clinical teaching team regards team learning as an element in effective teamwork. ${ }^{30-32}$ It is a matter of implementing and learning at the same time: actually getting work done while figuring out how to do it better. Team-based learning enables the members to develop individual teamwork qualities, work better together, and improve overall team results. ${ }^{17,18}$ Moreover, promoting learning is one of the main tasks and responsibilities of clinical teachers 
in order to provide a positive learning environment and to safeguard the quality of postgraduate medical training. Promoting team learning within the whole team, given the various roles that each team member fulfills, is an important focal area for the program director. Leadership skills can be learned; it is possible to learn how to resolve conflicts, to reinforce shared values and cooperative goals and to stimulate team learning among team members. ${ }^{33}$ The program directors build on the collective ambitions of the team to inspire team members, and empower the clinical teachers on the basis of their personal, unique talents.The program directors can reflect on their leadership qualities on the basis of our four profiles and develop leadership skills, at the same time as clinical teachers develop team learning.

\section{Anticipate external changes}

By creating a sense of collectivity, clinical teaching teams will be able to broaden the context, share the responsibility for teamwork with the residents and create multi-specialist teams. The investment in social ambitions extends further than the individual clinical teaching team. Weggeman refers to this situation as a phase of unification and solidarity that can come about in organizations once team members have reached a mutual stance on the ambition to produce a work of fairness, which they could not achieve individually. ${ }^{34}$ In this situation, teamwork and learning together within a clinical teaching team will be an example and an inspiration to the residents, for other disciplines within the hospital organization and the medical society.

\section{'Just do it' (Quote from a program director).}

The most important implication of this thesis is that team development is essential for continuous improvement of the quality of postgraduate medical training. This thesis can make a contribution to team development with the TeamQ evaluation instrument, the four leadership profiles for the program director and the findings of the team interaction during the formal teaching team meetings. The most important recommendation may well be that clinical teachers without doubt have the courage to take the first step towards one another and towards the continuous improvement of postgraduate medical training and achieving high-quality patient care. 


\section{Valorisation}

- The TeamQ instrument has been validated, is available in digital form and can be used as a tool to evaluate teamwork within clinical teaching teams. The instrument can be used within all of the more than 25 medical specialties within health care. The expectation is that by improving the teamwork between clinical teachers, the quality of postgraduate medical training and consequently the quality of patient care will be improved.

- The use of TeamQ is particularly recommended when key elements of teamwork are undergoing change, for example changing of key positions and resolving problems, including teamwork problems, or implementing major projects (such as preparing for a formal visitation). By gaining insight into the strong and weak points of their teamwork, clinical teams can anticipate these and work towards optimizing their performance within the team, so that the clinical teaching team is properly equipped for its task.

- The four profiles of leadership have been developed into a training program in which the program director can reflect on his or her leadership qualities. The results of the leadership study have been converted into 12 statements about such issues as leadership vision, the view of human nature and how the task in hand is interpreted. As part of the training program, the program directors formulate the ambitions for postgraduate medical training and set goals for investing in making these goals collective within the clinical teaching team.

- The method of data collection on speaking up in the team interaction during the teaching meetings is used in clinical teaching teams that want to improve their meeting culture. The current situation is discussed in advance with the program director, who in many cases is the chairperson of the teaching meeting and his or her deputy, in order to determine what the observation will focus on. Following the observation of the teaching team meeting, the audio fragments are discussed with the program director and his or her deputy in order to arrive at an analysis of the strengths and the points for improvement. This method initiates an awareness of the importance of efficient meetings and focusing not only on 'Let's keep things social and relaxed.' Team interaction using 'speaking up' 
is an excellent means to achieve open and transparent communication in order to make concrete agreements about safeguarding and improving the quality of medical postgraduate teaching. 


\section{References}

1. Burke CS, StagI KC, Salas E, Pierce L, Kendall D. (2006) Understanding team adaptation: a conceptual analysis and model. The Journal of applied psychology, 91(6), 1189-1207.

2. Salas E. (2007) Fostering Team Effectiveness in Organizations: Toward an Integrative Theoretical Framework. In: John William Shuart BS, Will Spaulding,Jeffrey Stephen Poland, ed. Modeling complex systems. Lincoln: University of Nebraska Press.

3. Salas E, Sims DE, Burke CS. (2005) Is there a "Big Five" in teamwork? Small Gr Res, 36(5), 555-599.

4. DeShon RP, Kozlowski SWJ, Schmidt AM, Milner KR, Wiechmann D. (2004) A multiplegoal, multilevel model of feedback effects on the regulation of individual and team performance. Journal of Applied Psychology, 89(6), 1035-1056.

5. Nadler DA. (1979) The effects of feedback on task group behavior: A review of the experimental research. Organ Behav Hum Perf, 23(3), 309-338.

6. Birden H, Glass N, Wilson I, Harrison M, Usherwood T, Nass D. (2014) Defining professionalism in medical education: a systematic review. Medical teacher, 36(1), 4761.

7. Mathieu J, Maynard MT, Rapp T, Gilson L. (2008) Team effectiveness 1997-2007: A review of recent advancements and a glimpse into the future. J Manage, 34(3), 410476.

8. Witman Y, Smid GAC, Meurs PL, Willems DL. (2011) Doctor in the lead: balancing between two worlds. Organization, 18(4), 477-495.

9. Lemieux-Charles L, McGuire WL. (2006) What do we know about health care team effectiveness? A review of the literature. Medical Care Research and Review, 63(3), 263300.

10. Lave J, Wenger E. (1998) Communities of practice.

11. Wenger E. (2000) Communities of practice and social learning systems. Organization, 7(2), 225-246.

12. Dornan T, Mann KV, Scherpbier AJ, Spencer JA. (2011) Medical education: theory and practice. Elsevier Health Sciences.

13. Burke CS, Stagl KC, Klein C, Goodwin GF, Salas E, Halpin SM. (2006) What type of leadership behaviors are functional in teams? A meta-analysis. Leadership Quart, 17(3), 288-307.

14. Stewart GL. (2010) The Past Twenty Years: Teams Research Is Alive and Well at the Journal of Management. J Manage, 36(4), 801-805.

15. Neily J, Mills PD, Young-Xu Y, et al. (2010) Association between implementation of a medical team training program and surgical mortality. JAMA, 304(15), 1693-1700.

16. Senior B, Swailes S. (2007) Inside Management Teams: Developing a Teamwork Survey Instrument. British Journal of Management, 18(2), 138-153.

17. Hammick M, Olckers L, Campion-Smith C. (2009) Learning in interprofessional teams: AMEE Guide no 38. Medical Teacher; 31, 1-12.

18. Magrane D, Khan O, Pigeon Y, Leadley J, Grigsby RK. (2010) Learning about teams by participating in teams. Academic medicine: Journal of the Association of American Medical Colleges, 85(8), 1303-1311.

19. Lieff SJ, Albert M. (2010) The mindsets of medical education leaders: how do they conceive of their work? Academic medicine : journal of the Association of American Medical Colleges, 85(1), 57-62.

20. Lingard L, Vanstone M, Durrant M, et al. (2012) Conflicting messages: examining the dynamics of leadership on interprofessional teams. Academic medicine : Journal of the Association of American Medical Colleges, 87(12), 1762-1767. 
21. McDaniel RRJ. (1997) Strategic Leadership: A View from Quantum and Chaos Theories. Health Care Management Review, 22(1):21-37.22(1):21-37.

22. Scheele F, Van Luijk S, Mulder H, et al. (2014) Is the modernisation of postgraduate medical training in the Netherlands successful? Views of the NVMO Special Interest Group on Postgraduate Medical Education. Medical teacher, 36(2), 116-120.

23. Weggeman M. (2007) Leidinggeven aan professionals? Niet doen! Over kenniswerkers, vakmanschap en innovatie. Schiedam, Scriptum.

24. Lombarts KM, Heineman MJ, Scherpbier AJ, Arah OA. (2014) Effect of the learning climate of residency programs on faculty's teaching performance as evaluated by residents. PloS one, 9(1), e86512.

25. Boor K, Van D, V, Teunissen P, Scherpbier A, Scheele F. (2011) Development and analysis of D-RECT, an instrument measuring residents? learning climate. Medical teacher, 33.10, 820-827.

26. Lingard L, McDougall A, Levstik M, Chandok N, Spafford MM, Schryer C. (2012) Representing complexity well: a story about teamwork, with implications for how we teach collaboration. Medical education, 46(9), 869-877.

27. Frenk J, Chen L, Bhutta ZA, et al. (2010) Health professionals for a new century: transforming education to strengthen health systems in an interdependent world. Lancet, 376(9756), 1923-1958.

28. Lombarts MJMH. (2014) Professional Performance van Artsen; tussen tijd en technologie. Rotterdam, 2010 Uitgevers.

29. Varkey P, Reller MK, Resar RK. (2007) Basics of quality improvement in health care. Mayo Clinic proceedings, 82(6), 735-739.

30. Edmondson AC. (2012) Teaming: How organizations learn, innovate, and compete in the knowledge economy. San Francisco, John Wiley \& Sons.

31. Edmondson AC, Lei Z. (2014) Psychological Safety: The History, Renaissance, and Future of an Interpersonal Construct. Annual Review of Organizational Psychology and Organizational Behavior; 1: 23-43.

32. Egan T, Jaye C. (2009) Communities of clinical practice: the social organization of clinical learning. Health, 13(1), 107-125.

33. Mitchell R, Parker V, Giles M, Boyle B. (2014) The ABC of health care team dynamics: Understanding complex affective, behavioral, and cognitive dynamics in interprofessional teams. Health care management review, 39.1, 1-9.

34. Weggeman M. (2003) Provocatief adviseren: Organisaties mooier maken. Scheidam, Scriptum. 



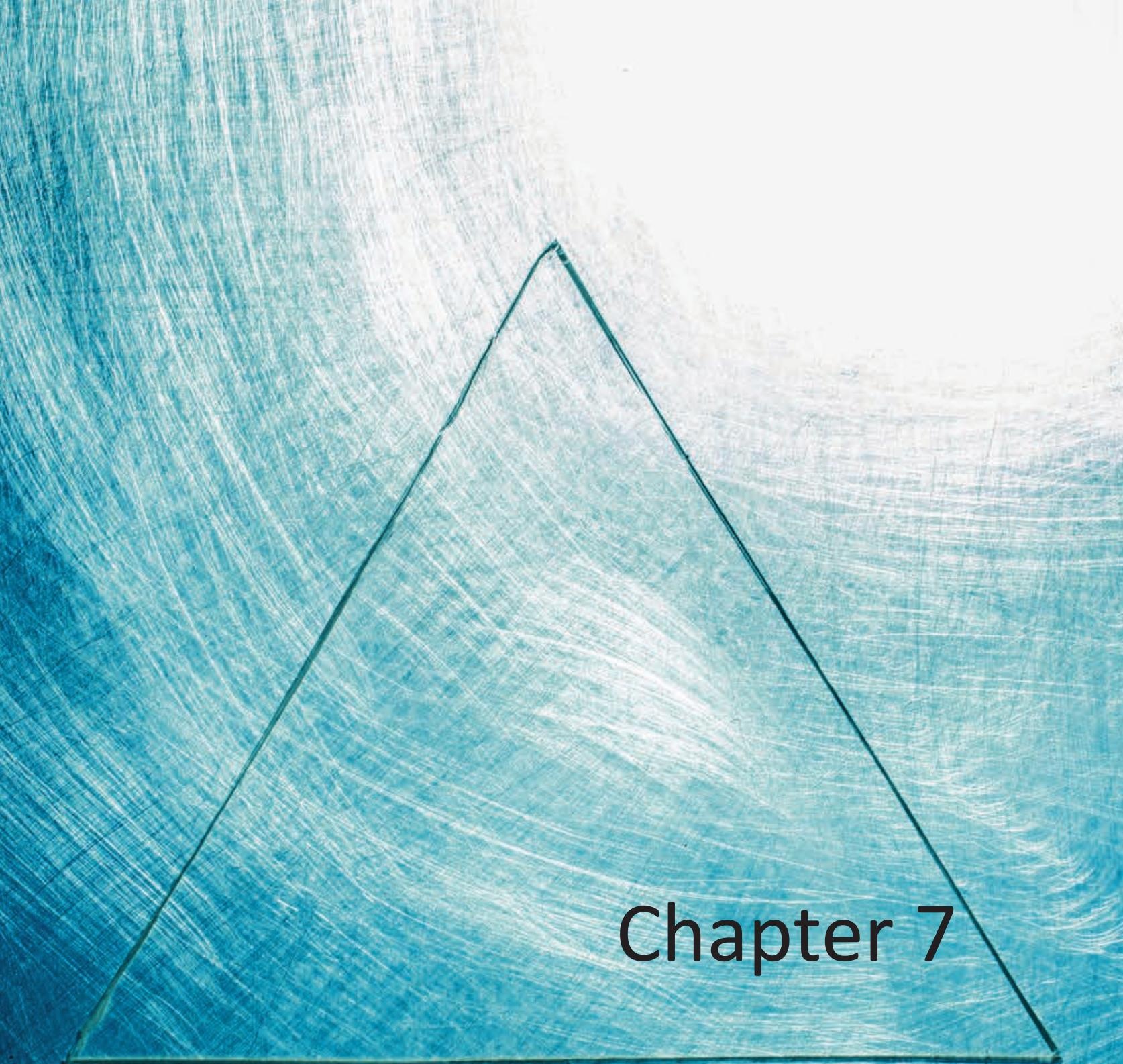

Mow

Summary

Summary in Dutch

Dankwoord

Curriculum Vitae 
126 | Chapter 7 


\section{Summary}

Chapter 1 forms the introductory chapter of this thesis. We describe the key constructs of this thesis, the problems, the aims and the overarching research questions. The thesis explores teamwork among clinical teachers in postgraduate medical training. Doctors today will have a competency-based training and will hopefully be better equipped to anticipate the changes within professional medical practice. There are two key terms for change in healthcare: patient-centered and team-based. Jointly carrying out an objective and transparent workplace-based assessment, based on the CanMeds competences, represents a significant challenge for clinical teachers. Since 2011, the joint responsibility of clinical teachers for the quality and practice of postgraduate medical training has been set out explicitly in Dutch legislation. In this legislation, clinical teachers are structured as a team and are referred to as 'members of a clinical teaching team'. Postgraduate medical training is organized in different ways in different parts of the world. Nonetheless, there is a single universal principle, namely that clinical teachers, also referred to as clinician educators, are jointly responsible for the quality of the discipline-specific training of residents in the workplace. What teamwork among clinical teachers consists of, why it is important and what it can contribute to the quality of postgraduate medical training is key to this thesis. The aim is to deconstruct the teamwork of clinical teachers, on the basis of two overarching research questions: What is the nature of teamwork among clinical teachers? and What is the role of leadership in supporting the teamwork of clinical teachers? The intention is to use this thesis to further deconstruct teamwork in order to contribute to the development of knowledge on teamwork and leadership. It is also intended as a means of offering guidelines to clinical teachers to improve disciplinespecific teamwork in the training of residents. Chapter 1 shows that four studies have been undertaken that aim to clarify teamwork among clinical teachers and to examine the role of the program director. In answering the overarching research questions we were inspired by three theoretical perspectives. First, we knew from medical professionalism that clinical teachers favour a collegial and loyal attitude towards colleagues who hold a fundamentally different opinion about the residents. Second, from the perspective of social cultural learning theory we learned that clinical teachers, 
often self-trained according the traditional teacher/apprentice training paradigm, train residents who will learn in the workplace and participate in the clinical teaching team. Finally, we knew from the organizational perspective that clinical teachers, used to training residents in a one-to-one setting, are now teaching under the leadership of the program director together with the other clinical teachers, who are all jointly responsible for the quality of the postgraduate medical training. The main issues can therefore be said to be teamwork and leadership.

In chapter 2 we report the first step in research on the nature of teamwork and the supporting role of leadership in teamwork. In a qualitative study we explored clinical teachers' experience with teamwork, with the research question: How do clinical teachers work together as a team in delivering postgraduate medical training? Data were collected during six focus group interviews ( $n=50$ clinical teachers) and analysed using the thematic network system. There were 7 related themes to the phenomenon of teamwork as experienced by the clinical teachers:

- the clinical teachers were more passionate about clinical expertise than about specific knowledge about teaching and teamwork;

- $\quad$ residents are trained by many teachers. They vainly look for information and understanding about clinical teachers' professional standards and what they expect from residents;

- $\quad$ neither the program director nor the other members of the teaching team had a clear idea of the director's role with regard to the development of teamwork;

- discussions among clinical teachers were mostly concerned with residents' performance and the division of teaching tasks;

- the clinical teachers had a clear understanding of the structural elements of residency training, including formal meetings and decision-making;

- it was not common practice for clinical teachers to use feedback in the teaching team;

- the clinical teachers felt under external pressure to be accountable for team performance with regard to residency training.

The results give ample illustration of the lack of effective teamwork in delivering postgraduate medical training. The lack of clarity with regard to the program director's 
role as team leader contributed to the lack of coherence in teams of clinical teachers. Social cultural learning theory describes the importance of engagement with other clinical teachers and the creation of meaningful connections. From the organizational literature we learned the importance of the program director in developing a shared vision, developing individual teamwork qualities and steering on mutual team results.

In chapter 3 we present a mixed method study to evaluate teamwork among clinical teachers based on the seven themes of teamwork experienced by the clinical teacher. Even though many measurement instruments are available for evaluating teamwork in health care teams, no particular instrument is specific enough for use in teaching teams in postgraduate medical training. This study aimed to develop and validate such an instrument (TeamQ) and to explore how clinical teachers appraise their current levels of teamwork. We used a mixed-methods approach based on a modified Delphi consensus procedure, followed by psychometric analyses of the instrument. We analyzed the content validity, based on the results of chapter 2. An expert group of 13 program directors, 7 clinical teachers, 6 residents and 5 educationalists assessed the items on the basis of the Delphi consensus method. A web-based TeamQ instrument was developed to test the validity and reliability of the remaining items (51). In total, 929 clinical teachers from 114 teaching teams of multiple specialties and multiple organizations completed the evaluation. The median team response was 7 evaluations per team. The principal component analysis included 8 factors, labeled as 'task expertise', 'team expertise', 'decision-making', team leadership', 'feedback culture', 'team results', 'engaging residents' and 'residents' empowerment'. The reliability coefficients of the TeamQ scales ranged from 0.75 to 0.93 . The generalizability analysis revealed that 5 to 7 evaluations were needed to obtain internal reliability coefficients of 0.70. In terms of teamwork, the clinical teachers scored 'residents' empowerment' as the highest TeamQ scale and 'feedback culture' as the area that would most benefit from improvement. This study provides initial evidence of the validity of an instrument for measuring teamwork in teaching teams. The high response rates and the low number of evaluations needed for reliably measuring teamwork indicate that TeamQ is feasible for use in the evaluation of teamwork in teaching teams. The use of TeamQ may be the first step in an improvement process. We know from the literature 
the importance not only of measuring the quality of teamwork, but also of reflecting on the TeamQ results and then, together as a clinical teaching team, formulating an action plan to achieve the real improvement.

In chapter 4 we focus on the leadership of the program director. In providing high quality postgraduate medical training, program directors have a formal leadership position within a teaching team. It is not clear how they fulfill this leadership role in actual practice; consequently, too little is known about the activities that are needed to develop leadership qualities aimed at improving teamwork among clinical teachers. The results of chapter 2 show that neither the program director nor the other members of the clinical teaching team had a clear idea of the director's role with regard to the development of teamwork. In this qualitative study we aim to explore the role of the program director as a strategic leader, based on the research question: What are the experiences of program directors with strategic leadership? Strategic leadership in health care appears to best reflect the role of the program director because a strategic leader prepares the team to anticipate external changes: 1) developing a collective mindset, 2) focusing on collaborative learning, and 3) designing the teaching organization. Promoting learning is one of the main tasks and responsibilities of clinical teachers in order to provide a positive learning environment and to safeguard the quality of advanced specialty training. We conducted an interview study using the principles of phenomenography to investigate the experience of the program directors. This approach focuses on the way a group experiences the phenomenon under investigation. Fourteen program directors from different hospitals and different specialties participated in this study. Data were iteratively analyzed using the template analysis method, based on the three features of strategic leadership; 'task interpretations' were added to the initial template. Based on the experiences of the program directors, we identified four leadership profiles: 'captains', 'carers', 'professionals' and 'team players'. The 'team players' come closest to integrally applying the strategic leadership features. For all four profiles there seems to be a preference for developing collectivity by means of providing information. Program directors have less experience with promoting collaborative learning and the teaching organization is designed to be task-oriented. If the program directors could shift their 
focus to developing team learning and become more learning-oriented leaders, the clinical teaching team would be better able to anticipate external developments. This could be expected to improve the quality of postgraduate medical training.

In chapter 5 we explored the team interaction among clinical teachers during a formal teaching meeting. In an exploratory study based on ethnographic principles we analyzed how clinical teachers demonstrate 'speaking up' in formal teaching team meetings and what factors influence this. 'Speaking up' is defined as a sincere and direct manner of communication between individuals and includes such activities as asking questions, seeking feedback and discussing mistakes. 'Speaking up' has been shown to have a preventive effect on human error. Little is known about 'speaking up' in disciplinespecific teamwork among clinical teachers for the quality of postgraduate medical training. The study findings may be useful when developing interventions to promote 'speaking up' among clinical teachers. Ten teaching teams from different hospitals and different specialties participated in the study. The teaching team meetings were observed, audio recorded and analyzed. Subsequently, in an interview setting, the program directors reflected on 'speaking up' behaviors during the meetings, based on specific audio fragments. The program directors are responsible for monitoring and promoting effective teamwork, which includes encouraging 'speaking up' among team members. All audio fragments were analyzed interactively using a template analysis based on Edmondson's six behaviors of 'speaking up': 1) asking questions, 2) sharing information, 3) seeking help, 4) experimenting with unproven actions, 5) talking about one's own mistakes, and 6) seeking feedback.

During the teaching team meetings, most of Edmondson's six behaviors of 'speaking up' were exhibited, but one, 'experimenting with unproven actions', was observed only very rarely. Both program directors and clinical teachers displayed the behavior of providing information, which is related to the behavior of 'sharing information'. 'Talking about mistakes' occurred in a general sense and without commitment to improvement activities. 'Questions' were asked, but at times without waiting for the answer and they were often phrased as closed questions. 'Seeking help' was mainly displayed by clinical teachers when seeking assistance from colleagues in implementing the teaching task. 'Seeking feedback' was expressed by asking for feedback about established policy 
or about personal performance. Three type of factors were identified that appear to influence speaking up by clinical teachers: relational factors (power, leadership, feeling safe and handling conflict), cultural factors (history, meeting and feedback), and professional factors (commitment to teaching, contribution by residents and discipline-specific). Clinical teachers demonstrate behaviors of speaking up during teaching team meetings; however problematic topics only appear to be discussed to a limited degree with no action being taken. Mistakes and conflicts are mainly discussed in a general sense, and are often neither directed at the individual nor adequately result-oriented. This means that the potential positive effect that 'speaking up' could have on the quality of postgraduate medical training is largely lacking. If clinical teachers are to develop 'speaking up' behaviors, it is important to take into account the relational, cultural and professional factors that influence 'speaking up' in order to stimulate sharing information during the teaching team meetings.

Chapter 6 is the chapter of reflection and discussion, in which we answer the two main research questions and discuss the strengths and limitations of this thesis. We can conclude that the nature of the teamwork engaged in by clinical teaching teams can be summed up as follows: all clinical teachers do train residents, but not together as a team. The program director invests little in the collective, professional ambition of the clinical teachers, and the support for jointly taking responsibility for the quality of the postgraduate medical training appears to be limited. It is well known from the literature that practicing and developing individual teamwork qualities by members of a team, along with working towards shared goals, are widely regarded as the core of teamwork. Teamwork should in fact be seen as a verb, whereby feedback and evaluations are important factors in working and learning together.

In chapter 6, the final chapter of this thesis, we give the answers to the research questions in the form of the four most important findings.

1. Clinical teachers pay little or no attention to practicing and developing individual teamwork qualities. This applies both in the area of the understanding of teamwork and the skills of giving and receiving feedback, managing conflict, and decision-making, as well as the belief in teamwork in postgraduate medical training. 
2. The team process is primarily an action process, whereby the transition process and the interpersonal process are scarcely applied at all by clinical teachers. The action process is above all focused on implementing teaching tasks, but results in hardly any concrete agreements being made about the training of residents, the monitoring of the progress of team results and giving one another feedback. If any interpersonal processes do occur, these seem above all aimed at avoiding conflicts and preserving harmonious relations. The transition process aimed at the mission statement and strategic goals of the teaching team is scarcely noticeable in the studies.

3. The team results as such reflect the type of teamwork that clinical teachers want to strive for, and are in terms of the nature of teamwork, insufficiently demonstrated in this thesis.

4. The primary focus of the program director is on carrying out the teaching tasks, and as a consequence, less attention is paid to investing in collectivity and team learning. Clinical teachers indicate that they want a leader who acts as a kind of 'super-clinical teacher' who takes on the lion's share of the teaching tasks.

A particular strength of this thesis is the structure of the studies. The subsequent studies are all based on the results of earlier findings, which makes the research in this thesis strong and consistent. Further strengths are the number of different creative research designs that have been applied in this thesis. The main limitation of the work is that the studies were conducted in one specific setting: postgraduate medical training in the Netherlands. A further limitation is that this thesis is based on perceptions and behaviors of clinical teachers and as such does not discuss the residents, while they are also to a greater or lesser extent members of a clinical team. It would be useful to study the experience of residents with teamwork in clinical teaching teams. This thesis lays a theoretical foundation for more future research on the functioning of clinical teaching teams. The results of the validated evaluation instrument (TeamQ) are available for follow-up research on the relationship between the quality of teamwork amongst clinical teachers and the quality of the learning climate. A logical step in follow-up research would also be to pay attention to the dynamics between clinical teachers and the program director in developing team learning in a clinical teaching team. This would imply longitudinal studies that more 
thoroughly observe team learning of clinical teaching teams. Within such a study, using the TeamQ evaluation instrument, it would be possible to study the effect of interventions aimed at developing individual teamwork qualities, improving team learning and investing in collective ambitions with shared goals.

The practical implication of this thesis is its contribution to raising awareness among clinical teachers of the importance of teamwork in postgraduate medical training. This is the first step in creating the urgency to apply and develop teamwork qualities (the belief in teamwork) and to allow clinical teachers to experience the positive effects of teamwork. In order to practice accountability and to monitor, and where necessary improve, the quality of their teamwork, the clinical teaching teams should regularly evaluate their teamwork using the TeamQ as part of the quality assurance of postgraduate medical training. The most important implication of this thesis is that team development is essential for continuous improvement in the quality of postgraduate medical training. The steps in team development can be made if and when the clinical teaching team really takes team learning as an element of effective teamwork seriously. It is a matter of implementing and learning at the same time: getting work done while figuring out how to do it better. The program directors can reflect on their personal leadership qualities on the basis of our four profiles and can develop their leadership skills, at the same time as clinical teachers develop team learning. By creating a sense of collectivity, clinical teaching teams will be able to broaden the context, share the responsibility for teamwork with the residents and create multi-specialist teams. The recommendation may well be that clinical teachers together make the step towards the continuous improvement of postgraduate medical training and achieving high-quality patient care. 


\section{Samenvatting}

Hoofdstuk 1 kan gezien worden als een introducerend hoofdstuk waarin we de kernbegrippen van dit proefschrift, de probleemstelling, het doel en de hoofdvragen beschrijven. Het is de samenwerking tussen klinische opleiders in het kader van de medisch specialistische vervolgopleiding, die in dit proefschrift wordt verkend. Internationale ontwikkelingen van de medische specialistische vervolgopleiding anticiperen op devoortdurendeveranderingen binnen de medische beroepsuitoefening en zijn noodzakelijk om de dokter van de toekomst op te leiden. Kernwoorden van de verandering zijn patiëntgerichtheid en samenwerken. Een belangrijke uitdaging hierin is voor de klinische opleiders om het competentiegericht curriculum gebaseerd op de CanMeds, vorm te geven met nadrukkelijke aandacht voor het leren op de werkplek. Concreet betekent dit bijvoorbeeld: het gezamenlijk uitvoeren van een uniforme begeleidingsaanpak voor individuele arts-assistenten en het zorg dragen voor een objectief en transparant beoordelingssysteem. Om het samenwerken tussen opleiders te stimuleren en te waarborgen, is in Nederland in 2011, in de wet- en regelgeving de gezamenlijke verantwoordelijkheid van de opleiders voor de kwaliteit en uitvoering van de medische specialistische vervolgopleiding vastgelegd. Bekend is dat de medische specialistische vervolgopleidingen wereldwijd verschillend georganiseerd zijn, terwijl universeel is dat opleiders samen verantwoordelijk zijn voor de kwaliteit van het discipline-specifiek opleiden van arts-assistenten op de werkplek.

Het belang van samenwerken kennen we uit de literatuur, maar we weten onvoldoende hoe opleiders samenwerken binnen de medische specialistische vervolgopleiding. In dit proefschrift analyseren we de samenwerking van opleiders aan de hand van twee hoofdvragen: wat is de aard van de samenwerking van opleiders en wat is de rol van leiderschap in het stimuleren van de samenwerking tussen opleiders? Het is de bedoeling om hiermee bij te dragen aan de ontwikkeling van kennis over samenwerken en leiderschap. Daarnaast is het doel een handreiking te bieden aan opleiders om de samenwerking ten behoeve van het opleiden van arts-assistenten binnen een specialisme te verbeteren. Hoofdstuk 1 laat zien dat er vier studies in samenhang zijn uitgevoerd om inzichtelijk te maken hoe de samenwerking tussen opleiders verloopt en wat de rol van de formele opleider is. Om antwoord te geven op de 
hoofdvragen van dit proefschrift, dragen 3 verschillende theoretische perspectieven bij aan het verklaren van de bevindingen. Allereerst weten we vanuit het medische professionalisme dat opleiders, loyaal en liefst zonder het geven van kritiek, zich verhouden tot collega's die andere opvattingen hebben over het opleiden van de artsassistenten. Ten tweede laat het perspectief van sociaal-culturele leertheorie zien dat de opleiders, veelal zelf opgeleid vanuit een traditioneel leermeester - gezel relatie, arts-assistenten opleiden die willen participeren op de werkplek en erbij willen horen. Tot slot zien we vanuit het perspectief van de organisatiekunde dat de opleider, die gewend is zelfstandig arts-assistenten op te leiden, nu onder leiding van de formele opleider, gezamenlijk verantwoordelijkheid moet dragen voor het eindresultaat: de kwaliteit van de medische specialistische vervolgopleiding. Het komt dus aan op samenwerken en op leiderschap.

In hoofdstuk 2 rapporteren wij over de eerste stap in het onderzoek naar de aard van samenwerken en leiderschap, door de opleiders zelf aan het woord te laten over hun ervaringen. De onderzoeksvraag was: 'Hoe werken de opleiders samen als team in het opleiden van arts-assistenten?' In een fenomenologische studie werd data verzameld met behulp van 6 focusgroep discussies ( $n=50$ opleiders) en geanalyseerd met een 'thematische netwerktechniek'. Er bleken 7 met elkaar samenhangende thema's bepalend te zijn voor de samenwerking:

- opleiders zijn meer gepassioneerd over hun medische expertise dan over specifieke kennis over opleiden en samenwerken;

- arts-assistent heeft een meester-gezel relatie met veel meesters. Uniforme informatie over professionele standaarden en over het individuele niveau van functioneren, wordt gemist;

- zowel de formele opleider als de opleiders hebben geen helder beeld van leiderschap ten behoeve van het stimuleren van de samenwerking;

- discussiepunten tussen de opleiders zijn het functioneren van de arts-assistenten en het verdelen van opleiderstaken;

- de structuur van het opleiden ervaren opleiders als hiërarchisch, vanzelfsprekend en nuttig;

- $\quad$ voor het opleiden is feedback geven en ontvangen belangrijk en in de praktijk lastig uitvoerbaar; 
- de opleiders voelen een externe druk om als team verantwoording af te leggen over de kwaliteit van de medische vervolgopleiding.

We zien bij opleiders weinig aandacht voor (de ontwikkeling van) samenwerken ten behoeve van de medisch specialistische vervolgopleiding. De focus van de formele opleider ligt vooral op het uitoefenen van opleiderstaken en in mindere mate op het ontwikkelen van de samenwerking binnen het opleidingsteam. Daarnaast ervaren opleiders door externe ontwikkelingen wel een toenemende urgentie om de samenwerking te verbeteren. De sociaal-culturele leertheorie benadrukt het belang van onderlinge betrokkenheid en het verhelderen van sociale relaties tussen de opleiders. Uit de organisatiekunde leren we het belang van de leidinggevende rol van de formele opleider in het ontwikkelen van visie, het stimuleren van samenwerkingskwaliteiten en het sturen op gezamenlijke resultaten.

In hoofdstuk 3 borduren we voort op het tweede hoofdstuk en de 7 thema's die bepalend zijn voor de samenwerking. Om de effectiviteit van de samenwerking te beïnvloeden, wordt een meetinstrument ontwikkeld om de samenwerking in opleidingsteams te evalueren (TeamQ). Opleiders krijgen inzicht in de kwaliteit van de samenwerking, om deze waar nodig aan te passen aan de vernieuwde eisen van de medisch vervolgopleiding. Er zijn veel instrumenten beschikbaar die het functioneren van groepen meten, maar geen daarvan is specifiek ontwikkeld en gevalideerd voor opleidingsteams. Het doel van deze studie was om de inhoudelijke validiteit en de betrouwbaarheid van het evaluatie-instrument TeamQ te beoordelen. Daarnaast werd onderzocht hoe de opleiders de huidige situatie van samenwerken beoordelen. Voor het inhoudelijk valideren zijn de 7 thema's en de citaten van de opleiders uit de eerste studie vertaald in stellingen. Met behulp van een gemodificeerde consensus methode hebben een groep van 32 experts, bestaand uit 13 formele opleiders, 7 klinische opleiders, 6 arts-assistenten en 5 onderwijskundigen in 2 rondes de stellingen over samenwerken in opleidingsteams beoordeeld. Uiteindelijk bleven er 51 bruikbare stellingen over, waarmee een digitale versie van het instrument is ontwikkeld. Er participeerden 114 opleidingsteams, met 929 opleiders uit verschillende opleidingsziekenhuizen, van verschillende specialismen in dit onderzoek. Door middel van een factor analyse konden er 8 met elkaar samenhangende factoren en 48 ideaal 
geformuleerde criteria worden geïdentificeerd: 'opleidingstaken', 'samenwerken', 'besluiten nemen', 'leiderschap', 'feedback', 'resultaten', 'individueel opleiden', 'actief leren'. De betrouwbaarheid van de TeamQ schalen ligt tussen de 0.66 ('actief leren') en de 0.93 ('leiderschap'). De generaliseerbaarheid resulteerde in het feit dat er 5 tot 7 ingevulde evaluaties nodig zijn om een betrouwbaar interne consistentie ( 0.70$)$ te halen.

In termen van de huidige samenwerking waardeerden de opleiders het thema 'actief leren' als thema dat zij het meest toepassen. De laagste waardering kreeg het thema 'feedback', wat aangeeft dat hier meer aandacht aan besteed kan worden. Het hoge respons percentage en het geringe aantal evaluaties dat nodig is om betrouwbare meting uit te voeren, geeft vertrouwen in het gebruik van het TeamQ instrument in de praktijk. Het gebruik van de TeamQ kan gezien worden als eerste stap in kwaliteitscyclus van meten naar verbeteren. Vanuit de literatuur weten we dat het daarna belangrijk is om gezamenlijk op de evaluatiegegevens te reflecteren, deze te bespreken en te komen tot een verbeterplan.

In hoofdstuk 4 staat het onderwerp leiderschap van de formele opleiders centraal. De formele opleider is samen met de leden van het opleidingsteam, verantwoordelijk voor de kwaliteit van de medisch specialistische vervolgopleiding. De resultaten van hoofdstuk 2 toonden echter aan dat er geen helder beeld bestond over de rol van de formele opleiders. Uit de literatuur weten we hoe belangrijk een leider is voor het samenwerken in teams bij het uitvoeren van taken. In deze studie onderzochten we de ervaringen van de formele opleiders als strategisch leiders, om daarmee inzicht te geven in de wijze waarop formele opleiders leiding geven aan opleidingsteams. Strategisch leiderschap werd als uitgangspunt gekozen vanwege het aspect van samenwerkend leren. Concreet vanwege het voorbeeldgedrag dat opleiders laten zien als zij gezamenlijk leren van fouten en bereid zijn verbeterplannen te ontwikkelen, uitvoeren en evalueren. Dat gedrag kan stimulerend werken op het leren en ontwikkelen van de (samenwerkingskwaliteiten van de) arts-assistenten. Binnen het gedachtegoed van strategisch leiderschap speelt de leider een onmisbare rol in de uitvoering van drie met elkaar samenhangende taken: 1) het ontwikkelen van collectiviteit 2) de aandacht voor samenwerkend leren en 3) het organiseren 
van onderwijstaken. De onderzoeksvraag van de interview studie was: 'Wat zijn de ervaringen van de formele opleiders met strategisch leiderschap?' De studie werd uitgevoerd volgens de principes van de fenomenografie, waarbij de manier waarop een groep een fenomeen ervaart centraal staat en niet de opvattingen van individuen. Veertien formele opleiders namen deel aan deze interviewstudie: 8 vanuit de Universitair Medische Centra en 6 vanuit opleidingsziekenhuizen; 6 niet-chirurgisch, 4 chirurgisch en 4 vanuit ondersteunende specialismen. De gesprekken werden geanalyseerd met behulp van template analysis. Het initiële 'template' bestond uit de drie taken van de strategisch leider waar later de 'taakopvatting' aan werd toegevoegd. Uit hun ervaringen konden vier leiderschapprofielen worden geïdentificeerd: 'kapiteins', 'beschermers', 'professionals' en 'teamspelers'. De 'teamspelers' komen het dichtst bij het integraal vormgeven van strategisch leiderschap. Formele opleiders hebben minder ervaring met het stimuleren van het samenwerkend leren, terwijl de organisatie vooral gericht is op verdelen van opleiderstaken. Met behulp van de vier leiderschapsprofielen kunnen formele opleiders reflecteren op hun eigen leiderschap en experimenteren met het integraal toepassen van strategisch leiderschap. Daarmee is het opleidingsteam beter in staat om als een team op externe ontwikkelingen te anticiperen, wat mogelijk een positief effect zal hebben op de kwaliteit van de medische specialistische vervolgopleiding.

In hoofdstuk 5 beschrijven we de studie naar de teaminteractie tussen opleiders tijdens een opleidingsvergadering. In een kwalitatieve studie, gebaseerd op etnografische principes is onderzocht op welke wijze opleiders 'speaking up' demonstreren tijdens een formele opleidingsvergadering en welke factoren deze 'speaking up' beïnvloeden. 'Speaking up'- elkaar aanspreken- is gedefinieerd als een oprechte en directe interactie tussen individuen. Steeds meer onderzoek toont aan hoe belangrijk 'speaking up' is voor de kwaliteit van de patiëntenzorg. Onbekend is welke rol 'speaking up' speelt bij opleiders in de teaminteractie ten behoeve van de medische vervolgopleidingen. Er zijn 10 opleidingsteams geselecteerd aan de hand van een doelgerichte steekproef: 3 teams uit de Universitair Medische Centra en 7 uit de opleidingsziekenhuizen; 4 niet-chirurgische, 4 chirurgische en 2 vanuit ondersteunende disciplines. De opleidingsvergaderingen werden geobserveerd, er werden geluidsopnames van 
gemaakt en deze werden als zodanig geanalyseerd. Vervolgens werd een interview gehouden met de formele opleider aan de hand van geluidsfragmenten, waarin de formele voorzitter, als eindverantwoordelijke van het opleidingsteam, reflecteerde op de toepassing van 'speaking up' door de leden van het opleidingsteam. Alle audiofragmenten zijn iteratief geanalyseerd, met behulp van een ordeningsprincipe gebaseerd op de 6 gedragingen van 'speaking up': hulp vragen, feedback zoeken, informatie delen, vragen stellen, experimenteren en praten over fouten. Tijdens de opleidingsvergadering lieten opleiders vijf van de zes gedragingen van 'speaking up' zien. De opleiders verschaffen vooral informatie in plaats van dat zij 'informatie delen'. 'Praten over fouten' komt vooral in algemene zin voor, terwijl afspraken maken over verbetersuggesties om fouten te voorkomen, uitblijven. Het betreft echter vaak gesloten vragen, die regelmatig gesteld worden zonder antwoord af te wachten. 'Hulp zoeken' gebeurde net als 'feedback zoeken' vooral door de formele opleider bij de uitvoering van opleidingstaken. Factoren die van invloed blijken te zijn op de praktijk van 'speaking up' zijn relationeel (macht, veiligheid, conflicthantering), cultureel (historie, feedback - en vergadercultuur) en professioneel (rol van de arts-assistenten, betrokkenheid bij opleiden, karaktertrekken) van aard. Als er meer aandacht komt voor het inzetten en ontwikkelen van 'speaking up' door opleiders, zal dat mogelijk een positief effect hebben op het openlijk bespreken van problemen en fouten, waardoor concrete verbetersuggesties uitgevoerd kunnen worden. Kennis over de beïnvloedende factoren voor 'speaking up' leveren inzicht op in de manier waarop de samenwerking tussen opleiders bevorderd kan worden.

Hoofdstuk 6 is het hoofdstuk waarin we de vier onderzoeken te samen bediscussiëren, antwoord geven op de twee hoofdvragen en de sterke punten en de beperking van dit proefschrift behandelen. Het antwoord op de hoofdvragen naar de aard van de samenwerking van opleiders is samen te vatten als: opleiders leiden samen op, maar niet als team. De formele opleider investeert vooral in opleidingstaken en nauwelijks in de collectieve, vakinhoudelijk ambitie van opleiders, waardoor de stimulans voor samenwerken gering blijft. Bekend uit de literatuur is dat de inzet en ontwikkeling van individuele samenwerkingskwaliteiten door leden van een team, naast het werken met een gezamenlijk doel, beschouwd kan worden als het hart van samenwerken. 
Samenwerken is een werkwoord, waarbij feedback en evaluaties belangrijk zijn, zodat het samen werken én samen leren hand in hand gaat. In hoofdstuk 6, tevens het laatste hoofdstuk van het proefschrift, wordt het antwoord op de hoofdvragen toegelicht aan de hand van de vier belangrijkste bevindingen.

1) De klinische opleider is vooral een medische professional die aandacht heeft voor het ontwikkelen van medisch inhoudelijk expertise. De inzet en ontwikkeling van persoonlijke samenwerkingskwaliteiten ten behoeve van het opleiden van arts-assistenten zijn in de studies nauwelijks zichtbaar geworden. Concreet gaat het om kwaliteiten als: kennis over samenwerken, vaardigheden om feedback te geven en ontvangen, conflicten te hanteren en besluiten te nemen. En het gaat om een uitstraling, waaruit het belang van samenwerking voor de medische specialistische vervolgopleiding blijkt.

2) Het teamproces tussen de opleiders is vooral actiegericht, terwijl de transitieprocessen en de inter-persoonlijke processen door de opleiders weinig worden toegepast. Actiegerichte processen gaan over de uitvoering van opleidingstaken, alleen leiden deze processen nauwelijks tot concrete afspraken over het opleiden van de arts-assistent en het monitoren van de voortgang van gezamenlijke resultaten op het gebied van de kwaliteit van de medisch specialistische vervolgopleiding. Interpersoonlijke teamprocessen komen wel voor, maar die lijken vooral conflicten te vermijden en uit te zijn op het koesteren van de harmonie. De transitieprocessen die gericht zijn op ontwikkelen van missie, visie en strategische doelstellingen, zijn in de studies nauwelijks zichtbaar geworden.

3) De gezamenlijke teamresultaten worden door opleiders wel als belangrijk en nastrevenswaardig geacht, maar aantoonbare teamresultaten van een opleidingsteam waren in dit proefschrift nauwelijks merkbaar.

4) De formele opleider zet leiderschap vooral in voor het uitvoeren van opleiderstaken en is, omdat er minder aandacht is voor het investeren in collectiviteit en samenwerkend leren, in geringe mate stimulerend voor de samenwerking tussen opleiders in opleidingsteams. Opleiders lijken vooral een leider nodig te hebben als een soort 'super-opleider' die het leeuwendeel van de opleidingstaken op zich neemt. 
Sterk aan dit proefschrift is de opbouw van de studies, waarbij de vervolgonderzoeken gebaseerd zijn op eerdere bevindingen. Er zijn creatieve onderzoeksontwerpen toegepast en methodologisch consequent uitgevoerd. De beperkingen liggen vooral op het terrein van de specifieke Nederlandse setting, terwijl onderzoek naar de disciplinespecifieke samenwerking van opleiders uit verschillende culturen heel nuttig zou zijn. In de studies bleef ook het perspectief van de arts-assistenten onderbelicht. De implicatie van het proefschrift is dat er een theoretische basis is gelegd voor toekomstig onderzoek naar het functioneren van opleidingsteams. Met de resultaten van het gevalideerde evaluatie-instrument TeamQ kan er relatie gelegd worden tussen de kwaliteit van de samenwerking van opleiders en de kwaliteit van het leerklimaat. Binnen het longitudinaal onderzoek kan met behulp van het evaluatieinstrument TeamQ het effect van interventies gericht op het ontwikkelen van individuele samenwerkingskwaliteiten, het ontwikkelen van samenwerkend leren en de investering in collectieve ambities onderzocht worden. Voor de praktijk van de medisch specialistische vervolgopleiding is er met dit proefschrift een eerste stap gezet in het creëren van urgentie om samenwerkingskwaliteiten (en vooral het 'geloof in' samenwerken) in te zetten en te ontwikkelen om een positief effect van samenwerken van opleiders te laten ervaren. Met behulp van de TeamQ kunnen opleiders gestructureerd reflecteren op hun eigen samenwerkingskwaliteiten en dat kan gezien worden als een belangrijke stap in de transitie naar meer onderlinge betrokkenheid tussen opleiders. Door de resultaten van de TeamQ met elkaar te bespreken, kan er openheid ontstaan over de kwaliteit van samenwerken en samen opleiden. Daarbij is het belangrijk gezamenlijk de stap van meten naar verbeteren te maken en in te zien dat verbeteringen geen eenmalige acties zijn, maar een continue cyclisch kwaliteitsproces. Daarmee kunnen de opleiders werken aan een effectieve samenwerking binnen het opleidingsteam met arts-assistenten en met andere disciplines in multi-specialistische teams. De formele opleider kan reflecteren op leiderschapskwaliteiten aan de hand van de vier profielen en deze ontwikkelen in teamverband samen met de opleiders. Daarmee kan het samenwerkend leren gestalte krijgen. Vanuit die verworven collectiviteit zal een opleidingsteam beter kunnen anticiperen op externe ontwikkelingen en daarmee optimaal de dokter van de toekomst opleiden. 


\section{Dankwoord}

Een proefschrift over samenwerken schrijf je niet alleen. Vanaf het allereerste begin heb ik op de schouders van reuzen gestaan. Ik kan in dankbaarheid en met veel plezier terugdenken aan al die mensen die tijdens dit promotieonderzoek om mij heen stonden. Ik hoop met jullie allen nog veel samen te zijn, samen te doen en samen te onderzoeken.

Kiki Lombarts: wij vonden elkaar al zo'n 20 jaar geleden in onze liefde en ambitie voor de kwaliteit van de medische beroepsuitoefening. Toen jij wist dat ik onderzoek wilde doen, legde jij de verbinding met Albert en Cees: de 'mannen van Maastricht'. Ik zal dat nooit vergeten. Je trouw, je grondige feedback, je wijsheid en creatieve ideeën hebben mij gesteund en mede gevormd.

Albert Scherpbier en Cees van der Vleuten: al onze ontmoetingen staan me nog bij. Jullie ervaring en wijsheid stonden steeds naast al het plezier, het verdriet en de successen die jullie met mij deelden. Jullie onderlinge taakverdeling werkte subliem. De rol van boodschapper van het slechte nieuws en die van trooster en supporter, wisselden jullie af. Ik heb het allemaal zeer gewaardeerd.

Kiki, Albert en Cees: in al die jaren zijn onze gesprekken mij dierbaar geworden. Wat heb ik ongelofelijk veel van jullie geleerd. Dank voor jullie geduld en aandacht. Blind kon ik erop vertrouwen dat jullie mij oprechte feedback zouden geven en hoe blij waren jullie niet als ik die feedback ook werkelijk begreep! Door dit alles was ik in staat om door te gaan en is dit proefschrift er gekomen.

Leden van de opleidingsteams en arts-assistenten: onze ontmoetingen gingen over jullie praktijk, jullie professionaliteit en jullie opdracht in onderzoek, onderwijs \& opleiden en patiëntenzorg. Ik zat nooit om participanten verlegen en was steeds geraakt door jullie verhalen. Hierdoor is dit proefschrift relevanter geworden.

Renée van der Leeuw en Renée Stalmeijer: paranimfen kies je niet voor even, maar voor het leven. Jullie staan al heel wat jaren in lief en leed om mij heen en dat maakte onderzoek doen zo leuk. In allerlei kroegen hebben we onderzoeksvoorstellen, 
paradigma's en de methodologie bediscussieerd en bij vele gelegenheden is er ook vooral niet over onderzoek gesproken. Veel dank dat jullie ook tijdens de promotie echt naast me staan. Door jullie is het proefschrift rijker geworden.

Hoogleraren en collega onderzoekers medisch onderwijs uit AMC, MUMC, SLAZ, Diergeneeskunde, UMCG, VUmc en andere universitair medische centra: intens genoten heb ik van onze gesprekken, onze uitstapjes en ons samenwerken om met elkaar stik-goede onderzoekers te worden en te blijven. Ik leer door afkijken en dat kon ik bij jullie. Door jullie is het proefschrift veelkleurig geworden

Maas Jan Heineman: jij staat voor mij voor het AMC; de organisatie waar ik welkom was en waar ik me heel snel verbonden mee voelde. Met plezier denk ik aan onze gesprekken in de zoektocht naar wat de medische specialisten gaat helpen in de kwaliteit van zorg en opleiden. Dank voor je creatieve vragen, de belangstelling en de steun. Jij hebt dit proefschrift mede mogelijk gemaakt.

Onderzoekers van de Professional Performance Onderzoeksgroep: dank voor jullie onvoorwaardelijke en niet aflatende steun en support. Met humor, begrip, ontspanning, scherpe debatten en feedback, ieder vanuit zijn/haar eigen deskundigheid, was er contact en kon ik gewoon de 'drama-queen' zijn die leerde onderzoek te doen. Dit geldt ook voor de hoogleraar en de collega's van Center of Evidence Based Education in het AMC. Door jullie allen is dit proefschrift beter geworden.

De secretaresses in Maastricht: jullie hebben mij met raad en daad, humor en relativering bijgestaan. Ik kon altijd een beroep op jullie doen en dat vond ik nooit vanzelfsprekend. Veel dank daarvoor.

Intervisiemaatjes van de Leergang Opleidingskunde: jullie blijven mij herinneren aan de liefde voor leren, de 'wederzijdse aantrekkelijkheid' en de 'waarderende benadering'. Door jullie kon ik het leren aan en werd 'de plek der moeite' draaglijk. 
Mijn familie: jullie stonden in lief en leed dicht om mij heen en successen vierden jullie mee. Onze hechte band maakt dat ik steeds herinnerd wordt aan wat van waarde is en dat er voor alles een tijd is. Door jullie kreeg dit proefschrift een gouden randje.

Vrienden van de Leidse Schola Cantorum: door wekelijks te zingen, de regelmatige gesprekken, de (wandel)uitstapjes en de engelen-etentjes, kreeg ik de kans óf om te ontspannen óf om het er juist over te hebben. Vaak kreeg ik hierdoor weer zicht op de volgende stap. Om leven dat doorgaat! Jullie hebben het schrijven van dit proefschrift lichter gemaakt.

Roeimaatjes 'Mind in boat': als er ergens wordt samengewerkt, is dat in een veteranen vrouwenteam en als er ergens effectief en creatief wordt vergaderd, is dat in de vrouwenkleedkamer van de roeivereniging. We zitten al bijna tien jaar twee keer per week samen in de boot en zonder jullie niet aflatende belangstelling en energie was het proefschrift er vast niet gekomen.

leder die ik niet met naam en toenaam noem: oneindig veel dank voor jullie grote en kleine bijdrage aan de schoonheid van dit proefschrift.

Irin, Sarah en Hannah: een leven lang leren zit in mijn en dus ook in jullie bloed. Intens hebben jullie meegeleefd, van mijn afspraken met Kiki en de mannen tot de publicaties en afwijzingen. Ik heb bewondering voor de manier waarop jullie ieder op je eigen wijze het leven vorm geven. Het staat bol van het leren en inspireren. Door jullie word ik steeds herinnerd aan wat voor mij van waarde is: je kunt altijd opnieuw beginnen. Vooral samen! Aan jullie draag ik dit boekje op. 
146 | Chapter 7 


\section{Curriculum Vitae}

Irene Arida Slootweg was born on May $5^{\text {th }} 1957$ in Haarlemmermeer, The Netherlands. For over 35 years Irene has worked in within the Healthcare and Professional Education fields in the setting of the University Medical Centers in the Netherlands. Her experience is based on providing multidisciplinary care for critical patients in intensive care units. The overarching theme in both her work and research has been working and learning together with clinical teachers. Her ambition is to work with the medical professionals based on evidence based education. After completing her PhD 'Teamwork of Clinical Teachers in Postgraduate Medical Training' she continues to do research as a senior researcher within the Professional Performance Research group (www.professionalperformanceonline.com) and continues her job in training and advising faculty development programs. She also will remains employed by the Amsterdam Medical Center to develop policy and practice of quality improvement of postgraduate medical training.

\section{Work Experiences:}

- 2009 - present: PhD-student (part-time). Faculty of Health, Medicine and Life Sciences University. Maastricht.

- 2011-present: Trainer, coach and innovator (part-time); Teach the Clinical Teacher program, Academic Medical Center. Amsterdam.

- 2007 - 2011: Trainer Teach the Clinical Teacher; Free University Medical Center. Amsterdam.

- 2005 - 2011: Senior educationalist; Medical Faculty, Free University Medical Center. Amsterdam.

- 2000 - 2005: Senior innovator; Department Human Resource, Free University Medical Center. Amsterdam.

- 1992 - 2000: Senior innovator; Nursing faculty, Free University Medical Center. Amsterdam.

- 1988 - 1992: Quality Employee; Department Surgery, Leiden University Medical Center. Leiden.

- 1980 - 1987: IC- nurse; Intensive Care Surgical Unit, Leiden University Medical Center. Leiden. 


\section{Education:}

- Advanced Qualitative Research Course, School of Health Professions Education (SHE) Maastricht. 2013

- Intensive English Program. Radboud in'to Languages University Nijmegen. 2010

- Master course Qualitative research and case studies. Erasmus Universiteit Rotterdam. 2009

- Two-years course Learning Science, Stichting Opleidingskunde (Kessel-Smit) Utrecht. June 2006

- Bachelor Innovation in Health Care, Hogeschool Midden Nederland te Leusden. June 1989

- Two years course Intensive care in nursing. Academisch ziekenhuis te Leiden. July 1982

- $\quad$ Bacherlor Nursing, Diaconessenhuis, Leiden. February 1979

Publications:

- Slootweg IA, Lombarts MJMH, van der Vleuten CPM, Mann K, Jacobs J, Scherpbier AJJA. (2013) Clinical teachers' views on how teaching teams deliver and manage residency training. Medical Teacher. 35, 46-52.

- Bergman E., de Feijter J, Frambach J, Godefrooij M, Slootweg IA, Stalmeijer R, van der Zwet J. (2012): AM last page: A guide to research paradigms relevant to medical education. Academic Medicine 87.4 545.

- Van der Leeuw RM, Slootweg IA, Heineman MJ, Lombarts MJMH.(2013) Explaining how faculty act upon residents' feedback to improve their teaching performance. Medical Education 47.11 1089-98.

- $\quad$ Van der Leeuw RM, Slootweg IA. Twelve tips for making the best use of feedback. (2013) Medical Teacher 355 348-51.

- Slootweg IA, van der Vleuten CPM, Heineman MJ, Scherpbier AJJA, Lombarts MJMH. (2014) Program directors in their Role as Leaders of Teaching Teams in Residency Training. Medical Teacher Dec; 36 (12):1073-9.

- Slootweg IA, Lombarts MJMH, Boerebach BCM, Scherpbier AJJA, van der Vleuten CPM (2014). Development and Validation of an Instrument for Measuring the Quality of Teamwork in Teaching Teams (Team Q). PLOS one. 9(11);e112805. 
- Slootweg IA, Scherpbier AJJA, Leeuw van der RM, Heineman MJ, Vleuten van der CPM, Lombarts MJMH Speaking Up of Clinical Teachers in a Formal Meeting of Post Graduate Medical Training. (Submitted Advances in Health Sciences Education 2015)

Presentations:

Slootweg IA, Lombarts MJMH, Boerebach B, Heineman MJ, Scherpbier AJJA, van der Vleuten CPM.(2012). Criteria for teamwork in teaching teams in postgraduate medical training: A Delphi-study. Short communication presented at the Association for Medical Education in Europe (AMEE), Lyon, Frankrijk.

Slootweg IA, Lombarts MJMH, Boerebach B, Heineman MJ, Scherpbier AJJA, van der Vleuten CPM. (2013). Samenwerking in opleidersgroepen: Validatie van het TeamQ instrument. Research paper presented Congres Nederlandse Vereniging Medisch Onderwijs (NVMO conference), Egmond aan Zee, Netherlands.

Slootweg IA, Lombarts MJMH, Boerebach B, Heineman MJ, Scherpbier AJJA, van der Vleuten CPM. (2014). Development and Validation TeamQ, an instrument for evaluating Teamwork in Postgraduate Medical Training. Research paper presented at Association for Medical Education in Europe (AMEE), Milan. Italy.

Slootweg IA, van der Vleuten CPM, Heineman MJ, Scherpbier AJJA, Lombarts MJMH. (2014). Leiderschap van de formele opleider. Research paper presented Congres Nederlandse Vereniging Medisch Onderwijs (NVMO conference), Egmond aan Zee, Netherlands.

Slootweg IA. Scheele F, Baane C, Scherpbier AJJA, van der Vleuten CPM, Lombarts MJMH. (2014). Workshop: "Samenwerken binnen de opleidersgroep....Spelenderwijs uw probleem oplossen". Congres Nederlandse Vereniging Medisch Onderwijs (NVMO conference), Egmond aan Zee, Netherlands. 
Slootweg IA, Poolman R, Scherpbier AJJA, van der Vleuten CPM, Lombarts MJMH. (2014) Workshop: Leidinggeven aan opleidersgroepen. Congres Voortdurend Bewegen, Modernisering Medische Vervolgopleiding, Utrecht, Netherlands. 
